\title{
PREDICTION-GUIDED PERFORMANCE-ENERGY TRADE-OFF WITH CONTINUOUS RUN-TIME ADAPTATION
}

\author{
A Thesis \\ Presented to the Faculty of the Graduate School \\ of Cornell University \\ in Partial Fulfillment of the Requirements for the Degree of \\ Master of Science
}

by

Taejoon Song

May 2016 
(C) 2016 Taejoon Song

ALL RIGHTS RESERVED 


\begin{abstract}
Recent work has demonstrated that prediction-guided DVFS control can significantly improve the energy efficiency of interactive applications with little to no impact on user experience when running in isolation. In this thesis, I propose to add an on-line learning capability to the execution-time predictor, which enables the predictor to automatically adapt to changes in the environment such as interference from other applications and be easily applied across diverse platforms. This thesis introduces several techniques to address the overhead of performing on-line learning, including incremental training based on QR decomposition and explicit change detection for fast adaptation. In addition to the DVFS control, I show that the proposed prediction model can be used to intelligently select a core in a heterogeneous system. Experimental results on the ARM big.LITTLE platform show that the DVFS controller and core scheduler can effectively remove deadline misses even under significant interference from competing processes while consuming significantly lower energy compared to traditional schemes.
\end{abstract}




\section{BIOGRAPHICAL SKETCH}

Taejoon Song received his Bacheolor of Science degree in Semiconductor Systems Engineering from Sung Kyun Kwan University (SKKU) in 2013. After graduation, he worked at Samsung Electronics for one year, and joined the department of Electrial and Computer Engineering at Cornell University in 2014. His research interest lies at the intersection of computer architecture and realtime systems. 
This document is dedicated to all Cornell graduate students. 


\section{ACKNOWLEDGEMENTS}

I would like to express the deepest appreciation to my advisor, Prof. Edward Suh for his guidance, collaboration, and support. Without his supervision and constant help, this thesis would not have been possible. I also would like to thank my commitee member, Prof. Christopher Batten for his feedback and advice. He was also one of the best instructors who taught me Computer Architecture.

The thesis work is largely motivated by the previous work. Special thanks to Daniel Lo for his collaboration on our previous work. Also, special thanks to Tao Chen. Whenever I lost my reserch directions, he gave me a constant feedback with a great discussion. In addition, I thank everyone in the Suh Research Group and CSL.

Finally, I must express my very profound gratitude to my parents for provid-

ing me with unfailing support and continuous encouragement throughout my years of study and through the process of researching and writing this thesis. This accomplishment would not have been possible without them. Thank you. 


\section{TABLE OF CONTENTS}

Biographical Sketch . . . . . . . . . . . . . . . iii

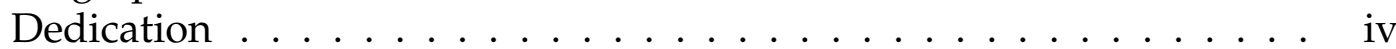

Acknowledgements . . . . . . . . . . . . . . . . . . . . . . .

Table of Contents . . . . . . . . . . . . . . . . . . . vi

List of Tables . . . . . . . . . . . . . . . . . . . . . . . . . ix

List of Figures ................... . . .

\begin{tabular}{lll}
\hline Introduction & 1
\end{tabular}

2 Background and Motivation $\quad 5$

2.1 Tasks, Jobs, and Deadlines . . . . . . . . . . . . . . . . 5

2.2 Execution Time Variation . . . . . . . . . . . . . . . . . 5

2.3 Previous DVFS Controllers . . . . . . . . . . . . . . 7

3 Prediction-Guided DVFS Controller 10

3.1 Prediction-Based Control . . . . . . . . . . . . . . . . . . . 10

3.2 Program Feature Generation . . . . . . . . . . . . . . . . . . . . . . . . . . . . . 11

3.3 Execution Time Prediction Model . . . . . . . . . . . . . . . . . . 15

3.4 DVFS Model . . . . . . . . . . . . . . . . . . . . . . 17

3.5 System-Level Framework . . . . . . . . . . . . . . . . . . . . . . . . . . . . . 20

3.5.1 Programmer Annotation . . . . . . . . . . . . . . . . . . 20

3.5 .2 Off-line Analysis $\ldots \ldots . . \ldots . . \ldots . . \ldots 21$

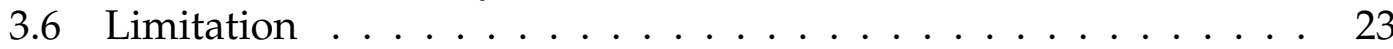

4 Adaptive Prediction Framework 24

4.1 Framework Overview . . . . . . . . . . . . . . . . . 25

4.2 Execution Time Prediction Model . . . . . . . . . . . . . . 26

4.3 On-line Model Training. . . . . . . . . . . . . . . . 28

4.3.1 Fast Updates Using QR Decomposition . . . . . . . . . . . . 29

4.3 .2 Conservative Prediction . . . . . . . . . . . . . . . 31

4.3 .3 Initial Training Phase . . . . . . . . . . . . . . 33

4.3.4 Fast Adaptation with Explicit Event Detection . . . . . . . 34

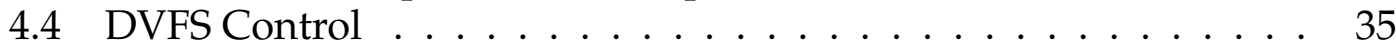

4.5 Task Migration on Heterogeneous Cores . . . . . . . . . . . . . . . 37

$\begin{array}{lll}5 & \text { Evaluation } & 39\end{array}$

5.1 Experimental Setup . . . . . . . . . . . . . . . . . . . . . . . . . . . . .

5.2 DVFS Controllers . . . . . . . . . . . . . . . . . . . . . . 40

5.3 Energy Savings and Deadline Misses . . . . . . . . . . . . . . . . . . . 41

5.3 .1 Without Interference $\ldots \ldots . \ldots . \ldots 42$

5.3 .2 With Interference . . . . . . . . . . . . . . . . . . . . . . . . . . . . . . 43

5.4 Heterogeneous Cores . . . . . . . . . . . . . . . . . . 45

5.5 Prediction Accuracy 
5.5 .1 Without Interference $\ldots \ldots \ldots$. . . . . . . . . . . . . . 47

5.5 .2 With Interference $\ldots \ldots \ldots$

5.5 .3 Multiple Platforms . . . . . . . . . . . . . . . . . . . . . . . 49

5.6 Analysis of Run-time Overheads . . . . . . . . . . . . . . . 50

5.7 On-line Training Sensitivity Studies . . . . . . . . . . . . . 51

$5.7 .1 \quad$ Accuracy vs. Number of Samples . . . . . . . . . . . . 51

5.7 .2 Impact of Interference Intensity . . . . . . . . . . . . . 52

5.7 .3 Under-prediction Trade-off . . . . . . . . . . . . . . . . 53

5.8 Idle Power $\ldots \ldots \ldots \ldots \ldots \ldots \ldots \ldots \ldots$

6 Related Work 56

6.1 Dynamic Voltage and Frequency Scaling . . . . . . . . . . . . 56

6.2 Execution Time Prediction $\ldots \ldots \ldots \ldots$. . . . . . . . . 57

6.3 Heterogeneous Core Management $\ldots \ldots \ldots \ldots$

7 Possible Extensions 59

7.1 Parallel Job Execution on Multi-core Systems . . . . . . . . . . . . 59

$7.1 .1 \quad$ Motivational Example $\ldots \ldots \ldots$. . . . . . . . . . . 60

$7.1 .2 \quad$ Assumption . . . . . . . . . . . . . . . . . . . 61

7.1 .3 Scheduling to Multiple Cores . . . . . . . . . . . . . . . 62

7.1 .4 Evaluation . . . . . . . . . . . . . . . . . . 64

7.1 .5 Limitation . . . . . . . . . . . . . . . . . . . . . 67

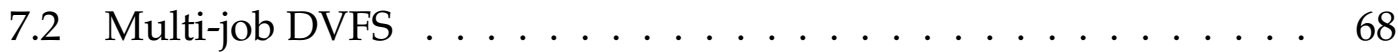

$7.2 .1 \quad$ Motivational Example . . . . . . . . . . . . . . . . . . 68

7.2 .2 Evaluation . . . . . . . . . . . . . . . . . . . . 70

7.2 .3 Limitation . . . . . . . . . . . . . . . . . . 72

7.3 Multi-threaded Applications . . . . . . . . . . . . . . . . . . 73

7.3.1 Execution Time Prediction of Multiple Threads . . . . . . . 73

7.3 .2 Evaluation . . . . . . . . . . . . . . . . . . 74

7.3 .3 Limitation . . . . . . . . . . . . . . . . . . . . 78

$\begin{array}{lll}8 \text { Conclusion } & 79\end{array}$

\begin{tabular}{|lr}
\hline A Solving Least Squares Approximation & $\mathbf{8 0}$
\end{tabular}

A.1 Linear Least Squares Approximation . . . . . . . . . . . . . . . . 80

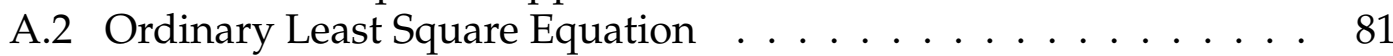

A.3 QR Decomposition $\ldots \ldots \ldots \ldots \ldots \ldots \ldots$. . . . . . . . 81

\begin{tabular}{|lll}
\hline Example of Fast Updates Using QR Decomposition & 84
\end{tabular}

B.1 Find the Model Coefficients . . . . . . . . . . . . . . . . . 84

B.1.1 Ordinary Least Square Equation . . . . . . . . . . . . . . 85

B.1.2 QR Decomposition . . . . . . . . . . . . . . . . . . . . 86

B.2 Update the Model with New Samples . . . . . . . . . . . . . . . 88

B.2.1 Ordinary Least Square Equation . . . . . . . . . . . . . . . 89 
B.2.2 QR Decomposition . . . . . . . . . . . . . . . . . . . 91

\begin{tabular}{lr}
\hline Bibliography & 94
\end{tabular} 


\section{LIST OF TABLES}

$3.1 \quad$ Variable and notation descriptions. $\ldots \ldots \ldots \ldots$

4.1 Variable and notation descriptions. $\ldots \ldots \ldots \ldots . \ldots .27$

5.1 Microarchitecture of Cortex-A15 and Cortex-A7 clusters. . . . . . 39

5.2 Benchmark descriptions and the number of features used. . . . . 40

A.1 Variable and notation descriptions. . . . . . . . . . . . 80 


\section{LIST OF FIGURES}

$2.1 \quad$ Example of tasks, jobs, and deadlines. . . . . . . . . . . . 5

2.2 Variation in execution time when interference happens in the middle. Each line represents running the exact same workload $($ sha), but with and without the interference. $\ldots \ldots \ldots 6$

$2.3 \quad$ Execution time of jobs for 2048 game [1] on two different platforms. 7

2.4 Actual execution time (red, circle) and predicted time by a PID controller (blue, diamond) for 2048 game. . . . . . . . . . . . 8

$3.1 \quad$ Overview of prediction-based control. . . . . . . . . . . . . 10

3.2 Example control flow graph. Each node is annotated with its number of instructions. . . . . . . . . . . . . . 12

$3.3 \quad$ Steps to predict appropriate frequency level from job input and

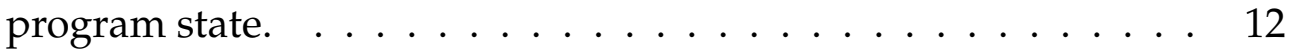

3.4 Example of feature counters inserted for conditionals, loops, and function calls. . . . . . . . . . . . . . . . . . . . . . . 13

$3.5 \quad$ Program slicing for control flow features. . . . . . . . . . . . . . 14

3.6 Average execution time of jobs (frames) for ldecode (video decod-

ing) as frequency level varies. $\ldots \ldots \ldots \ldots \ldots . \ldots \ldots$

3.7 The effective budget decreases due to slice and DVFS execution time. . . . . . . . . . . . . . . . . . 19

$3.8 \quad$ 95th-percentile switching times for DVFS. . . . . . . . . . . . 20

3.9 Example of programmer annotation to mark task boundaries and time budgets. $\ldots \ldots \ldots \ldots \ldots \ldots \ldots \ldots \ldots$

3.10 Overall flow for prediction-based DVFS control. . . . . . . . . . 21

$4.1 \quad$ Prediction-guided DVFS and task migration. . . . . . . . . . . . 24

4.2 Overall flow for the prediction-guided controller with continuous on-line learning. . . . . . . . . . . . . . . 26

4.3 The effective budget decreases due to the overhead of updating the model, the predictor, and DVFS switching. . . . . . . . 36

$5.1 \quad$ Normalized energy usage and deadline misses without interfer-

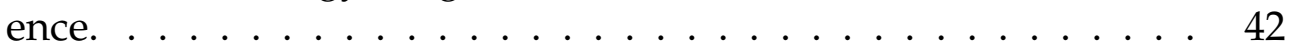

$5.2 \quad$ Normalized energy usage and deadline misses with interference. 44

5.3 Predicted and actual execution times for off-line (top) and online (bottom) training schemes with interference (sha). $\ldots . . .45$

$5.4 \quad$ Normalized energy usage, deadline misses, and big core usages with heterogeneous cores. . . . . . . . . . . . . . 46

5.5 Prediction errors for off-line and on-line training without inter-

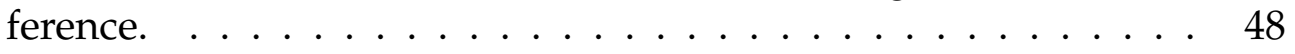

$5.6 \quad$ Prediction errors for off-line and on-line training during interference. .......................... 49

$5.7 \quad$ Prediction errors for on-line training on different processors. . . . 49 
5.8 Average overhead of the predictor, migration, DVFS switching, and updating the model. $\ldots \ldots \ldots \ldots$. . . . . . . . . . 50

5.9 Prediction errors as the number of samples for training is varied

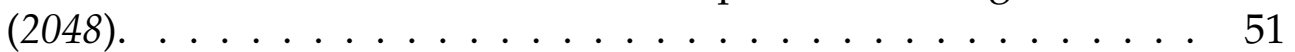

$5.10 \quad$ Normalized energy usage and deadline misses as the intensity level of interference is varied. . . . . . . . . . . . . . . . 52

5.11 Normalized energy usage and deadline misses as the underprediction penalty weight $(\alpha)$ varies. $\ldots \ldots \ldots \ldots \ldots \ldots$

5.12 Normalized energy usage for busy and idle cores (Normalized to maximum frequency of each case separately). . . . . . . . . . . 54

5.13 Normalized energy usage for busy and idle cores (Normalized to maximum frequency of busy). $\ldots \ldots \ldots \ldots \ldots$

$7.1 \quad$ Overview of prediction-based control. . . . . . . . . . . . . . . . 59

7.2 Motivational example ((a) no DVFS : This is run at maximum

frequency $f$, (b) DVFS with single core: This scales the frequency to just meet the deadline, and (c) DVFS with two cores : This not only scales the frequency, but also schedules the jobs to multiple

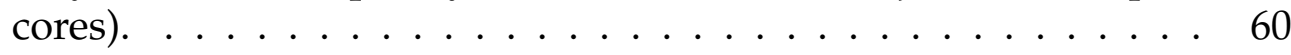

7.3 Overview of different scheduling algorithms. . . . . . . . . 63

7.4 Normalized energy usage and deadline misses with two A7 (lit-

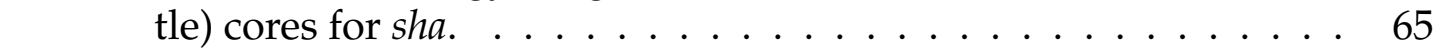

7.5 Normalized energy usage and deadline misses with two A15 (big) cores for sha. . . . . . . . . . . . . . . . 65

7.6 Motivational example ((a) no DVFS: This is run at maximum frequency $f$, (b) Single-job DVFS : This scales the frequency within a single job, and (c) Multi-job DVFS : This scales the frequency across multiple jobs). . . . . . . . . . . . . . . 68

7.7 Normalized energy usage and deadline misses with single-job and multi-job DVFS scheme. $\ldots \ldots \ldots \ldots$. . . . . . . . 70

\begin{tabular}{ll}
7.8 & Normalized energy usage and deadline misses as time budget is \\
\hline & varied (curseofwar). $\ldots \ldots \ldots \ldots \ldots \ldots \ldots \ldots \ldots \ldots \ldots \ldots \ldots$
\end{tabular}

7.9 Predicted time and actual time with one thread on one core (Water-Nsquared). . . . . . . . . . . . . . . . . . . . . . 75

7.10 Predicted time and actual time with four threads on different number of cores. From the top, the number of cores is 1, 2, 3, and 4, respectively (Water-Nsquared). . . . . . . . . . 76

7.11 Prediction errors as the number of threads and cores is varied (Water-Nsquared). . . . . . . . . . . . . . . . . . 77 
CHAPTER 1

\section{INTRODUCTION}

Many modern computing applications involve close interactions with the user where performance is measured by response time. In order to maintain a smooth user experience, responses must be provided in a timely manner within certain response-time requirements. Violations of these response-time requirements create a lag or stutter in the application that degrades the user experience. On the other hand, delivering responses significantly faster than the response-time requirements does not improve the user experience. For example, studies on human-computer interaction have shown that latencies of 100 milliseconds or better are required to maintain a good user experience [2, 3, 4], while response times faster than 50 milliseconds are imperceptible [5, 6]. For such interactive applications including gaming, web browsing, and video decoding, performance-energy trade-off techniques such as dynamic voltage and frequency scaling (DVFS) and heterogeneous architecture can be used to reduce energy usage while maintaining user experience.

For example, recent studies have shown that proactive, prediction-guided DVFS controllers [7, 8, 6, 9, 10] that predict the execution time of tasks based on program inputs can achieve significant energy savings while still meeting response-time requirements. The predictive DVFS approach consumes far less energy compared to traditional controllers such as the Linux power governors [11], which do not explicitly consider response-time requirements, and lead to much less response-time violations compared to reactive approaches [12, 13, 14, 15], which only use past history to determine future operating points. Naturally,

the effectiveness of such predictive DVFS approaches heavily depends on the 
accuracy and the costs of an execution-time predictor.

In this thesis, I propose a new execution-time prediction method and demonstrate its effectiveness through predictive DVFS control and migration on a heterogeneous multi-core platform. My approach adopts the profiling and program slicing techniques from a recently proposed DVFS controller [10] to automatically capture program features that determine execution time. The profiling and program slicing enable a prediction model to be quickly generated for a wide range of applications without significant efforts required for manually designed predictors [7, 8, 6, 9]. At the same time, I propose to use continuous on-line training to automatically adapt the model to operating environments at run-time.

The capability to automatically adapt to run-time changes addresses the main limitations of the previous prediction approach based on program slicing [10]. In the previous approach, a prediction model for each application is trained off-line for a specific platform and cannot change at run-time. As a result, the predictor performs poorly when there exists run-time variations such as interference from other applications. For example, the experimental results show that prediction-guided DVFS control with off-line training leads to response-time violation for over $35 \%$ of jobs when another application is also running together on the same core. The on-line training solves this problem by continuously updating the prediction model at run-time to incorporate the impact of interference. Experimental results show that the prediction-guided DVFS controller with run-time adaptation can meet response-time requirements even with unexpected run-time interference and still provide significant energy savings, close to $60 \%$ over the performance governor in Linux. 
The on-line training capability also enables a prediction model to be applied to a wide range of diverse platforms and configurations without the overhead of off-line training. In the previous prediction approach, a model needs to be trained off-line for each platform and configuration that may be used at runtime. This is a significant limitation in practice, especially for modern computing platforms with an increasing number of heterogeneous elements and runtime configurations such as cache partitioning. In this thesis, I demonstrate the flexibility of the new prediction model by using it to select an appropriate core on a heterogeneous multi-core with two types of cores. The experimental results show that the prediction-guided task migration can remove response-time violations while achieving the energy efficiency close to a small core by only using a big core when necessary. In the experiments, the prediction model for each core type was trained on demand at run-time without any off-line training.

The main challenge in performing on-line adaptation lies in managing the overhead of performing the continuous on-line model training. Unfortunately, I found that simply performing the existing off-line training algorithm at runtime added too much overhead to be practical. In this thesis, I solve this problem by introducing an incremental training method based on a QR decomposition technique where one new training sample can be added at a time with low overhead. I also found that simply training the model on-line is insufficient to effectively handle abrupt changes at run-time such as an introduction of interference from a new competing process. Because the on-line training slowly adapts the prediction model, an abrupt change can lead to poor prediction accuracy until the number of new samples after the change outweigh the number of old samples. In this work, I address the problem by explicitly detecting a significant change in the execution environment and re-train the model from scratch. 
The main contribution can be summarized as follows:

1. I present a new method for predicting execution time of an application, which captures both input-dependent variations and environmental variations at run-time such as program interference and diverse platforms. I use this predictor to create a prediction-guided DVFS controller that can automatically adapt to run-time variations.

2. I introduce techniques to reduce the overhead of run-time adaptation and improve the accuracy of execution-time prediction on an abrupt change in an environment.

3. I extend the DVFS controller to handle task migration in a heterogeneous system in order to further extend the performance-energy trade-off space.

The rest of the thesis is organized as follows. Chapter 2 introduces some background terminology and motivates the need for prediction-based DVFS control with continuous on-line learning. Chapter 3 introduces the previous work on the prediction-guided DVFS controller [10]. Chapter 4 discusses the proposed on-line learning technique and its use for selecting DVFS level and core type. Chapter 5 shows the evaluation results. Finally, Chapter 6 presents related work, and Chapter 7 and 8 conclude the thesis with a discussion of possible directions. 
CHAPTER 2

\section{BACKGROUND AND MOTIVATION}

\subsection{Tasks, Jobs, and Deadlines}

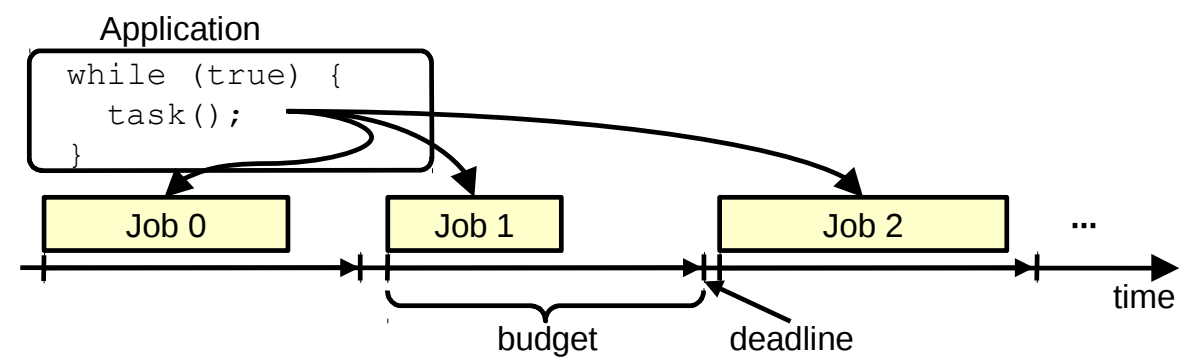

Figure 2.1: Example of tasks, jobs, and deadlines.

In this section, we define some terms which we will use in the rest of the thesis. First, we define a task as a portion of an application that has a responsetime requirement. For example, a video player application will consist of a task which must regularly output a frame (e.g., every 33 milliseconds for a 30 framesper-second video) in order for the video playback to be smooth. Each dynamic instance of the task is called a job. For a video player, the processing needed for each individual frame is a job. Each job has a deadline which is the point in time which they must complete by. The time period that they have to complete is referred to as the time budget. These terms are illustrated in Figure 2.1 .

\subsection{Execution Time Variation}

The execution time for a task can vary from job to job. If they can be predicted, these execution time variations provide an opportunity to save energy using 


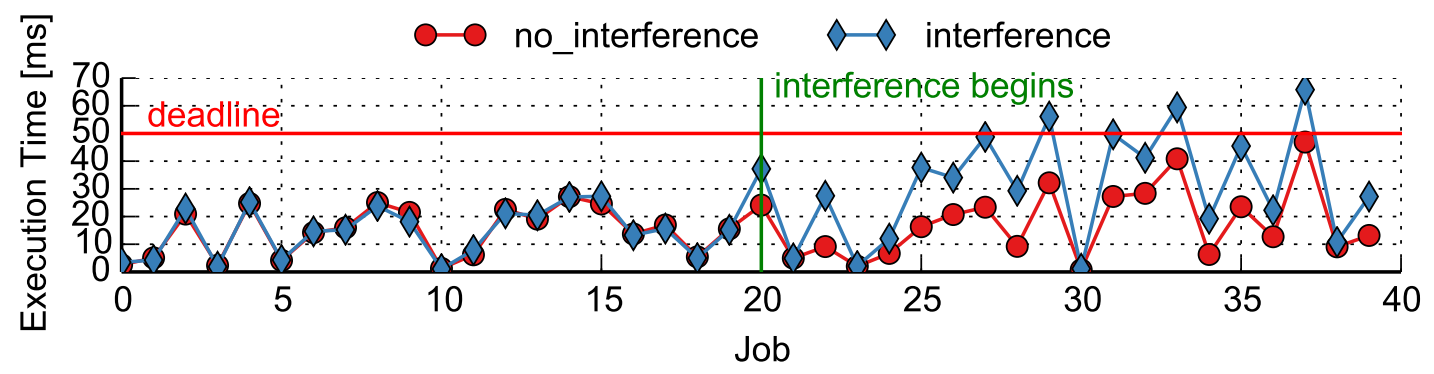

Figure 2.2: Variation in execution time when interference happens in the middle. Each line represents running the exact same workload (sha), but with and without the interference.

performance-energy trade-off techniques, without impacting user experience. For example, dynamic voltage and frequency scaling (DVFS) can be used to slow down a fast job in order to save energy. As long as the job still finishes by its deadline, then there will be no impact on user experience.

A task's execution time may vary from job to job due to multiple reasons. One major source of variation comes from changes in program inputs. For example, different frames in a video player often require a different amount of processing time. The previous work on prediction-guided DVFS [10] shows that such input-dependent variations can be predicted with a model based on a program's control flow features.

The execution time can also vary due to changes in a program's execution environment. For example, Figure 2.2 shows the execution times of each job for the SHA-1 hash function with and without interference. Here, the interference was added by running additional applications on the same core together with SHA-1, starting at Job 20. As shown in the figure, the execution time becomes significantly higher with the interference. If a prediction model does not capture the variation from run-time interference, a prediction-guided DVFS leads to poor user experiences due to deadline misses. 


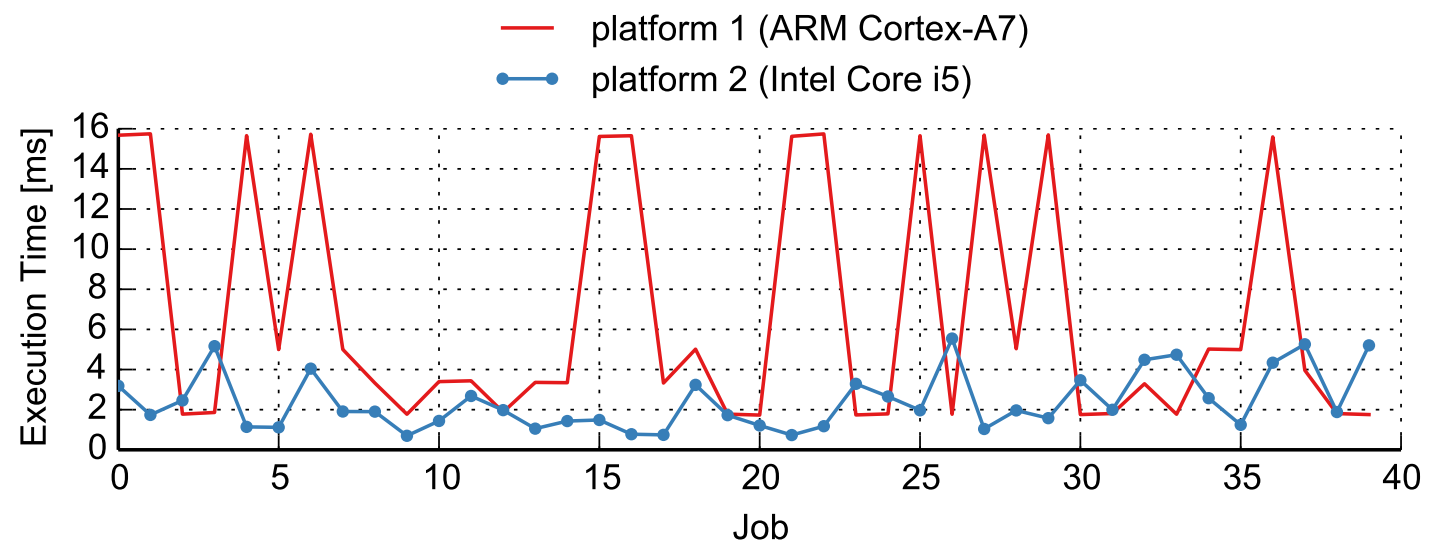

Figure 2.3: Execution time of jobs for 2048 game [1] on two different platforms.

In addition to the run-time interference, the execution time of a task also varies significantly across platforms. Figure 2.3 shows the difference in execution time of a game on two different platforms: ARM Cortex-A7 and Intel Core i5. An execution environment for a program can vary considerably even on a single processor because modern processors can run with many configurations. For example, a processor cache may be allocated differently using a partitioning mechanism. Similarly, memory controllers and on-chip interconnect networks may run with multiple clock frequencies or may include QoS mechanisms to control resource allocations. This diversity in platforms introduces another challenge that an execution time prediction model must handle in order to be broadly applicable.

\subsection{Previous DVFS Controllers}

Traditional DVFS controllers, such as the power governors found in Linux [11], do not take into account response-time requirements when selecting an operat- 


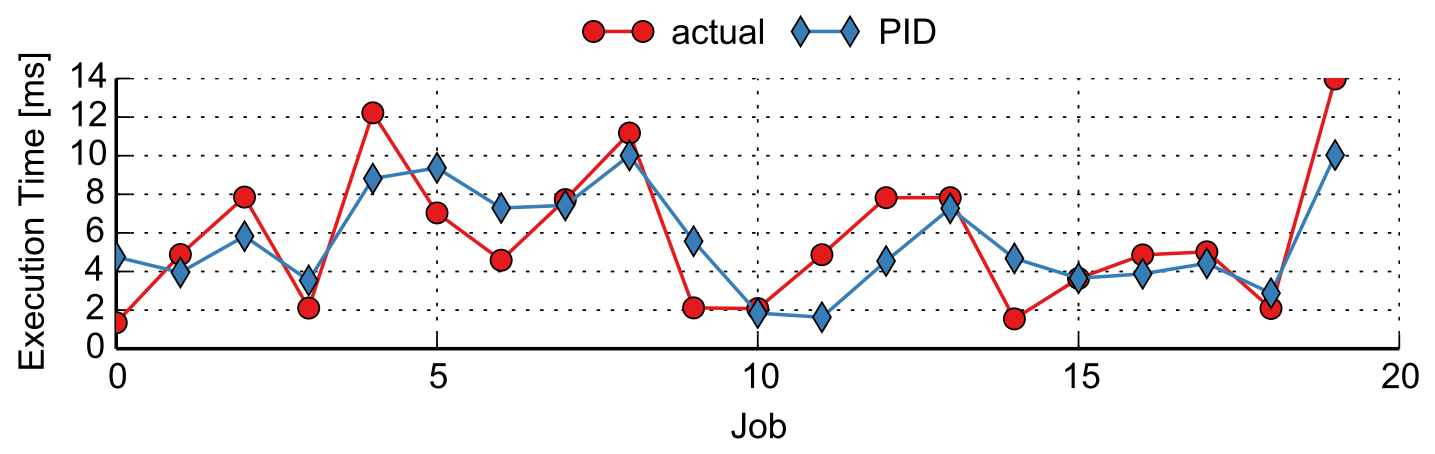

Figure 2.4: Actual execution time (red, circle) and predicted time by a PID controller (blue, diamond) for 2048 game.

ing point. A number of DVFS controllers have been designed that use the past history of job execution times to predict future execution times [12, 13, 14, 15]. These reactive approaches can capture slow changing variations in execution time, but fail to capture the fast job-to-job variations that can exist. For example, Figure 2.4 compares the actual execution time of jobs from the 2048 game with the predicted time used by a PID-based controller. As illustrated, the predicted time lags behind the actual execution time, leading to either wasted energy or missed deadlines.

More recently, Lo et al. [10] proposed a method for creating DVFS controllers that predict the execution times of jobs based on input values and program state in order to decide on the appropriate operating point. This method was shown to be quite effective in capturing input-dependent execution time variations using control flow features such as loop counts, branch directions, and others. In essence, this approach generates a prediction model, which can map input values to an execution time on a fixed platform with no interference. The model is built using off-line profiling and training using sample executions, and quickly evaluated at run-time using program slicing. In Chapter 3, we will discuss this work in more detail. 
Unfortunately, this prediction-guided DVFS method cannot adapt to runtime interference because its model is fixed. For the example in Figure 2.2, this DVFS controller will set an operating point based on the prediction similar to the line with no interference, and suffer from deadline misses when there is interference. Moreover, the prediction model is not easily portable to different platforms because expensive, off-line training is needed for each target platform and configuration. 


\section{CHAPTER 3}

\section{PREDICTION-GUIDED DVFS CONTROLLER}

Recent work [10] proposed the prediction-guided DVFS controllers that predict the execution times of jobs based on input values and program state to estimate the appropriate DVFS level. Since my approach extends this work and adopts several techniques from this work, I will discuss this work in this chapter. Note that the DVFS controller described in this chapter is mainly developed by Daniel Lo with my help in carrying out experiments. This work does not represent a new contribution of this thesis.

\subsection{Prediction-Based Control}

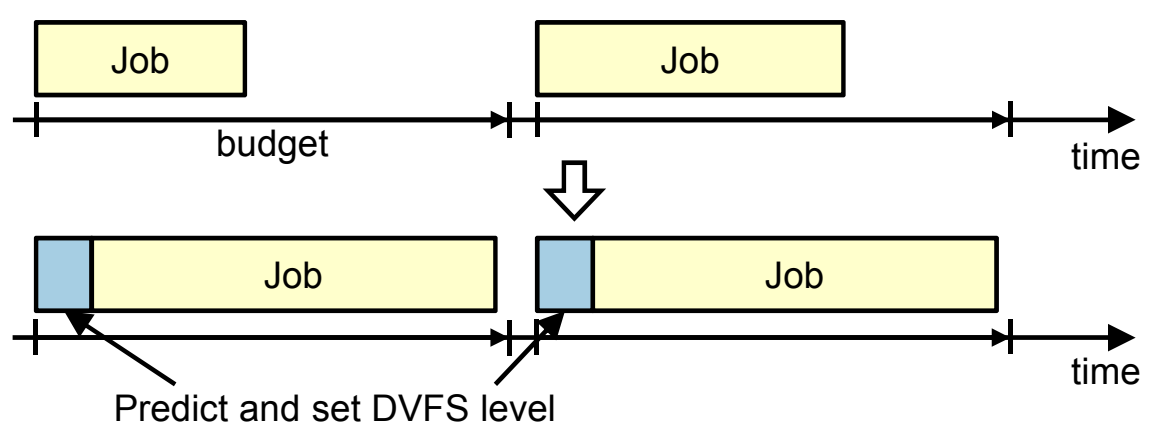

Figure 3.1: Overview of prediction-based control.

Our goal in this work is to develop a general and automated framework that will, given a task and its time budget requirement, create a prediction-based DVFS controller that can minimize energy usage without missing deadlines. Figure 3.1 shows an overview of the operation of our proposed prediction-based controller. The basic idea is to pre-pend tasks with a small segment of code. This segment of code will predict the appropriate DVFS level to use for each of the task's jobs depending on the job's input and current program state. 
The main source of execution time variation between jobs is due to different inputs and program state. Thus, the main challenge in creating a predictionbased DVFS controller is determining how to map job input and program state values to the appropriate DVFS frequency level. In general, finding a direct mapping from input values to frequency levels is challenging because the mapping can be irregular and complicated. In addition, this mapping varies from application to application. For example, for one application, pressing the "down" key may correspond to a large increase in execution time while for other applications it may have no effect on execution time. Our solution is to take advantage of the program source to give us hints about how input values and program state will affect execution time. We use the program source to automatically generate a prediction-based DVFS controller.

\subsection{Program Feature Generation}

The basic reasoning behind our prediction methodology is that, to first-order, execution time correlates with the number of instructions run. Variations in the number of instructions run are described by the control flow taken by a specific job. For example, consider the control flow graph for a task shown in Figure 3.2. Each node is marked with its number of instructions; (a) Taking left branch results in total $64(=1+10+3+10 \times 5)$ instructions, while $(b)$ taking right branch results in total $21(=1+2+3+3 \times 5)$ instructions. By knowing which branch is taken and the number of loop iterations, we can know the number of instructions executed and estimate the execution time. With an estimate of the execution time, we can then estimate the performance impact of DVFS and choose an appropriate frequency and voltage level to run at in order to just meet 


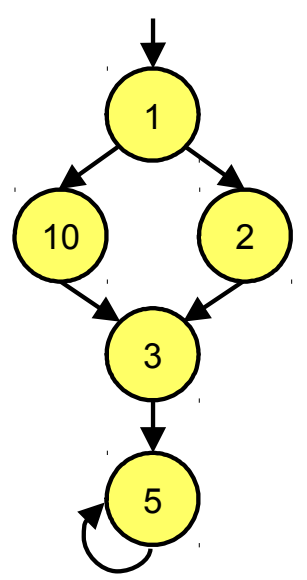

Original

Control

Flow (a)

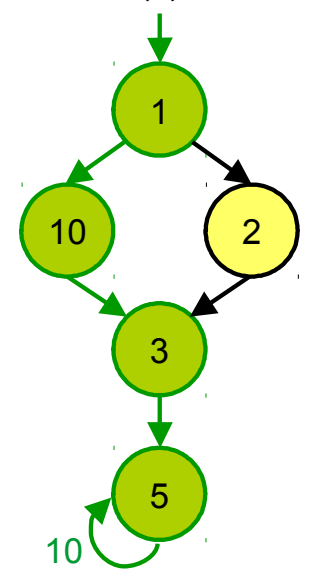

Left branch taken

\& 10 iterations, \# of inst is $1+10+3+10^{\star} 5$ (b)

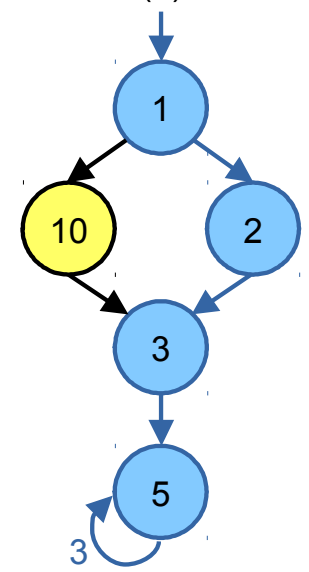

Right branch taken

\& 3 iterations, \# of inst is $1+2+3+3^{*} 5$

Figure 3.2: Example control flow graph. Each node is annotated with its number of instructions.

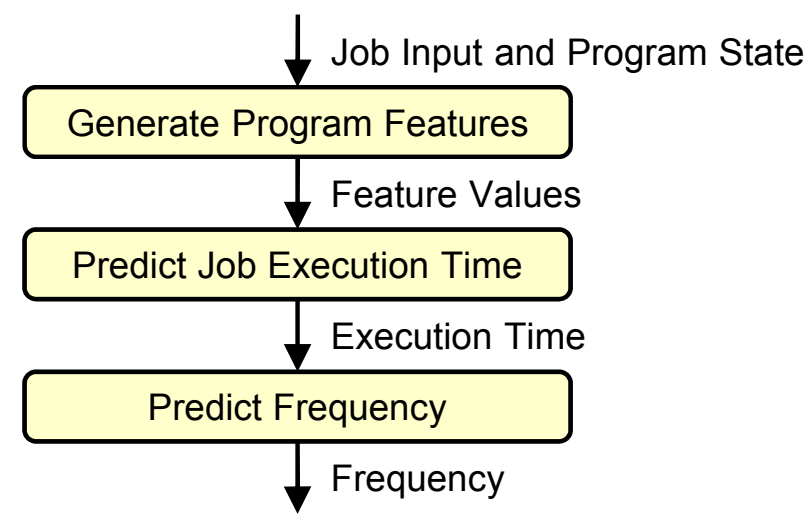

Figure 3.3: Steps to predict appropriate frequency level from job input and program state.

the deadline.

Figure 3.3 shows the main steps in this method. We first instrument the task source code and use program slicing to create a code fragment that will calculate control flow features for a job. The code fragment is run before a job executes in order to generate the control flow features (Section 3.2). Next, we use a linear 


\begin{tabular}{|c|c|c|}
\hline & Original Code & Instrumented Code \\
\hline 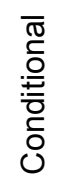 & $\begin{array}{l}\text { if (condition) }\{ \\
\} . .\end{array}$ & $\begin{array}{l}\text { if (condition) }\{ \\
\quad \text { feature }[\theta]++; \\
\quad \ldots\end{array}$ \\
\hline 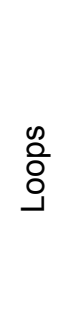 & $\begin{array}{l}\text { for }(i=0 ; i<n ; i++)\{ \\
\} \\
\text { while }(n=n->n e x t)\{ \\
\text { \}.. }\end{array}$ & $\begin{array}{l}\text { feature }[1]+=n ; \\
\text { for }(i=0 ; i<n ; i++)\{ \\
\quad \ldots \\
\} \\
\text { while }(n=n->n e x t)\{ \\
\quad \text { feature }[2]++; \\
\text { ‥ }\end{array}$ \\
\hline$\overline{\bar{\sigma}}$ & $($ *func $)()$; & $\begin{array}{l}(* \text { func })() ; \\
\text { feature }[3]=\text { func; }\end{array}$ \\
\hline
\end{tabular}

Figure 3.4: Example of feature counters inserted for conditionals, loops, and function calls.

model, which we train off-line, to map control flow features to an execution time estimate for the job (Section 3.3). Finally, we use classical linear models $[16,17]$ that describe the frequency-performance trade-off of DVFS to select an appropriate frequency (Section 3.4).

The first step needed for our prediction is to generate control flow features. That is, we want to know the control flow of a task when executing with a specific input and program state. For this purpose, we instrument the task source to count these control flow features. We instrument the task to count the following features:

- Number of times each conditional branch is taken

- Number of iterations for each loop

- Address of each function pointer call

Figure 3.4 shows examples of how these features are instrumented. We focus on control flow features because these explain most of the execution time variation. 


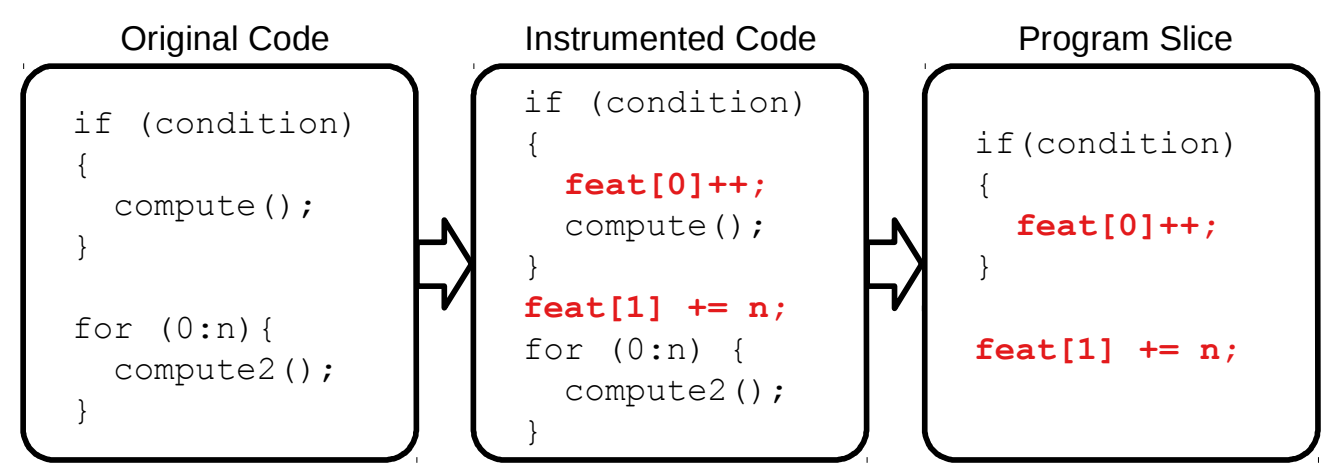

Figure 3.5: Program slicing for control flow features.

However, other features, such as variable values or memory accesses, could be included to improve the prediction accuracy.

Generating these features using an instrumented version of the task code is not suitable for prediction because the instrumented task will take at least as long as the original task to run. Instead, we need to quickly generate these features before the task execution. In order to minimize the prediction execution time, we use program slicing [18, 19] to produce the minimal code needed to calculate these features. Figure 3.5 shows a simple example of this flow. By removing the actual computation and only running through the control flow, the execution time can be greatly reduced. Since the information from this slice will ultimately be used to make a heuristic decision on DVFS control, the slice does not need to perfectly calculate the features. Instead, using an approximate slice can reduce the slice's size and execution time. For example, our tool tracks dependences based only on variable names and ignores possible pointer aliasing. As long as inaccuracies in the generated features are low, an approximate slice is adequate for our prediction needs. We refer to the resulting program slice that computes the control flow features as the prediction slice or simply as the slice.

One problem that arises with running this prediction slice before a task is the 


\begin{tabular}{|c|l|l|}
\hline Variable & Type & Description \\
\hline \hline $\bar{y}$ & Scalar & Predicted execution time \\
\hline $\mathbf{X}$ & Vector & Feature values \\
\hline $\boldsymbol{\beta}$ & Vector & Model coefficients \\
\hline \hline $\mathbf{y}$ & Vector & Profiled execution times \\
\hline $\mathbf{X}$ & Matrix & Profiled feature values \\
\hline $\mathbf{X} \boldsymbol{\beta}-\mathbf{y}$ & Vector & Prediction errors \\
\hline \hline$\alpha$ & Scalar & Under-predict penalty weight \\
\hline$\gamma$ & Scalar & Number of terms penalty weight \\
\hline$\|\cdot\|$ & Scalar & L2-norm (sum of squares) \\
\hline$\|\cdot\|_{1}$ & Scalar & L1-norm (sum of absolute values) \\
\hline
\end{tabular}

Table 3.1: Variable and notation descriptions.

issue of side-effects. That is, the slice could write to global variables and break the correctness of the program. In order to prevent this, the slice creates local copies of any global variables that are used. Values for these local copies are updated at the start of the slice and writes are only applied to the local copy. A similar process is applied to any arguments that are passed by reference.

\subsection{Execution Time Prediction Model}

Next, we need to predict the execution time from the control flow features. This section describes our model that maps features to execution time. Table 3.1 summarizes the variables and notation that are used in this section. We use a linear model to map features to execution time as this captures the basic correlation. Higher-order or non-polynomial models may provide better accuracy. However, a linear model has the advantage of being both simple to train and fast to evaluate at run-time. In addition, it is always convex which allows us to use convex optimization-based methods to fit the model. Our linear model can be 
expressed as

$$
\bar{y}=\mathbf{x} \boldsymbol{\beta}
$$

where $\bar{y}$ is the predicted execution time, $\mathbf{x}$ is a vector of feature values, and $\beta$ are the coefficients that map feature values to execution time. These $\beta$ coefficients are fit using profiling data. We profile the program to produce a set of training data consisting of execution times $\mathbf{y}$ and feature vectors $\mathbf{X}$ (i.e., each row of $\mathbf{X}$ is a vector of features, $\mathbf{x}_{i}$, for one job). Note that for addresses recorded for function calls, each unique address represents a different control flow which can correlate to a different effect on execution time. In order to properly represent each address as a feature, addresses recorded for function calls are converted to a one-hot encoding indicating whether particular function addresses were called or not.

The most common way to fit a linear model is to use least squares regression. Least squares regression finds the coefficients $\beta$ that minimize the mean square error:

$$
\min _{\boldsymbol{\beta}}\|\mathbf{X} \boldsymbol{\beta}-\mathbf{y}\|^{2}
$$

Essentially, this aims to minimize the sum of the absolute errors in the prediction. That is, it weighs negative and positive errors equally. However, these two errors lead to different behaviors on our system. Negative errors (underprediction) lead to deadline misses since we predict the job to run faster than its actual execution time. On the other hand, positive errors (over-prediction) result in an overly conservative frequency setting which does not save as much energy as possible. In order to maintain a good user experience, we would prefer to avoid deadline misses, possibly at the cost of energy usage. In other 
words, we should place greater weight on avoiding under-prediction as opposed to over-prediction.

We can place greater weight on under-prediction by modifying our optimization objective:

$$
\min _{\boldsymbol{\beta}}\|\operatorname{pos}(\mathbf{X} \boldsymbol{\beta}-\mathbf{y})\|^{2}+\alpha\|n e g(\mathbf{X} \boldsymbol{\beta}-\mathbf{y})\|^{2}
$$

where $\operatorname{pos}(x)=\max \{x, 0\}$ and $\operatorname{neg}(x)=\max \{-x, 0\}$ and these functions are applied element-wise to vectors. Thus, $\|\operatorname{pos}(\mathbf{X} \boldsymbol{\beta}-\mathbf{y})\|^{2}$ represents the over-prediction error and $\|n e g(\mathbf{X} \boldsymbol{\beta}-\mathbf{y})\|^{2}$ represents the under-prediction error. $\alpha$ is a weighting factor that allows us to place a greater penalty on under-predictions by setting $\alpha>1$. Since this objective is convex, we can use existing convex optimization solvers to solve for $\boldsymbol{\beta}$.

Coefficients which are zero imply that the corresponding control flow features do not need to be calculated by the prediction slice. We can use this information to further reduce the size and execution time of the prediction slice. We extend our optimization objective to favor using less features by using the Lasso method [20]:

$$
\min _{\boldsymbol{\beta}}\|\operatorname{pos}(\mathbf{X} \boldsymbol{\beta}-\mathbf{y})\|^{2}+\alpha\|n e g(\mathbf{X} \boldsymbol{\beta}-\mathbf{y})\|^{2}+\gamma\|\boldsymbol{\beta}\|_{1}
$$

where $\|\cdot\|_{1}$ is the L1-norm and $\gamma$ is a weighting factor that allows us to trade-off prediction accuracy with the number of features needed.

\subsection{DVFS Model}

Given a predicted execution time, we need to estimate how the execution time will change with varying frequency. For this, we use the classical linear model 


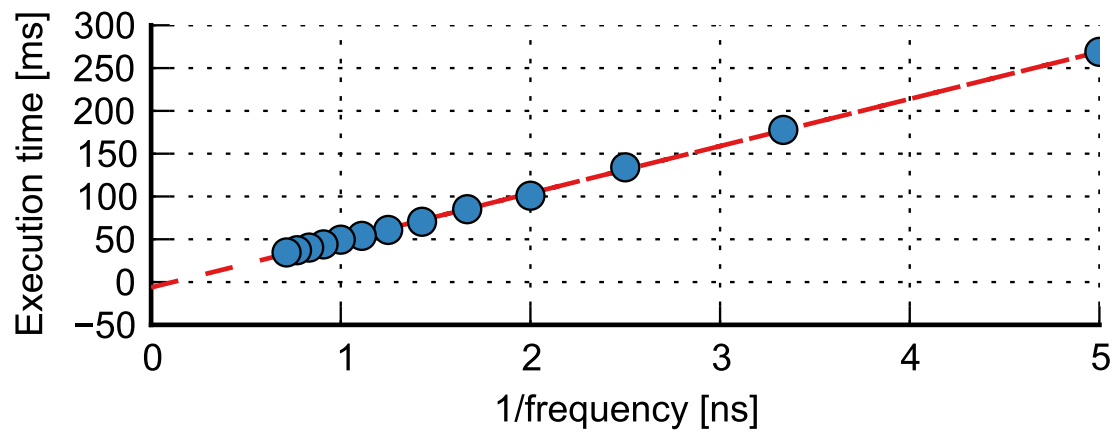

Figure 3.6: Average execution time of jobs (frames) for ldecode (video decoding) as frequency level varies.

found in literature [16, 17]:

$$
t=T_{\text {mem }}+N_{\text {dependent }} / f
$$

where $t$ is the execution time, $T_{m e m}$ is the memory-dependent execution time that does not scale with frequency, $N_{\text {dependent }}$ is the number of CPU cycles that do not overlap with memory and scale with frequency, and $f$ is the frequency. In order to verify this linearity assumption, we measured average job execution times as frequency was varied. Figure 3.6 shows the average job execution time versus $1 / f$ for ldecode (video decoder application). We can see that $t$ and $1 / f$ do show a linear relationship. We saw similar results for the other applications we tested.

By predicting the execution time at two points, we can determine $T_{m e m}$ and $N_{\text {dependent }}$ for a job and calculate the minimum frequency $f$ to satisfy a given time budget $t_{\text {budget }}$. More specifically, we predict the execution time $\bar{t}_{\text {fmin }}$ at minimum frequency $f_{\min }$ and the execution time $\bar{t}_{f \max }$ at maximum frequency $f_{\max }$. Using these two points, we can calculate $T_{m e m}$ and $N_{\text {dependent }}$ as

$$
\begin{aligned}
N_{\text {dependent }} & =\frac{f_{\min } f_{\max }\left(\bar{t}_{f \min }-\bar{t}_{f \max }\right)}{f_{\max }-f_{\min }} \\
T_{\text {mem }} & =\frac{f_{\max } \bar{t}_{\text {fmax }}-f_{\min } \bar{t}_{\text {fmin }}}{f_{\max }-f_{\min }}
\end{aligned}
$$




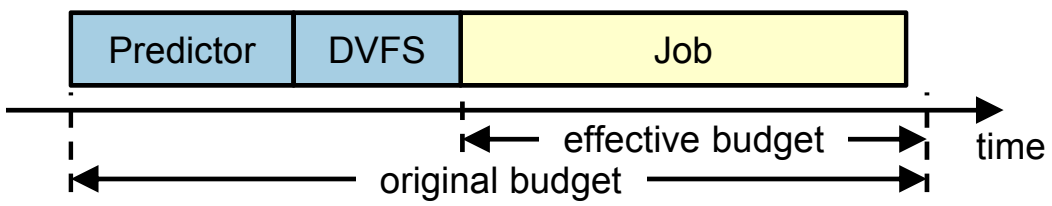

Figure 3.7: The effective budget decreases due to slice and DVFS execution time.

For a given budget $t_{\text {budget }}$, we want the minimum frequency $f_{\text {budget }}$ that will meet this time. This can be calculated as

$$
f_{\text {budget }}=\frac{N_{\text {dependent }}}{t_{\text {budget }}-T_{\text {mem }}}
$$

Since execution time can vary even with the same job inputs and program state, we add a margin to the predicted execution times used $\left(t_{f \min }\right.$ and $\left.t_{f \max }\right)$. In our experiments we used a margin of $10 \%$. A higher margin can decrease deadline misses while a lower margin can improve the energy savings. The resulting predicted frequency is the exact frequency that we expect will just satisfy the time budget. However, DVFS is only supported for a set of discrete frequency levels. Thus, the actual frequency we select is the smallest frequency allowed that is greater than $f_{\text {budget }}$.

The execution of the prediction slice and DVFS switch reduces the amount of time available for a job to execute and still satisfy its budget. Thus, the effective budget when choosing a frequency to run at needs to consider these overheads (see Figure 3.7). Although the execution time of the prediction slice can be measured, the DVFS switching time must be estimated, as the switch has not been performed yet. This is done by microbenchmarking the DVFS switching time. Figure 3.8 shows the 95th-percentile DVFS switching times for our test platform [21] for each possible start and ending frequency. We use the 95thpercentile switching times (i.e., the 5 th highest time out of our 100 runs) in order 


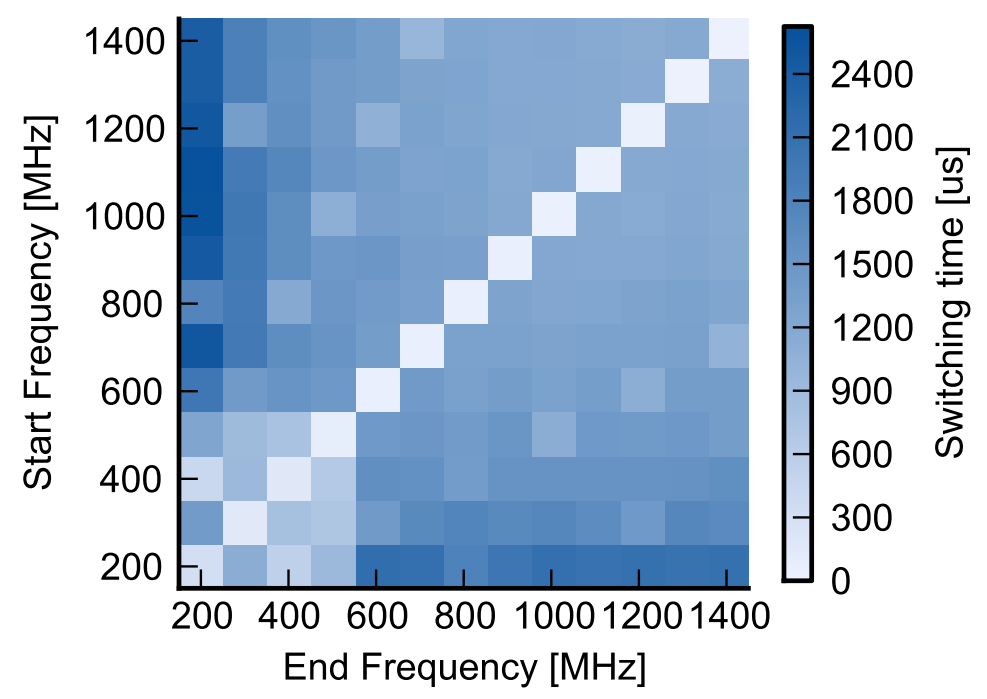

Figure 3.8: 95th-percentile switching times for DVFS.

to be conservative in our estimate of DVFS switching time while omitting rare outliers.

\subsection{System-Level Framework}

This section describes the overall framework and operation of our predictionbased controller.

\subsubsection{Programmer Annotation}

In order to apply our framework to an application, we require the individual tasks and their time budgets to be identified. These are identified by programmer annotations. The programmer must annotate the start and the end of a task and the desired response-time requirement. Figure 3.9 shows an example of this annotation. For ease of analysis and to ensure that tasks that start always 


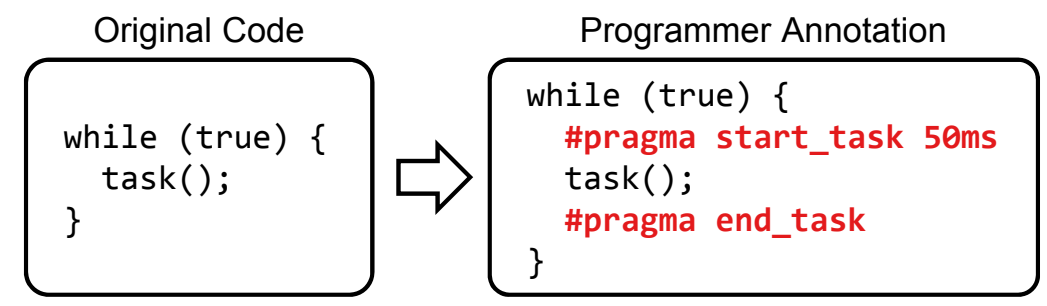

Figure 3.9: Example of programmer annotation to mark task boundaries and time budgets.

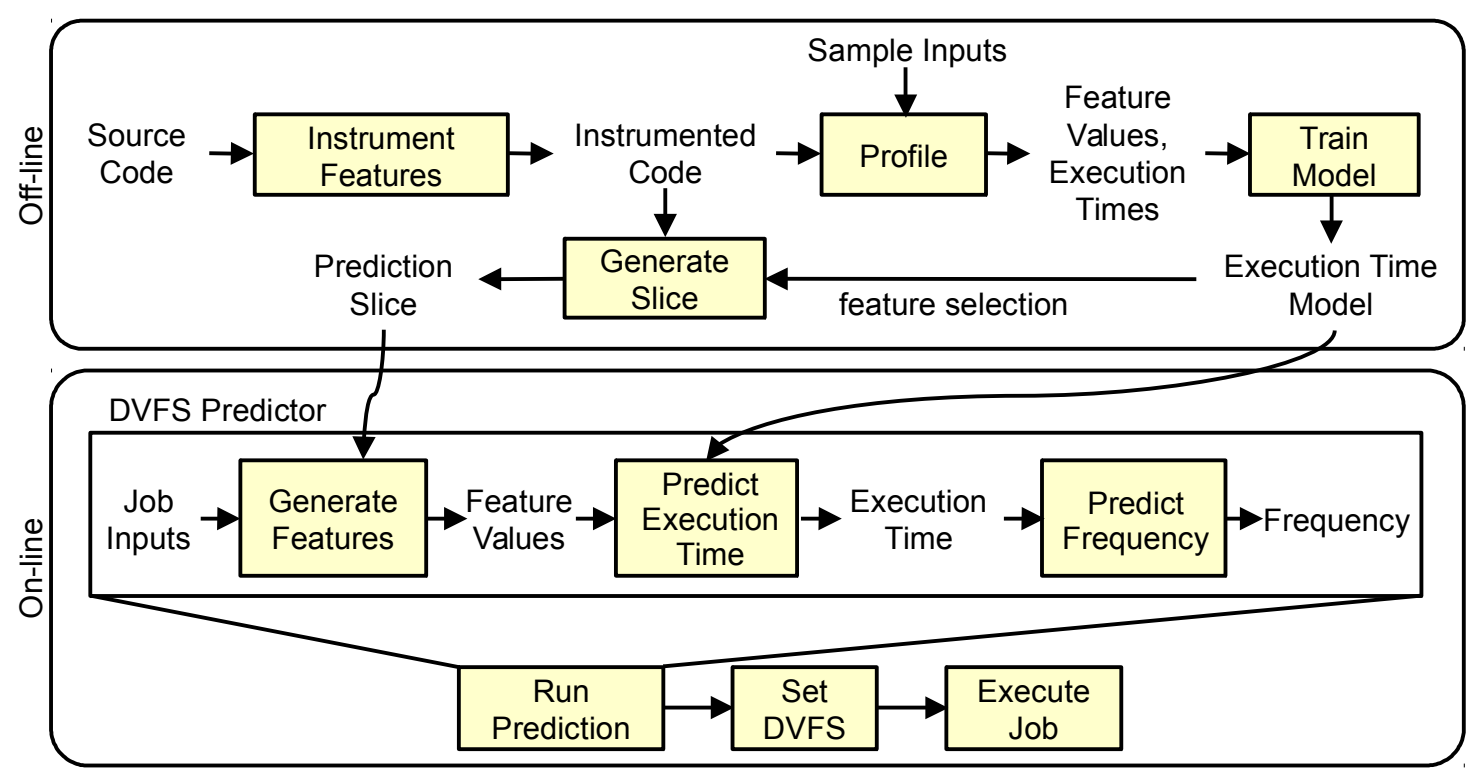

Figure 3.10: Overall flow for prediction-based DVFS control.

end, we require the start and end of a task to be within one function. Arbitrary code paths can be modified to fit this model by using a wrapper function or rewriting the code. Multiple non-overlapping tasks can be supported, though we only considered one task in the applications we tested.

\subsubsection{Off-line Analysis}

Figure 3.10 shows the overall flow of our framework for creating predictionbased DVFS controllers. Given programmer annotation to identify tasks, we 
can automatically instrument these tasks to record control flow features. Offline, we profile these tasks in order to collect traces of feature values and job execution times. This is used to train our execution time prediction model, as described in Section 4.2. Since execution time depends on the specific hardware and platform that an application is run on, profiling and model training needs to be done for the platform that the application will be run on. For common platforms, the program developer can perform this profiling and distribute the trained model coefficients with the program. Alternatively, profiling can be done by the user during application installation.

The trained execution time model only requires a subset of all the features to perform prediction. Specifically, features whose model coefficients are zero can be excluded from the prediction slice. Program slicing is used to create a minimal code fragment to calculate only the needed control flow features. Note that since the features needed depends on the training of the execution time prediction model, which is platform-dependent, the features needed could vary across platforms. However, we expect the features that are needed are primarily a function of the task semantics (i.e., execution time variations across control paths) rather than the platform it is run on. In fact, we compared the predictions made for an x86-based (Intel Core i7) platform when using the features selected for an ARM-based ODROID-XU3 [21] platform and for the features selected for the x86 platform itself. For all but three of the benchmarks we tested, the features selected were exactly the same. For one of these three benchmarks, the features selected by the x 86 platform were a subset of those selected by the ARM platform and so the predicted times were exactly the same. For the remaining two benchmarks, the predicted times differed by less than 3\%. Although we had to re-train the execution time model coefficients, the same prediction slice 
was applicable across both platforms.

\subsection{Limitation}

In this work, we presented a framework for prediction-based DVFS control. This controller predicts the appropriate frequency to use for a job in order to minimize energy and just meet the required deadline. Our prediction works by first generating control flow features, then predicting the execution time of the job, and finally predicting the appropriate frequency to use. Our results show $56 \%$ average energy savings over running at maximum frequency with almost no deadlines misses.

Unfortunately, there are some limitations to this work. Since a prediction model is fixed after it is trained off-line for a specific platform, the model cannot capture execution environmental changes at run-time. As discussed in Section 2.2. for example, starting or terminating other applications may interfere the original program by competing the shared resources.

Also, a model needs to be trained off-line for different platforms and configurations, as we have shown in Figure 2.3 . This can be a significant limitation in practice, especially for modern computing platforms with an increasing number of heterogeneous elements and run-time configurations such as cache partitioning.

These limitations motivate my thesis work. In the next chapter, I will discuss how to overcome such limitations with the on-line learning capability. 


\section{CHAPTER 4}

\section{ADAPTIVE PREDICTION FRAMEWORK}

The goal in this thesis is to develop a prediction-guided control framework for performance-energy trade-off techniques in a way that can handle all three major sources of execution time variations discussed in Section 2.2. input-dependent variations, variations from run-time interference, and variations across different platforms and configurations. In particular, this work focuses on applying this adaptive prediction method to guide DVFS control and migration between heterogeneous cores.

Figure 4.1 illustrates how this prediction-guided DVFS and task migration work compared to just running jobs on a little core without any prediction. In the prediction-guided approach, my framework builds a model that can predict the execution time of each job. This model is used at the beginning of each job's time budget to estimate the DVFS levels and core types that can meet the deadline for the job. Then, the controller migrates a job and/or adjust the DVFS level in order to meet the deadline with minimal energy consumption. In the example, the controller adjusts a DVFS level on the little core before running

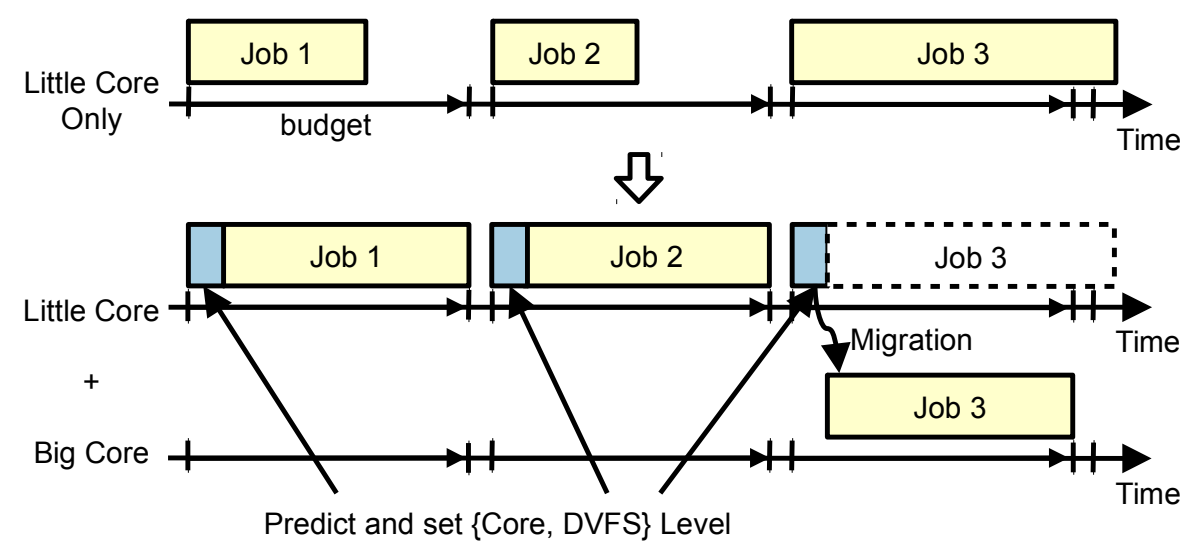

Figure 4.1: Prediction-guided DVFS and task migration. 
Job 1 and Job 2 based on the execution time prediction. For Job 3, the predictor determines that the deadline cannot be met on the little core and migrates the job to the big core.

\subsection{Framework Overview}

The main challenge in the prediction-guided controllers lies in developing an accurate and low-cost model to predict the execution time for each job at runtime. To be accurate enough, this model must capture both input-dependent variations within a program itself and variations from external changes such as interference or platform changes.

In order to capture the input-dependent variations that change program operations, we use off-line profiling to identify control flow features, and program slicing to generate a short code segment that quickly produces those feature values at run-time. More specifically, as the Lasso method was used in the previous work [10], we try to reduce the number of features off-line because the execution time model only requires a subset of all the features. For example, features whose model coefficients are zero can be excluded from the prediction slice. The top part of Figure 4.2 shows these off-line components in my approach. This approach to build a model for input-dependent variations is similar to previous work [10]. Section 3.2 and 4.2 explain these off-line steps in more detail.

However, instead of training this model off-line, I propose to continuously update the model on-line while executing an application as shown in the lower

part of Figure 4.2. For each job, the model is first used to determine the appropriate core and frequency. After the job finishes, the model is updated using the 


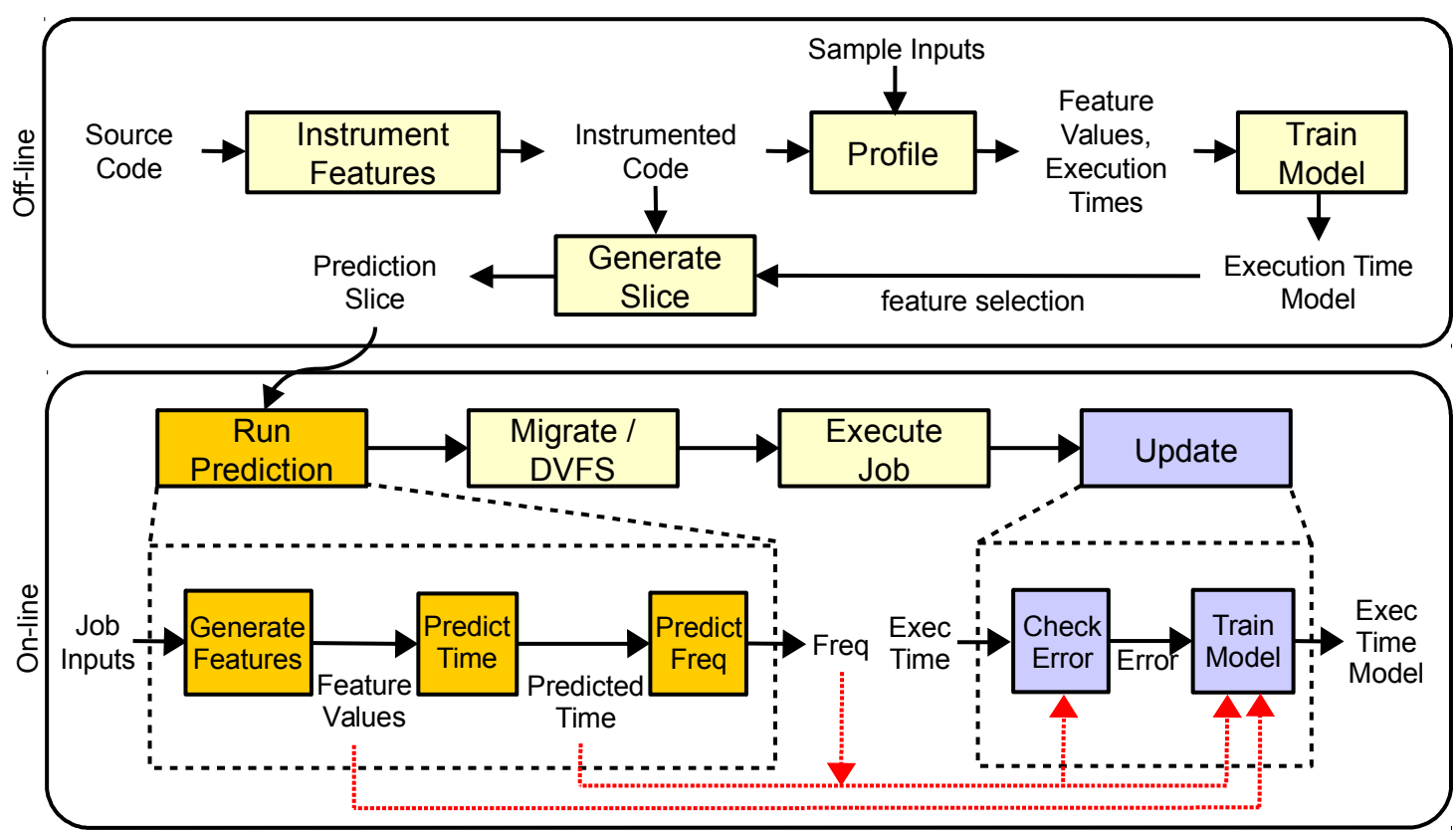

Figure 4.2: Overall flow for the prediction-guided controller with continuous on-line learning.

execution time of that job. This continuous on-line learning represents the main technical innovation in this work, and enables the DVFS controller to adapt to run-time interference and also run effectively on diverse platforms without offline training. In the rest of the chapter, I first describe this on-line training approach focusing only on a DVFS controller. Then, I later extend the approach to migration on heterogeneous cores.

\subsection{Execution Time Prediction Model}

Similar to previous work [10], I generate the feature values using program slicing technique (Section 3.2). With these feature values, we need to predict the execution time. I adopt a simple linear model to map the features to the execution time. In Table 4.1, I summarize the variables and notation used in this 


\begin{tabular}{|c|l|l|}
\hline Variable & Type & Description \\
\hline \hline $\bar{y}$ & Scalar & Predicted execution time \\
\hline$x$ & Vector & Feature values \\
\hline $\boldsymbol{\beta}$ & Vector & Model coefficients \\
\hline \hline$y$ & Vector & A set of actual execution times \\
\hline$X$ & Matrix & A set of generated feature values \\
\hline$X \boldsymbol{\beta}-y$ & Vector & Prediction errors \\
\hline$\|\cdot\|$ & Scalar & L2-norm (sum of squares) \\
\hline \hline$\alpha$ & Scalar & Under-predict penalty weight \\
\hline$W$ & Matrix & A set of prediction penalty weights \\
\hline
\end{tabular}

Table 4.1: Variable and notation descriptions.

chapter. The linear model can be expressed as

$$
\bar{y}=x \boldsymbol{\beta}
$$

where $\bar{y}$ is the predicted execution time, $x$ is a vector of feature values, and $\boldsymbol{\beta}$ are the coefficients that map feature values to execution time. Note that these coefficients $\beta$ consistently need to be updated with newly coming information.

I fit the linear model with a linear least squares approximation. It finds the optimal coefficients, $\boldsymbol{\beta}$, which minimize the mean square error:

$$
\underset{\beta}{\operatorname{argmin}}\|X \beta-y\|^{2}
$$

The $\boldsymbol{\beta}$ that minimizes this can be calculated as:

$$
\beta=\left(X^{T} X\right)^{-1} X^{T} y
$$

Here, each row of $X$ represents feature values for one job with its execution time in $y$. The previous work [10] performed this training off-line. This work shows how the model can be continuously updated at run-time, effectively adding a new row to $X$ after each job, while minimizing overhead and avoiding underpredictions. 


\subsection{On-line Model Training}

The goal in this work is to use on-line learning to continuously update the model presented in the previous section. That is, we want to continuously add control features and execution times from jobs to the $X$ matrix and recalculate the model coefficients $\beta$. In order to build an on-line training model with continuously updated information, there are four major challenges:

1. How to update the model with minimal performance and memory overhead (Subsection 4.3.1).

2. How to avoid under-prediction or deadline misses (Subsection 4.3.2).

3. How to handle the inaccuracy during the initial training phase (Subsection 4.3.3.

4. How to detect and quickly adapt to sudden changes such as unexpected program interference (Subsection 4.3.4.

First, the overhead of updating the model should be as small as possible. If this overhead is too high, then the energy consumed by updating the model could outweigh the energy savings from slowing down the jobs. It could also cause jobs to miss their deadline. Updating the model quickly, however, is not easy due to the fact that as the size of the $X$ matrix grows, calculating the matrix inverse of $X^{T} X$ becomes increasingly expensive. For example, naively running the same training function used for the off-line training on the A7 core took over $80 m s$ even at the highest frequency.

Second, the model should be conservative in order to avoid deadlines misses and achieve good user experiences. Equation (4.1) aims to minimize the sum 
of the absolute errors in the prediction, so it weighs negative and positive errors equally. Positive errors (over-prediction) can result in less energy savings, while negative errors (under-prediction) lead to deadline misses. We, however, are more concerning of under-prediction rather than over-prediction in order to minimize deadline misses. Hence, we need to make the model to be conservative by giving greater penalty to under-prediction.

Third, during the initial training phase, the model will be inaccurate because only a small number of jobs have been seen. If we use this premature model, it is likely to mis-predict the execution time, resulting in high energy usage and/or deadline misses. Instead, we need to check the stability of the model as it is trained and only adopt it when it becomes mature enough.

Finally, as we have discussed in Section 2.2. unexpected events such as program interference can occur which can affect the availability of resources. These effects can cause the trained model to become suddenly inaccurate. Thus, it is necessary for these sudden effects to be detected and for the model to be quickly retrained under the new conditions.

\subsubsection{Fast Updates Using QR Decomposition}

I found that naively porting the off-line training model [10] to on-line does not work due to high overhead. For example, running the off-line training algorithm on the A7 core [21] took over 80ms even at the highest frequency and with a moderate number of features (20) and training jobs (50). Moreover, for online training, the size of the $X$ matrix grows as we add a new row after each

job to include the job in the training set. To limit the increasing computation 
and memory overhead, we may drop old rows from the $X$ matrix and keep its size fixed. However, dropping old samples may negatively impact the model's accuracy.

A better solution is to keep the intermediate result of the least squares approximation (Equation (4.1)). Instead of keeping $X$ and $y$, we can keep $X^{T} X$ and $X^{T} y$ and incrementally updated them:

$$
\begin{aligned}
\tilde{X}^{T} \tilde{X} & =X^{T} X+x_{\text {new }}{ }^{T} x_{\text {new }} \\
\tilde{X}^{T} \tilde{y} & =X^{T} y+x_{\text {new }}{ }^{T} y_{\text {new }}
\end{aligned}
$$

where $x_{\text {new }}$ and $y_{\text {new }}$ represent a new sample, and $\tilde{X}$ and $\tilde{y}$ represent updated $X$ and $y$. This approach enables keeping track of a large number of training samples with a fixed memory footprint. The size of the two matrices $X^{T} X$ and $X^{T} y$ also depends only on the size of $\beta$ and not the size of training set (the number of rows in $X$ ). For example, for $k$ features, the size of $X^{T} X$ and $X^{T} y$ are fixed as $k \times k$ and $k \times 1$. Due to the matrix inversion, however, the computation complexity of this approach is still high $\left(O\left(k^{3}\right)\right)$.

To reduce both memory and computation overhead, I propose to use $\mathrm{QR}$ decomposition [22, 23] to perform fast updates: We apply a QR decomposition to the $X$ matrix as

$$
X=Q R
$$

where $Q$ is an orthogonal matrix $\left(Q^{T}=Q^{-1}\right)$ and $R$ is an upper triangular matrix. By plug this decomposition into Equation (4.1), we can derive:

$$
R \boldsymbol{\beta}=Q^{T} y=d
$$

Then, the coefficients $\beta$ can be easily computed because $R$ is upper-triangular. 
The challenge now becomes how to quickly calculate $R$ and $d$. Note that the matrix $Q$ itself is not necessary to obtain $\beta$ as long as $d$ is available.

For fast training, we keep $R$ and $d$ instead of $X$, and directly update them with a new training sample from the most recent job by adding a row and applying a series of Givens rotations $G=G_{1} G_{2} \ldots G_{k}$. The Givens rotation is a well-known matrix transformation which introduces zeros in matrices. After the Givens rotations, the last row of $R$ always becomes a zero vector and we can remove the row to keep the size of $R$ constant. This method based on the QR decomposition reduces the time complexity to $O(k)$ while keeping the history of all previous jobs with a fixed-size matrix. Appendix $A$ and $B$ further explain the least square approximation problem and QR decomposition with simple examples.

Note that the model is trained on, and thus predicts, execution times assuming the system is running at the maximum frequency. Thus, for jobs that run at a lower frequency, the execution time recorded for training is scaled based on the frequency. The model for scaling execution times based on frequency is discussed in more detail in Section 3.4 and Section 4.4 .

\subsubsection{Conservative Prediction}

Equation (4.1) minimizes the sum of the absolute errors. However, with respect to meeting response-time deadlines, positive and negative errors in predicting the execution time have different effects. Negative errors (under-prediction) may lead to deadline misses, while positive errors (over-prediction) may reduce the energy savings. In order to maintain good user experiences, it is more 
important to minimize deadline misses.

In the previous work [10], we placed a greater penalty on under-prediction by modifying the optimization objective itself (See Section 3.3). It was relatively easy to use the modified objective because we had information for all the samples whether each sample is under-predicted or over-predicted before actually training the model. It is, however, not straightforward to apply the same technique to on-line training model. This is due to the fact that the sample is not given, but the new sample is coming every job, so there is no information of whether the new sample is under-predicted or over-predicted until the sample actually arrives. We, thus, need to determine the case that the new sample is under-predicted at run-time in order to give a higher penalty for such samples.

More specifically, instead of modifying the optimization objective itself, we multiply the under-prediction penalty to such samples at run-time:

$$
\begin{aligned}
& \underset{\boldsymbol{\beta}}{\operatorname{argmin}}\|W(X \boldsymbol{\beta}-y)\|^{2} \\
& W_{i, j}= \begin{cases}\alpha, & i=j \text { and } X_{i} \boldsymbol{\beta}-y_{i}<0 \\
1, & i=j \text { and } X_{i} \boldsymbol{\beta}-y_{i} \geq 0 \\
0, & i \neq j\end{cases}
\end{aligned}
$$

The weight $\left(W_{i, j}\right)$ for a new job is determined based on whether the model had a positive or a negative error when the job is added to the model. The weight does not change afterwards. In this way, the QR decomposition method can be used without any change. $\alpha>1$ is a weighting factor which allows us to place greater penalty on under-predictions. Higher weight can make the model more conservative, but limit energy saving possible. I will discuss the trade-off further in Subsection 5.7.3. 
While the QR decomposition is a known mathematical technique, I believe that it is a new contribution to show how it can be applied to fast on-line training of execution-time models where under-predictions must be avoided even at the cost of over-predictions.

\subsubsection{Initial Training Phase}

Algorithm 1: Algorithm for Checking Stability

1: procedure IS_STABLE (*errors, prev_is_stable)

2: $\quad$ if prev_is_stable $=0$ then

3: $\quad$ is_stable $\leftarrow 1$

4: $\quad$ for $i \leftarrow 1, n_{-}$jobs do

$\triangleright n_{-}$jobs $=10$

5:

if $\operatorname{errors}(i)>$ threshold then

$\triangleright$ threshold $=10 \%$

6: $\quad$ is_stable $\leftarrow 0$

7: $\quad$ break

8: $\quad$ else if prev_is_stable $=1$ then

9: $\quad$ is_stable $\leftarrow 0$

10: $\quad$ for $i \leftarrow 1, n_{-} j o b s$ do

11: $\quad$ if errors $(i)<=$ threshold then

12: $\quad$ is_stable $\leftarrow 1$

13: $\quad$ break

14: $\quad$ return is_stable

The prediction accuracy is likely to be low during the initial training of the model. In particular, while there are fewer sample points than model coefficients, the prediction model is underdetermined and there is not enough in- 
formation to solve the linear system. Thus, we must wait until the model is sufficiently trained before using its predictions for DVFS. For this purpose, we monitor the prediction error by calculating the difference between the measured execution time of jobs and the predicted time. We only consider the prediction model to be stable enough when a number of consecutive jobs all show errors less than a given threshold (see Algorithm 1). From the experiments, I found that checking 10 consecutive jobs and using an error threshold of $10 \%$ work well. Until the model is trained enough, we run jobs at the maximum frequency available in order to avoid deadline misses.

\subsubsection{Fast Adaptation with Explicit Event Detection}

\section{Algorithm 2: Algorithm for Detecting Event}

1: procedure IS_EVENT (is_stable, is_training)

2: $\quad$ is_event $\leftarrow 0$

3: $\quad$ if $i s_{-}$stable $=0 \quad \&$ is_training $=0$ then

4: $\quad$ Remove old data to re-train

5: $\quad$ Use previous model with a higher margin until re-training is done

6: $\quad$ is_event $\leftarrow 1$

7: $\quad$ is_training $\leftarrow 1$

8: $\quad$ return $i$ s_event

* A variable $i s_{-}$training is to distinguish an actual event and unstable model. Initially set as 1 , and become 0 as soon as the model is stable.

* New higher margin depends on error.

In addition to errors during the initial training phase, the prediction model 
can also show large errors when an execution environment changes. For exam-

ple, starting other applications can introduce new interference and terminating applications can remove interference. When such an event happens, we need to be able to quickly retrain the prediction model to reflect the new environmental condition.

For this purpose, my framework monitors accuracy of recent predictions and initiates a retraining event when a certain number (10) of consecutive predictions show large $(>10 \%)$ errors outside the initial training phase. More specifically, we use Algorithm 1 again to detect for instability in the prediction model as the system is running. If instability is detected and we are not in the training phase, then we mark this as an event (see Algorithm 2). When the retraining event is detected, we train a new model from scratch ignoring the existing training data. Alternatively, we could also reduce the impact of old training data over time by giving them lower weights. However, I found that the model is updated too slowly if we keep the old training data.

During the retraining period, we continue to use the old prediction model for running jobs. However, we add a conservative margin by scaling up the predicted execution time by a factor based on the errors observed when the retraining event is detected. More specifically, the average value of the errors observed is added to the previous margin.

\subsection{DVFS Control}

After predicting the execution time, in order to set the appropriate DVFS level, we need to build a model of how the execution time varies as the frequency 


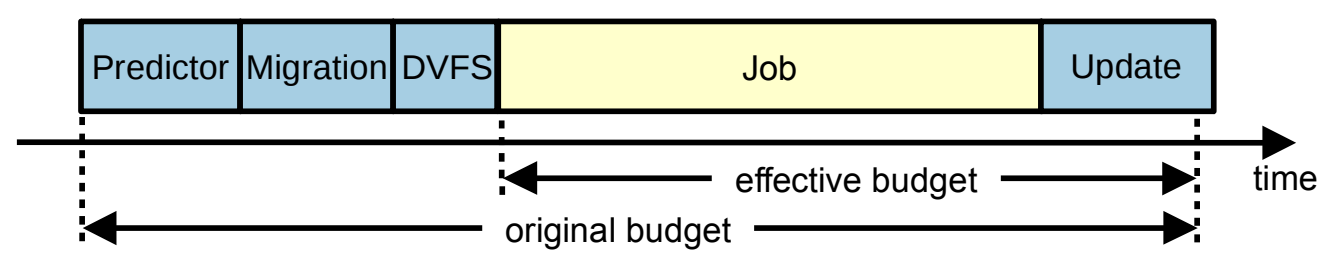

Figure 4.3: The effective budget decreases due to the overhead of updating the model, the predictor, and DVFS switching.

changes. I use the same model described in Section 3.4, but the effective budget is different due to the overhead of performing on-line training and migrating the jobs. Thus, when we consider a time budget, we need to exclude not only the overhead of running the predictor and DVFS switching time, but also the overhead of updating the on-line model and task migration from the original budget (see Figure 4.3):

$$
t_{\text {budget }}=t_{\text {orig_budget }}-\left(t_{\text {unfinished }}+t_{\text {predict }}+t_{\text {migrate }}+t_{D V F S}\right)
$$

where $t_{\text {budget }}$ is the effective time budget, $t_{\text {origbudget }}$ is the original time budget, $t_{\text {unfinished }}$ is the exceeded time from previous job when updating the model exceeds the time budget, $t_{\text {predict }}$ is the overhead of executing the predictor, $t_{\text {migrate }}$ is the task migration overhead, and $t_{D V F S}$ is the DVFS switching overhead.

We can easily get the run-time overhead for executing the predictor, $t_{\text {predict }}$ by simply measuring the actual time. The DVFS switching time, $t_{D V F S}$, and task migration time, $t_{\text {migrate }}$, however, cannot be measured before actually migrating and adjusting to the new frequency. Instead, we use values from microbenchmarking to estimate the DVFS switching time (See Section 3.4). I found that migration overhead is negligible compared to the time scale of millisecond. Detailed analysis for the overhead can be found in Section 5.6 .

We also need to consider the time to update the prediction model. As dis- 
cussed before, we predict the execution time in a conservative manner, so it is likely for most jobs to finish a little bit earlier than deadlines. By using this slack, we can update the model without impacting time budget in most cases. If the slack is not enough, the update reduces the time budget for the next job as in-

dicated by $t_{\text {unfinished }}$ in the equation. As long as the next job finishes before the deadline, there is no impact on user experience. Alternatively, an update may be dropped for cases where there is no slack.

\subsection{Task Migration on Heterogeneous Cores}

Finally, I explore extending the prediction-guided DVFS control methodology to selecting core types in a heterogeneous system. We will use similar terminologies with ARM's big/LITTLE architecture [24]; a little core is a powerefficient core, while a big core is a more powerful but power-hungry core. In essence, I maintain two execution-time prediction models, one for each core type, and choose the more energy-efficient core for a given job. To compare energy-efficiency of cores, we can collect the average power consumption for each core at each frequency running benchmarks. Note that this power model does not need to be specific to applications and can be recorded at run-time with small overhead. For my platform [21], however, I found that simply using the big core only when the little core cannot meet the time budget minimized energy because the little core is almost alway more energy-efficient than the big core.

A naive implementation that trains both prediction models at the beginning of a task can incur unnecessary overhead and is unlikely scale to a larger num- 
ber of core types. Instead, I train the model for a big core on demand only when the big core needs to be used. More specifically, if the model for a little core predicts that the execution time for a job will be longer than the given time budget even at its maximum frequency, I switch to a big core, and train the big-core model. This on-demand training can significantly reduce the training overhead when one core is not necessary. 


\section{CHAPTER 5}

\section{EVALUATION}

This chapter presents the evaluation results for the proposed framework, the prediction-guided controller with continuous on-line learning.

\subsection{Experimental Setup}

I ran most experiments on an ODROID-XU3 [21] development board with Ubuntu 14.04. The ODROID-XU3 includes a Samsung Exynos5422 SoC with 4 ARM Cortex-A15 and 4 Cortex-A7 cores (Table 5.1).

\begin{tabular}{|c|l|l|}
\hline & Cortex-A15 & Cortex-A7 \\
\hline \hline Instruction set & ARMv7-A & ARMv7-A \\
\hline Cores & 4 & 4 \\
\hline L1 cache & $64 \mathrm{~KB}(32 \mathrm{~KB}$ I\$, 32KB D\$) & 64KB (32KB I\$, 32KB D\$) \\
\hline L2 cache & 2MB & 512KB \\
\hline Feature & OOO, 15-stage pipleline & In-order, 8-stage pipleline \\
\hline Frequency Level & 200Mhz-2.0Ghz (100Mhz step) & 200Mhz-1.4Ghz (100Mhz Step) \\
\hline
\end{tabular}

Table 5.1: Microarchitecture of Cortex-A15 and Cortex-A7 clusters.

I show results of energy and deadline misses on the more power-efficient A7 core, but I saw similar trends on the A15 core. I measured power using built-in sensors with a sampling rate of 213 samples per second and integrated over time to calculate energy consumption. I use both A15 and A7 cores for heterogeneous system experiments. To compare the accuracy of my on-line training scheme on different platforms, I use both A7 and A15 core, as well as two different Intel cores.

I use a set of nine benchmark applications, including three games, a web browser, speech recognition, a video decoder and three applications from the 
MiBench suite [25]. Table 5.2 provides details of the benchmarks, including the number of program features used.

\begin{tabular}{|c|c|c|}
\hline Benchmark & Description & \# of Features \\
\hline 2048 [1] & $\begin{array}{l}\text { Puzzle game, } \\
\text { Update and render one turn }\end{array}$ & 27 \\
\hline curseofwar [26] & $\begin{array}{l}\text { Real-time strategy game, } \\
\text { Update and render one game loop iteration }\end{array}$ & 2 \\
\hline ldecode [27] & $\begin{array}{l}\text { H.264 decoder, } \\
\text { Decode one frame }\end{array}$ & 9 \\
\hline pocketsphinx [28] & $\begin{array}{l}\text { Speech recognition, } \\
\text { Process one speech sample }\end{array}$ & 1 \\
\hline rijndael [25] & $\begin{array}{l}\text { Advanced Encryption Standard (AES), } \\
\text { Encrypt one piece of data }\end{array}$ & 18 \\
\hline sha [25] & $\begin{array}{l}\text { Secure Hash Algorithm (SHA), } \\
\text { Hash one piece of data }\end{array}$ & 22 \\
\hline stringsearch [25] & $\begin{array}{l}\text { Search for words in phrases, } \\
\text { Perform a set of searches }\end{array}$ & 4 \\
\hline uzbl [29] & $\begin{array}{l}\text { Web browser, } \\
\text { Execute one command (e.g., refresh page) }\end{array}$ & 13 \\
\hline xpilot [30] & $\begin{array}{l}\text { 2D space game, } \\
\text { Update and render one game loop iteration }\end{array}$ & 78 \\
\hline
\end{tabular}

Table 5.2: Benchmark descriptions and the number of features used.

\subsection{DVFS Controllers}

I compare the proposed prediction-based DVFS controller and core scheduler with existing DVFS controllers and a recently proposed PID control scheme. Specifically, I measure results for the following schemes:

1. performance: The Linux performance governor [11] sets the CPU speed to the maximum frequency. I normalize the energy results to this scheme. 
2. interactive: The Linux interactive governor [11] was designed for latencysensitive, interactive applications. It sets the frequency based on CPU utilization every 80 milliseconds by default and changes to highest frequency if the CPU load exceeds $85 \%$.

3. pid: The PID-based controller calculates the error with a PID feedback mechanism to predict the execution time of the next job [12]. I set PID parameters for each benchmark by training them separately offline, thus optimized to reduce deadline misses.

4. offline: This is the prediction-based DVFS controller that trains the prediction model off-line [10].

5. online: This is the prediction-based controller with on-line training as described in this thesis.

\subsection{Energy Savings and Deadline Misses}

Here, we study the impact of the proposed on-line training on the energy savings and deadline misses when used for the DVFS control on one core without migration. Jobs are run with a time budget of 50 milliseconds as running faster than this is not noticeable to the user [5,6]. pocketsphinx takes at least 100s of milliseconds to run (see Table 5.2) so I use a 4 second deadline. This corresponds to the time limit that a user is willing to wait for a response [4]. Energy numbers are normalized to the energy usage of the performance governor. Deadline misses are reported as the percentage of jobs that miss their deadline. 

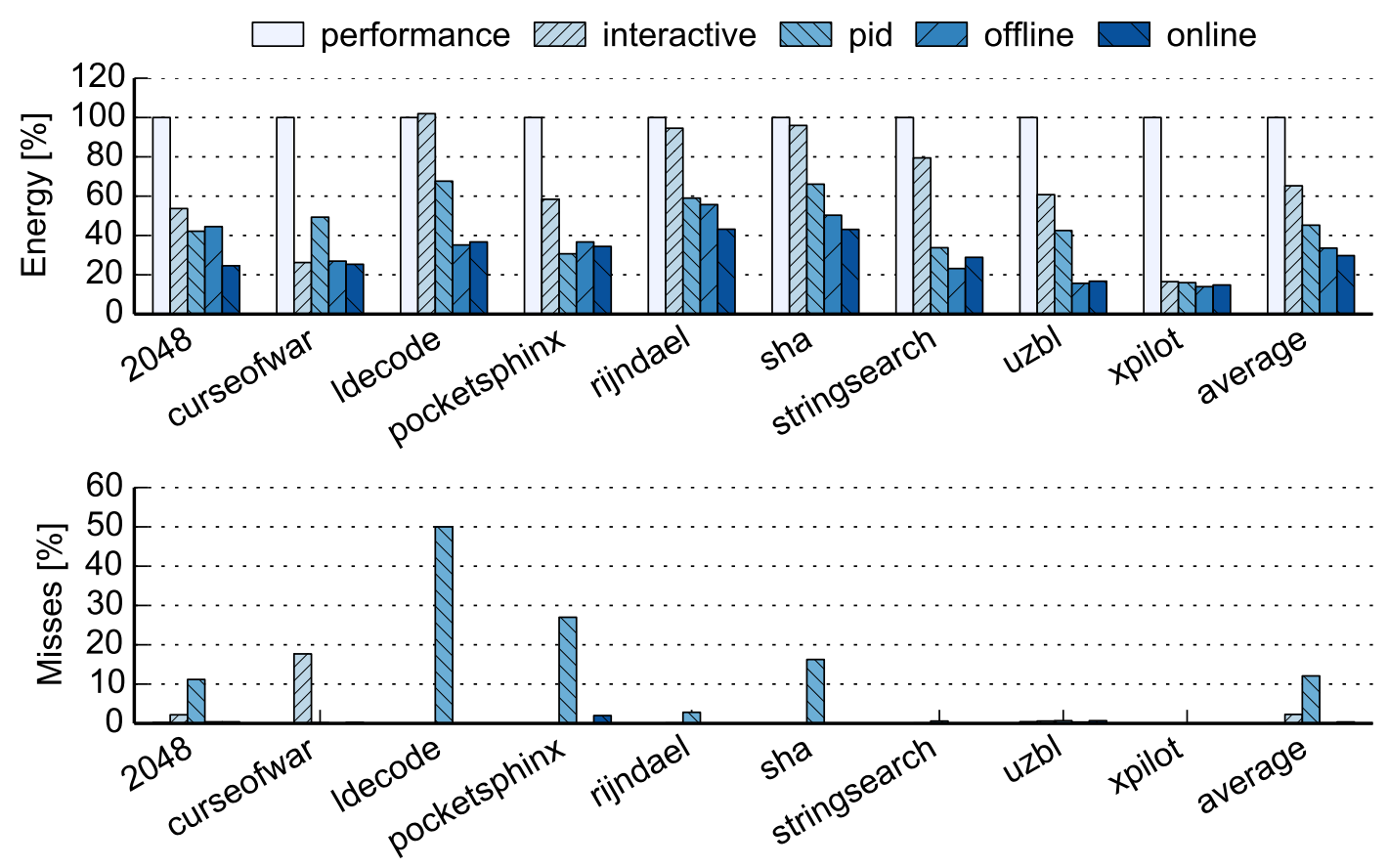

Figure 5.1: Normalized energy usage and deadline misses without interference.

\subsubsection{Without Interference}

Figure 5.1 shows the results when each benchmark runs without interference. Here, I pinned a benchmark to the A7 core while using A15 cores to run OS and background jobs. No other application was running. For on-line training, the results include the initial training overhead before the model becomes stable.

On average, the on-line training scheme achieves significant energy savings compared to traditional DVFS controllers: $70.3 \%$ savings over the performance governor, $36.6 \%$ savings over the interactive governor, and $15.5 \%$ savings over the PID-based controller. The energy consumption is also slightly lower (3.9\%) than that of the off-line scheme. This is because the prediction model is more accurate with on-line training, which continuously trains the model with runtime samples. Both off-line and on-line training schemes show less than $0.5 \%$ 
deadline misses on average across all benchmarks, indicating their prediction is quite accurate without interference. On the other hand, the interactive governor and the PID-based controller both show a large number of deadline misses because their prediction is not accurate.

\subsubsection{With Interference}

Figure 5.2 shows the results when interference is introduced in the middle (after 3 seconds). Here, to introduce interference, I ran a set of dummy applications that run compilations. More specifically, I ran two cpu-intensive compilations using a gcc compiler on the same core where a benchmark is running. The dummy applications run until the end of a benchmark, which accounts for $60 \%$ to $80 \%$ of each benchmark run on average. To be fair, the same dummy applications are inserted to all workload at the same point with an automated script.

The experimental results with interference clearly show the limitation of the off-line training scheme. Because the model with off-line training cannot adapt to run-time interference, it often under-predicts the execution time and misses the deadlines. On the other hand, the on-line training scheme maintains low deadline misses (about 3\%) even with unexpected interference. At the same time, the on-line training scheme still provides significant energy savings over other DVFS controllers: $57.7 \%$ over the performance governor, $23.8 \%$ over the interactive governor, and $4.7 \%$ over the PID-based controller.

Figure 5.3 compares the predicted and actual execution times when running sha for off-line and on-line training schemes. As shown, the off-line training 

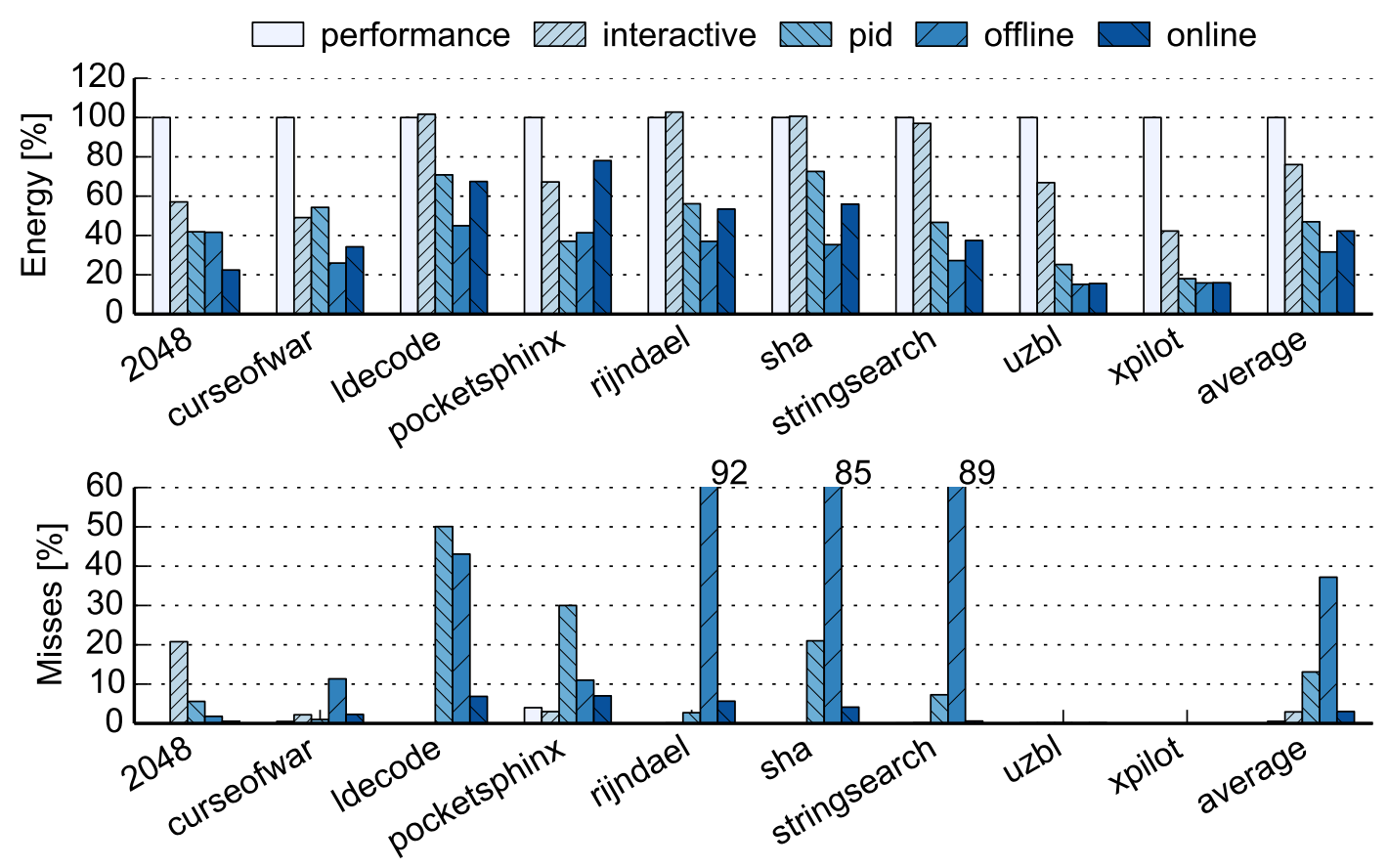

Figure 5.2: Normalized energy usage and deadline misses with interference.

scheme cannot adjust to the increased execution time once interference begins. On the other hand, the on-line training scheme can adapt after seeing several jobs. Note that the on-line training scheme can also experience some deadline misses for a short period right after the interference is introduced because my scheme only initiates the fast re-training after experiencing significant errors for 10 consecutive jobs. In the figure, the model detects the interference event at Job 30.

In some cases, deadline misses are unavoidable with heavy interference. For example, the results for pocketsphinx show deadline misses even for the performance governor, which always runs at the highest frequency. In these cases, we can leverage heterogeneous cores and run a job at a high-performance core to avoid deadline misses. 

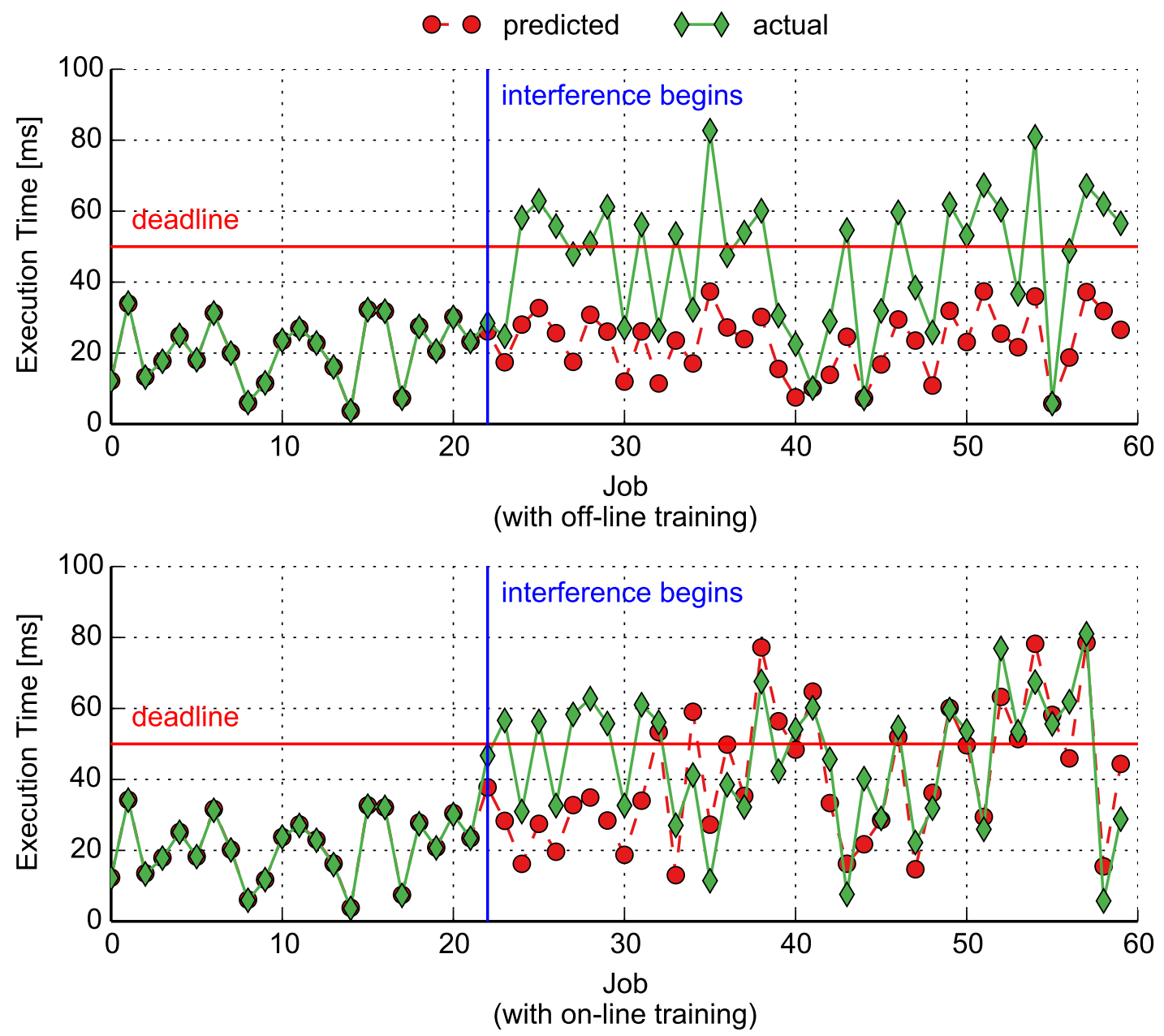

Figure 5.3: Predicted and actual execution times for off-line (top) and online (bottom) training schemes with interference (sha).

\subsection{Heterogeneous Cores}

Figure 5.4 shows the experimental results when both A7 and A15 cores are used. In this case, the prediction-guided controller can migrate a job between the two types of cores. In order to migrate the job to specific core, I used the Linux CPU affinity system call (sched_setaffinity). This function takes pid (process ID) and mask (CPU affinity mask). And, the process specified by pid is migrated to the CPU specified in mask. For these experiments, in order to study cases where 

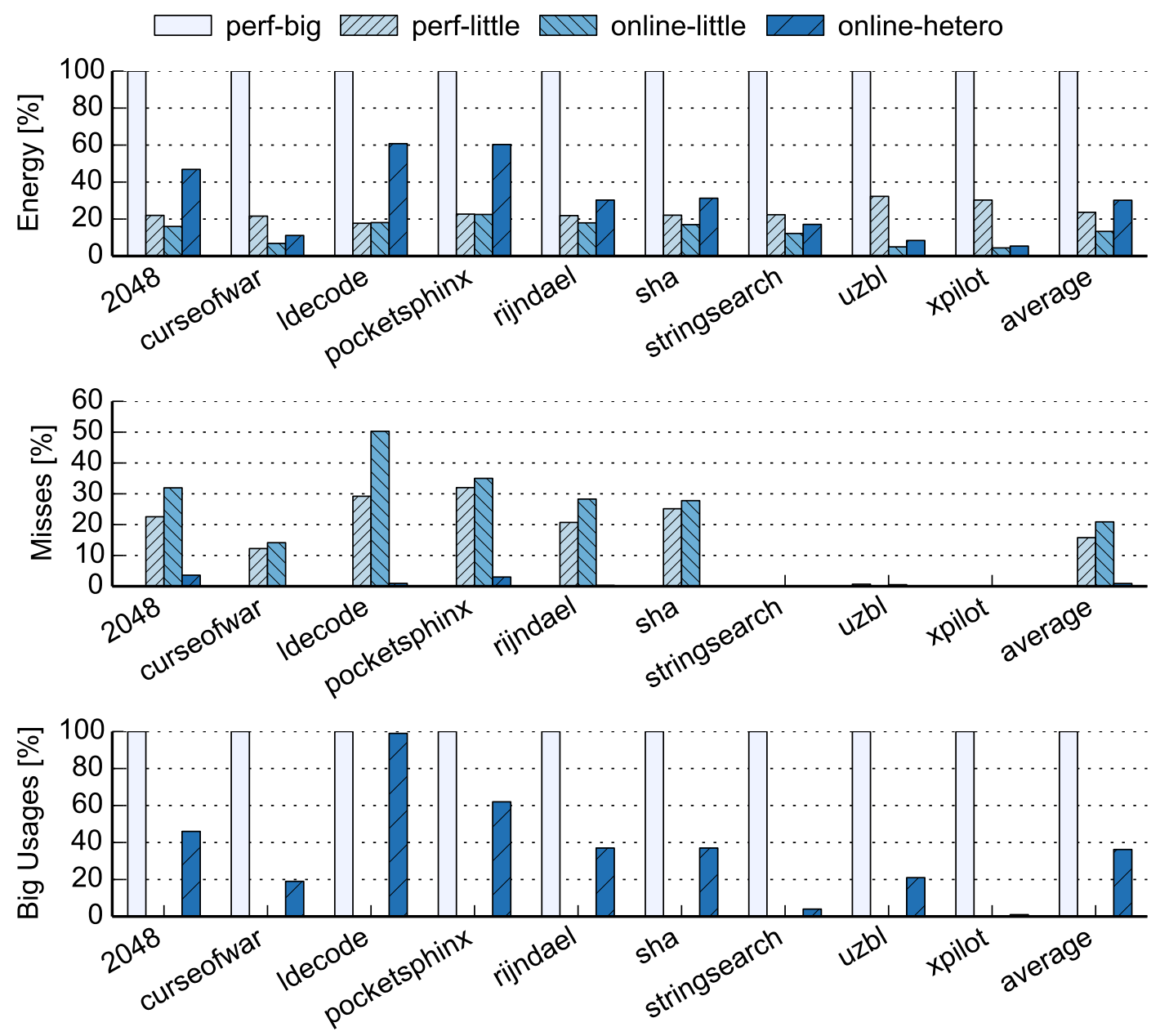

Figure 5.4: Normalized energy usage, deadline misses, and big core usages with heterogeneous cores.

the A7 core is not enough to meet deadlines, I reduced the time budget by $40 \%$ so that the A7 can only meet the deadline for a subset of jobs. As a result, even the performance governor running at the highest frequency experiences many deadline misses when a benchmark only runs on the little core (perf-little). Similarly, the on-line training scheme on the little core (online-little) results in many deadline misses. On the other hand, the on-line training scheme using both cores can effectively remove almost all deadline misses by selectively using the big core when necessary. Because the power consumption of the big core is significantly higher than that of the little core, the heterogeneous core 
configuration consumes $6.5 \%$ and $16.9 \%$ more energy compared to the performance governor and the on-line training on a little core. However, because the prediction-guided controller still uses the little core when it is fast enough to meet deadlines, the on-line training scheme on heterogeneous cores still consumes significantly $(69.8 \%)$ less energy compared to the performance governor on the big core.

\subsection{Prediction Accuracy}

Here, I provide more detailed discussion on the prediction accuracy of both offline and on-line training schemes.

\subsubsection{Without Interference}

Figure 5.5 shows the prediction error when there is no interference. In box-andwhisker plots, the box represents the first and third quartiles and the line in the box marks the median value. Outliers (values over 1.5 times the inner quartile range past the closest box end) are marked with an " $x$ " and the non-outlier range is marked by the whiskers. Due to heavier under-prediction penalty, the prediction skews toward over-prediction with average errors higher than 0 . For both off-line and on-line training, we can see prediction errors of less than $5 \mathrm{~ms}$, which is only $10 \%$ of a $50 \mathrm{~ms}$ time budget. ldecode shows higher prediction errors, which limits energy savings. pocketsphinx (not shown) has errors ranging from $40 \mathrm{~ms}$ to $1500 \mathrm{~ms}$ of over-prediction with an average of $960 \mathrm{~ms}$ over-prediction. Although these errors are larger in absolute terms than the other benchmarks, 


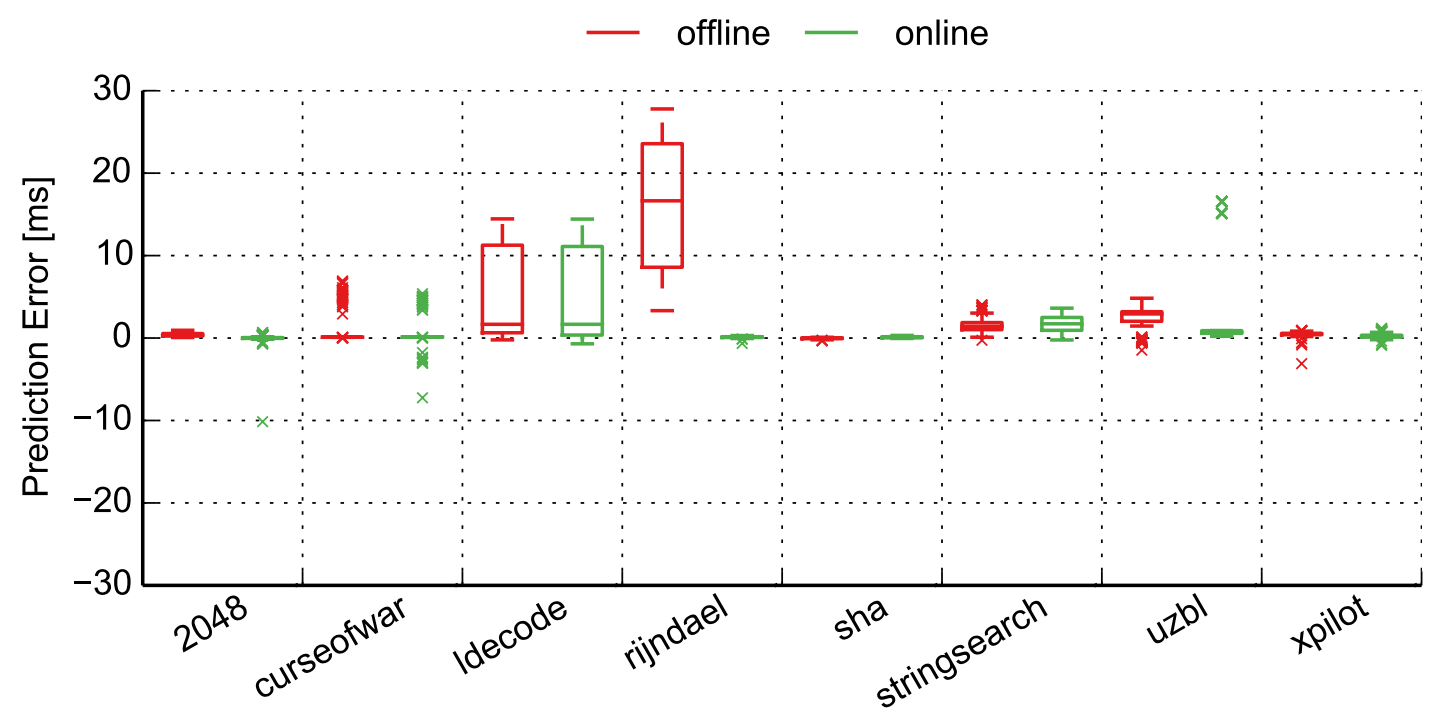

Figure 5.5: Prediction errors for off-line and on-line training without interference.

they are on the same order of magnitude when compared to the execution time of pocketsphinx jobs $(4 s)$. Overall, the results suggest that the on-line training scheme has a comparable or lower error rate compared to the off-line scheme.

\subsubsection{With Interference}

Figure 5.6 shows the prediction errors when there is interference. Although the error range of some benchmarks increases due to the interference, the on-line training scheme can still provide mostly conservative execution time prediction. On the other hand, the off-line training scheme significantly under-predicts the execution time for most benchmarks, leading to deadline misses. 


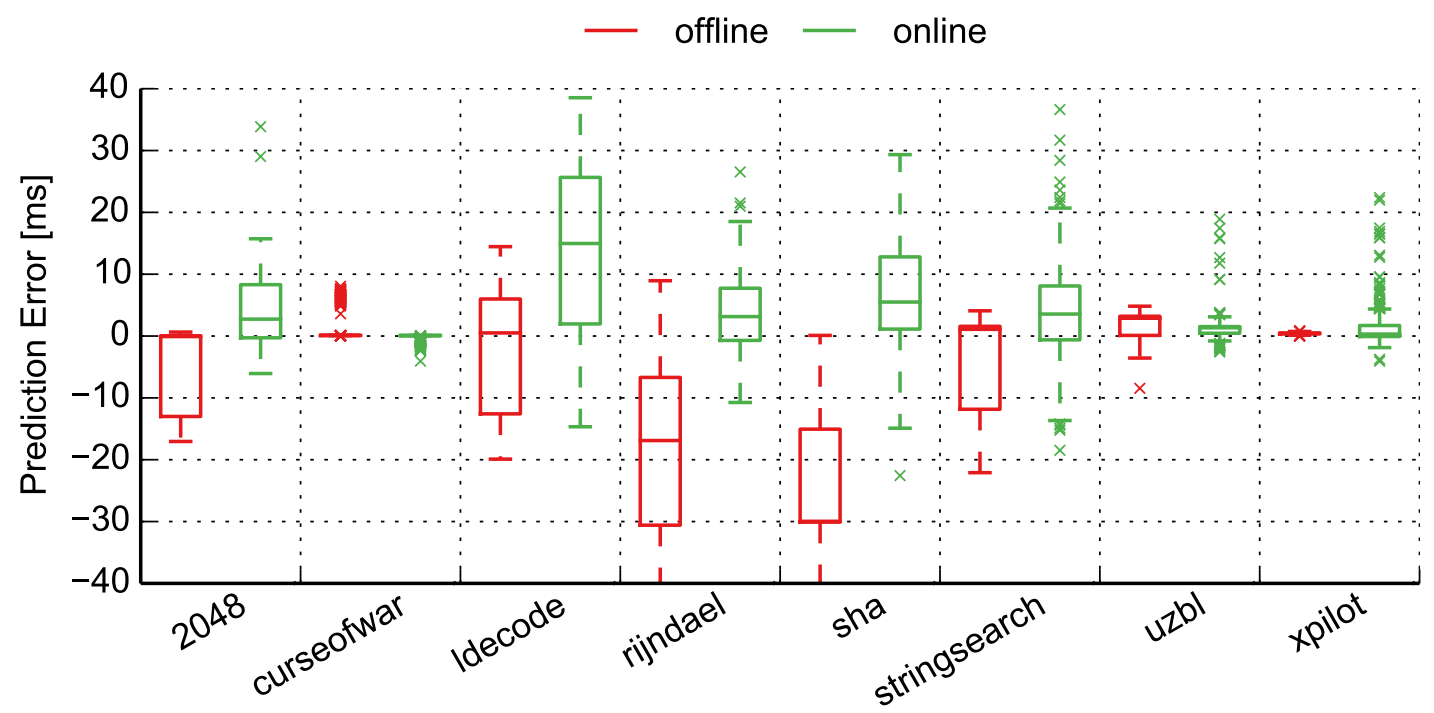

Figure 5.6: Prediction errors for off-line and on-line training during interference.

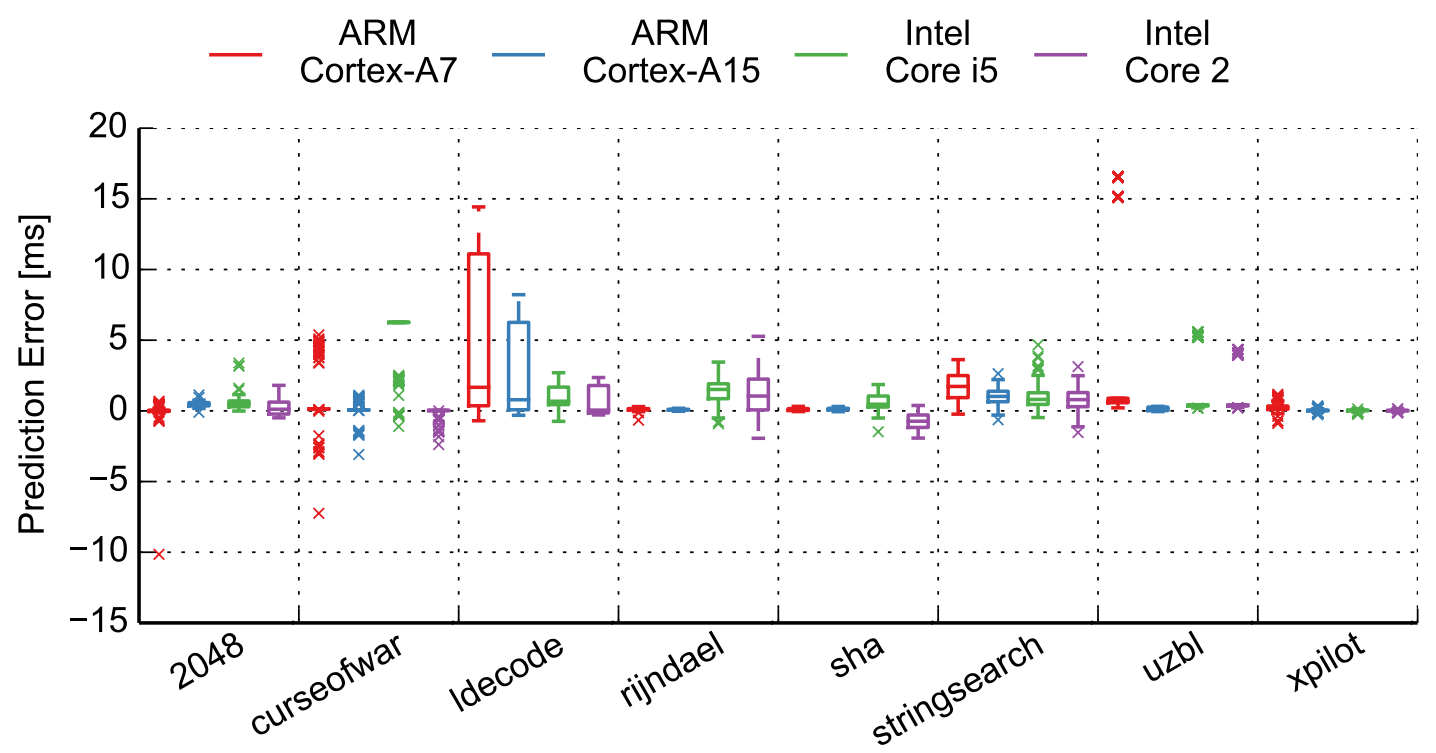

Figure 5.7: Prediction errors for on-line training on different processors.

\subsubsection{Multiple Platforms}

Figure 5.7 shows the prediction errors for the on-line training scheme on multiple processors. Here, the same program features are used for all cases, while 
the model itself is trained on each core at run-time. The results show that my execution time prediction model with on-line training can provide fairly accurate estimates across a range of different hardware platforms. In most cases, the prediction error is less than $5 \mathrm{~ms}$, which is less than $10 \%$ of a typical time budget. Moreover, most errors come from over-predictions, which do not lead to deadline misses. The results suggest that my prediction model can be easily ported to many platforms without explicit off-line training.

\subsection{Analysis of Run-time Overheads}

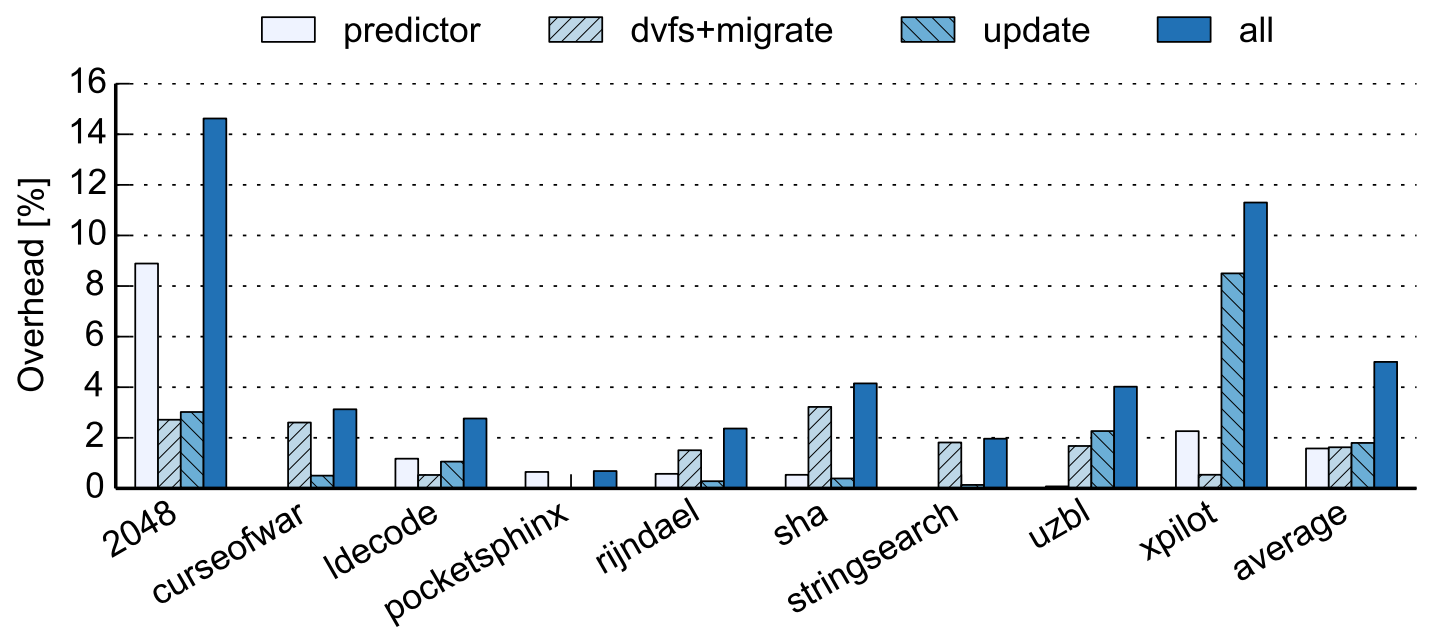

Figure 5.8: Average overhead of the predictor, migration, DVFS switching, and updating the model.

Figure 5.8 shows the average overhead for executing the predictor, switching core/DVFS, and updating the model. Overall, the results show that the update overhead, which represents the overhead of the on-line training over the offline training, is quite low; less than $2 \%$ of the time budget on average. The overhead of updating the model is higher for xpilot compared to others due to a large number of features. The overhead can potentially be lowered by only 
performing the update less frequently rather than after every job.

For 2048, the overhead of predictor is higher than other benchmarks, because the core often runs at a low frequency (jobs are short). I can lower this overhead by running the predictor at a higher frequency if necessary.

Note that I measured the migration overhead combined with the DVFS switching overhead because it is very small. ARM's technical report [31] indicates that the migration overhead as low as 20,000 cycles, which corresponds to $20 \mu \mathrm{s}$ at $1 \mathrm{GHz}$. In the experiments, I found the average overhead to be around $60 \mu s$, which is still negligible.

\subsection{On-line Training Sensitivity Studies}

\subsubsection{Accuracy vs. Number of Samples}

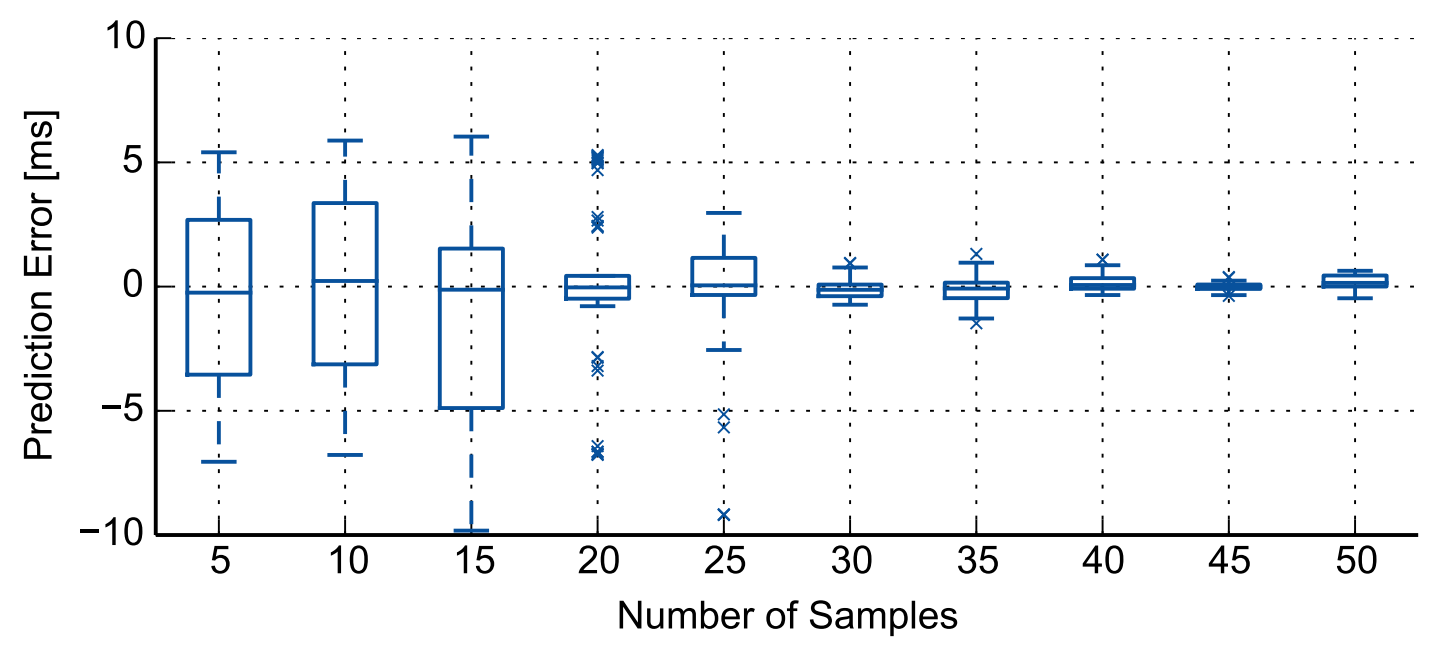

Figure 5.9: Prediction errors as the number of samples for training is varied (2048). 
Figure 5.9 shows how the accuracy of the model changes as the number of samples to train the model is varied for the 2048 game. The results show that the model becomes more accurate with the increasing number of samples, but the benefit is minimal after 30 to 35 samples. The trend is the same of other benchmarks, and a few tens of samples are sufficient for most benchmarks.

\subsubsection{Impact of Interference Intensity}

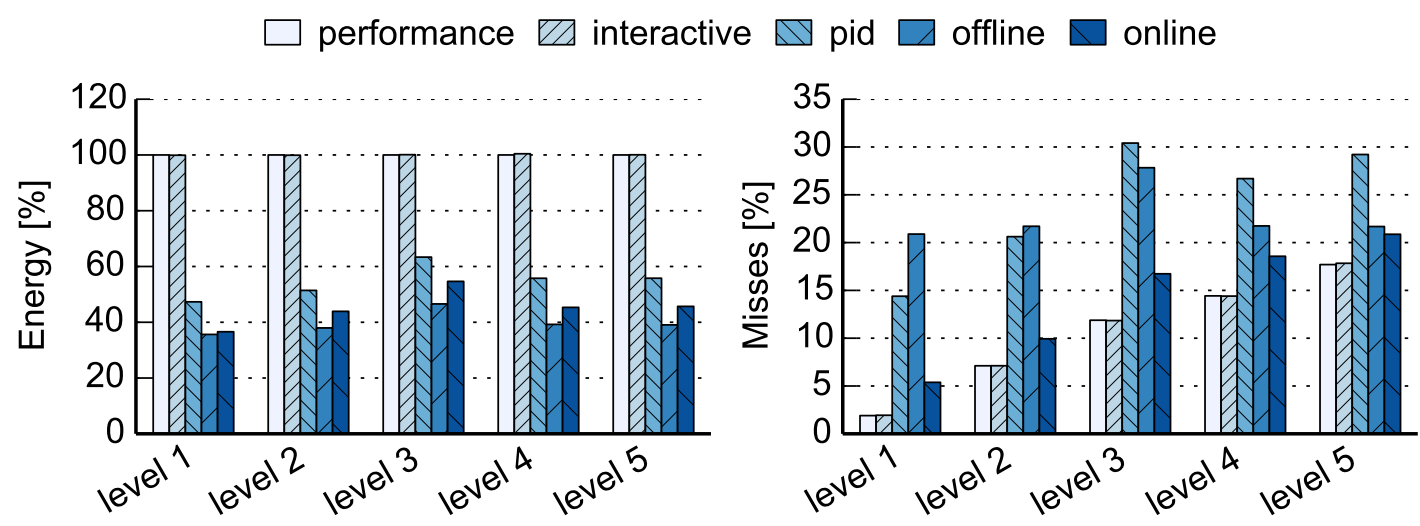

Figure 5.10: Normalized energy usage and deadline misses as the intensity level of interference is varied.

Figure 5.10 shows how the energy savings and deadline misses vary as the intensity level of interference increases. The result shows the average across all the benchmarks. Here, the intensity is increased by adding more dummy applications. For example, I ran one dummy application for level 1 and two dummy applications for level 2, and so on. As expected, we can see that the number of deadline misses for all the controllers increases as the intensity level gets higher, but the on-line training scheme can still save energy with a small increase in deadline misses compared to the performance governor. 


\subsubsection{Under-prediction Trade-off}

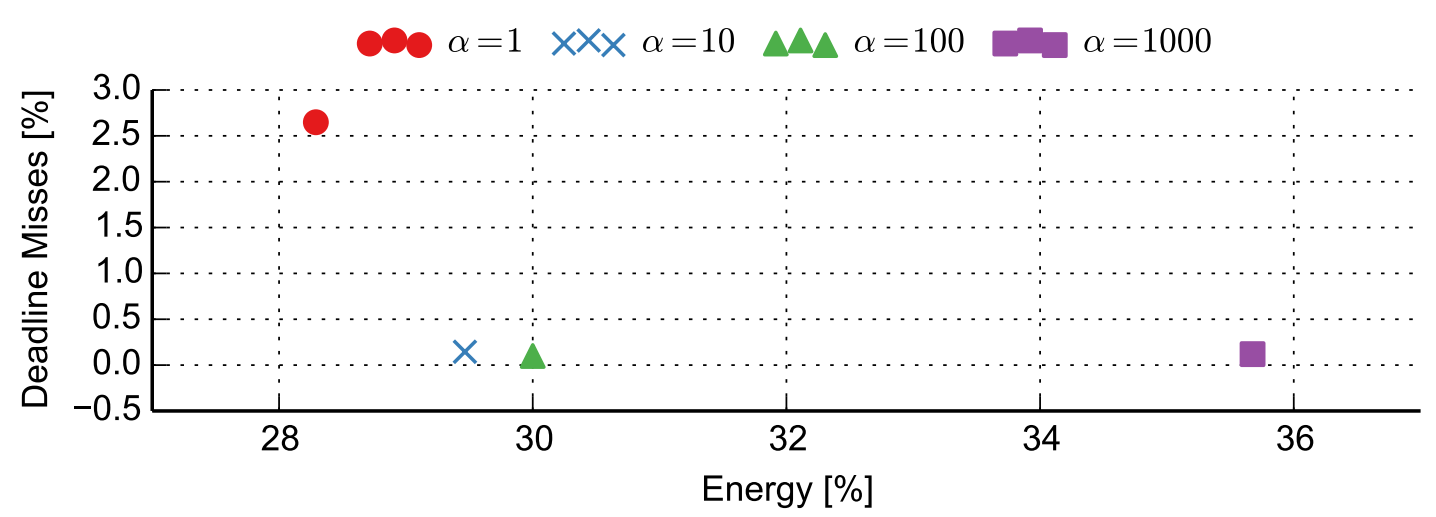

Figure 5.11: Normalized energy usage and deadline misses as the underprediction penalty weight $(\alpha)$ varies.

Subsection 4.3.2 discusses how my scheme makes predictions more conservative by placing a higher weight $(\alpha>1)$ on under-prediction. Figure 5.11 shows how the energy savings and deadline misses change as we vary the under-prediction penalty from 1 to 1000 . The result indicates the average value across all the benchmarks. The results show that the under-prediction penalty provides a different trade-off point between energy and deadline misses. As the weight increases, the energy consumption increases while deadline misses decrease. I found that $\alpha=100$ provides a good trade-off point, and use this value for other experiments.

\subsection{Idle Power}

In Section 5.3, we showed the result of energy on A7 cores. This energy, however, includes all four A7 cores within the A7 cluster because the built-in sensors cannot measure the power for each core separately. 


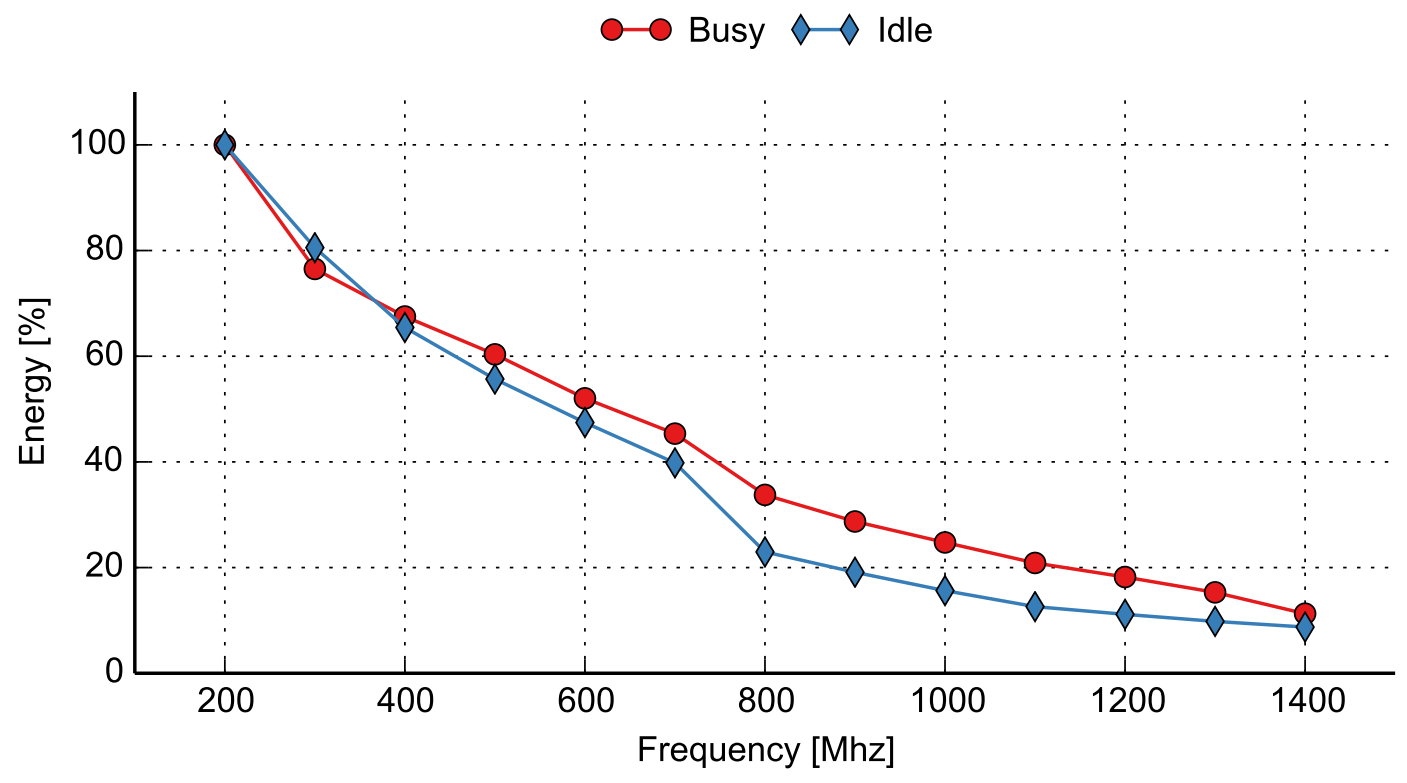

Figure 5.12: Normalized energy usage for busy and idle cores (Normalized to maximum frequency of each case separately).

Since I ran the benchmarks on single core, only this core is busy and the remaining three cores are actually in idle state. Thus, if the power of idle cores shows a different behavior from the power of busy cores when we set the different DVFS levels, the result we showed can be inaccurate.

Figure 5.12 shows the normalized energy usage for both busy and idle cores. For busy, I measure the average power of running our benchmarks while sweeping the frequency level. I ran the benchmarks on all four cores at the same time. For idle, I measure the power of four idle cores. I normalized the energy usage of busy cores to the maximum frequency on busy. Similarly, I normalized the energy usage of idle cores to the maximum frequency on idle. As shown in the figure, the relative ratio of energy consumption between two DVFS levels is similar for both busy and idle cases. The result in Section 5.3, therefore, is reasonable.

For more information, I normalized all the energy usage to the maximum 


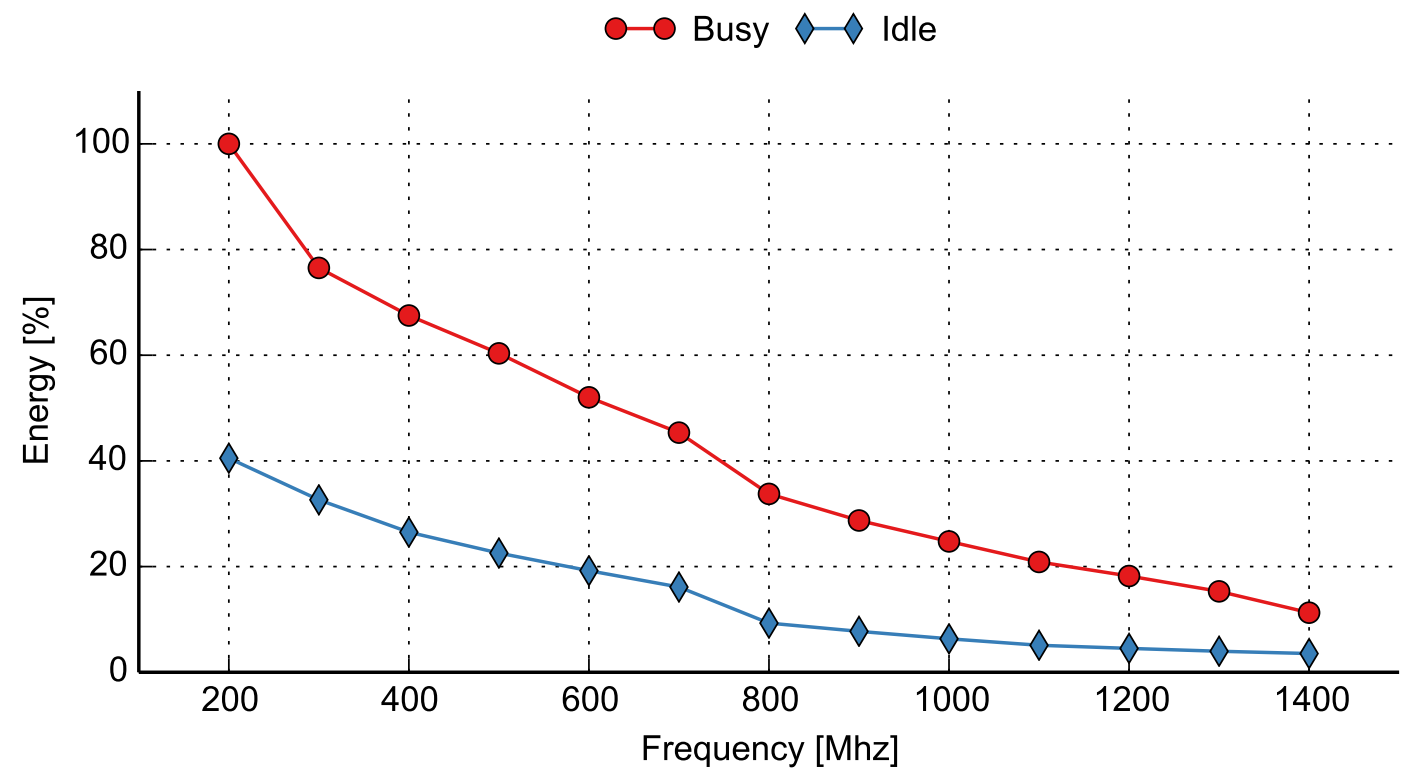

Figure 5.13: Normalized energy usage for busy and idle cores (Normalized to maximum frequency of busy).

frequency on busy (See Figure 5.13). From this graph, we can compare the energy consumption between both cases. 


\section{CHAPTER 6}

\section{RELATED WORK}

\subsection{Dynamic Voltage and Frequency Scaling}

There exist many DVFS controllers. Most controllers look to decrease voltage and frequency when the performance impact is minimal. For example, the builtin Linux governors [11] adjust DVFS based on CPU utilization. However, these controllers do not take into account performance requirements or deadlines.

A number of reactive DVFS controllers have been proposed that use the past history of job execution times to predict the execution time of future jobs. Choi et al. [13] used moving averages of job execution time history to predict execution times for an MPEG decoder. Similarly, Pegasus [14] used instantaneous and average job execution times to make DVFS decisions. Nachiappan et al. [15] used a moving average to set DVFS for multiple IP cores. Gu and Chakraborty [12] used a PID-based controller to predict execution times of frames in a game. These history-based, reactive controllers are not able to adapt fast enough to job-to-job variations in execution time, resulting in either high energy usage or deadline misses.

Recent work has shown that prediction-based controllers can outperform these reactive controllers. For example, a number of prediction-based controllers have been designed for specific applications. Gu and Chakraborty [7] predicted the rendering time for a game frame based on the number of objects in the scene. Zhu et al. used prediction-based control to select core and DVFS levels for a web browser based on HTML and CSS features [8] and event types 
[6]. Adrenaline [9] looked to reduce the tail latency of datacenter applications including web search and Memcached by classifying jobs by query type. Lo et al. [10] showed how prediction-based DVFS controllers could be generated generally for applications by using program slicing. However, their approach cannot adapt to run-time events and requires an off-line training phase which makes it expensive to port to new platforms. Our work extends their predictionbased DVFS controller with on-line learning capabilities to automatically adapt to run-time changes.

\subsection{Execution Time Prediction}

Worst-case execution time analysis is a well-studied problem in hard real-time systems [32]. This analysis looks at the problem of estimating execution time of a program in the worst-case. This can be used as a conservative bound for setting DVFS in order to meet deadlines, but it does not predict run-time changes in job execution time. In addition, it requires the analysis to be redone for each target platform.

Mantis [18] and Lo et al. [10] have shown how program slicing can be used to predict input-dependent variations in execution time. Their work requires expensive off-line training in order to train the prediction model. In this work, we have shown how to account for platform changes and run-time interference by using continuous on-line learning.

ATLAS [33] looked at predicting execution time in the context of soft realtime scheduling. Similar to our approach, their prediction model is also contin-

uously updated online. However, ATLAS requires programmer instrumenta- 
tion in order to predict input-dependent variations while our approach is able to automatically generate these features from the program source. In addition, we show how this prediction can be used for DVFS control and task migration to save energy.

\subsection{Heterogeneous Core Management}

Similar to the work on DVFS, most work on scheduling for heterogeneous systems seeks to use a more power-efficient core when the performance impact is low [34, 35, 36]. PIE [37] proposed the mechanism that collects profiling data while executing the applications on one core type to estimate the performance and power consumption on the other core type. These schemes do not take into account the response-time requirements of the application. Similarly, there have also been reactive core selection algorithms [38].

Zhu et al. proposed off-line [8] and on-line [6] schemes for selecting DVFS and core type for a web browser. Their prediction is based on features that are specific to web browsers, making it difficult to generalize to other applications. Our work can be applied across a range of applications. 
CHAPTER 7

POSSIBLE EXTENSIONS

This chapter discusses possible extensions and future works in the context of predictive DVFS domain.

\subsection{Parallel Job Execution on Multi-core Systems}

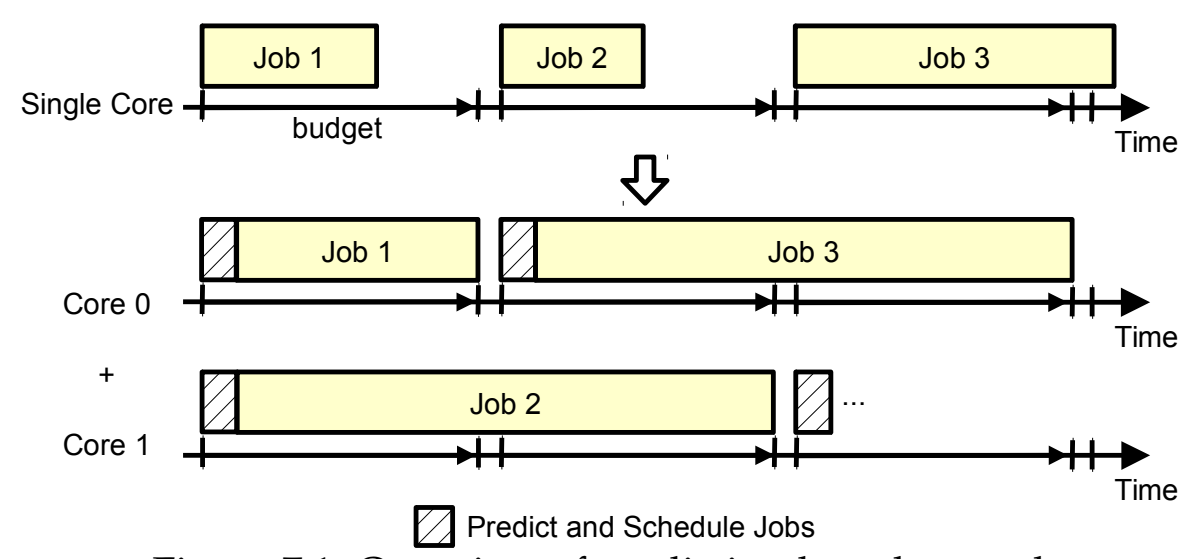

Figure 7.1: Overview of prediction-based control.

In this section, we will discuss about potential benefits by running jobs in a parallel manner on multi-core systems to decrease the DVFS level even more compared to single-core systems. We will study different scheduling algorithms and how we are able to leverage a multi-core system to schedule jobs between cores to further decrease energy usage. Figure 7.1 shows an overview of the proposed prediction-based controller's operation on multi-core systems. I tested the prototype using one benchmark from the MiBench suite. Compared to running on single-core, I obtained energy savings of around 15\% with no deadline misses. More importantly, running on multi-core systems can effectively remove deadline misses when the time budget is shorter. 


\subsubsection{Motivational Example}

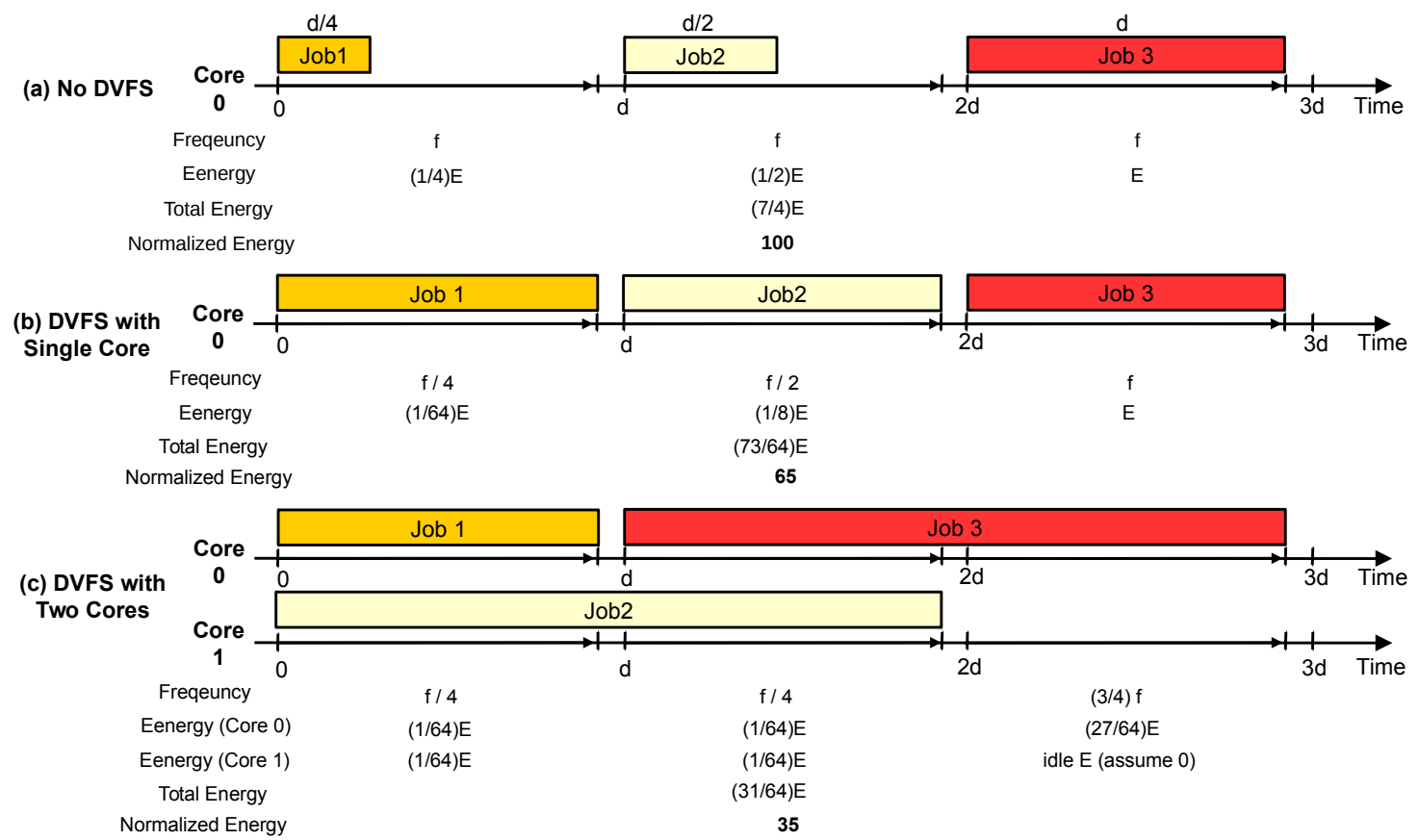

Figure 7.2: Motivational example ((a) no DVFS : This is run at maximum frequency $f,(b)$ DVFS with single core : This scales the frequency to just meet the deadline, and (c) DVFS with two cores : This not only scales the frequency, but also schedules the jobs to multiple cores).

I introduce a motivational example to show how this extension can save more energy compared to previous work on single core case [10] in Figure 7.2 In this example, we refer $f$ as the maximum frequency, $d$ as a time budget (i.e. deadline time), and $E$ as a normalized energy for the job which takes $d$ time to finish at frequency $f$. We also use the following equations to calculate approximate energy for each job:

$$
\begin{gathered}
E n=P t \\
P \propto V^{2} F, \quad V \propto F, \quad P \propto F^{3}
\end{gathered}
$$

where $E n$ is an energy, $P$ is a power, $V$ is a voltage, and $F$ is frequency. 
First, case (a) shows original job sequences with no DVFS. Essentially, this represents the Linux Performance governor running at maximum frequency all the time. Let us assume that Job 1,2 and 3 takes $d / 4, d / 2$, and $d$ time, respectively. As all the jobs run at the highest frequency $f$, total energy is $(7 / 4) E$. Second, case (b) shows when setting the frequency to just meet the deadline. We assume that the execution time is linearly proportional to the frequency. For example, Job 2 runs $2 x$ longer when the frequency $f$ becomes in half. As we can decrease the frequency, the energy consumption decrease to (73/64)E. Finally, case (c) shows when we extend case (b) to two cores. As we can schedule Job 2 earlier, the frequency can decrease even further, which results in more energy savings $((31 / 64) E)$. In brief, if we normalize the total energy to case (a), multi-job systems (case (c)) can save 65\% energy more than the case (a), and this is $46 \%$ energy savings compared to the case (b). Although, there can be idle energy and some overhead such as setting new DVFS level, we argue that the energy savings by decreasing the frequency can outweigh such overheads. This further energy savings mainly motivate this extension.

\subsubsection{Assumption}

After predicting the execution time and the frequency with a similar way [10], we need to schedule the job to multiple cores accordingly. To run the job in parallel on multiple cores, we set two main assumptions:

1. The inputs for future jobs can be predicted beforehand.

2. There is no dependency between each job. 
First, we assume that we can predict the input for future jobs to predict the execution time for multiple jobs beforehand. For the steaming applications like decoders, this can be possible. Since most streaming applications queue the job before actually running the job. This can be challenging, however, for general interactive applications such as games or web browsers, as we cannot predict the future input from actual users.

Seconds, we assume that there is no dependency between every job. Since we need to execute the multiple jobs as the same time, we need to carefully consider whether current job is dependent on any other jobs. For a simple design, we assume that there is no such cases, but in more complicated benchmarks, this is likely to happen. For example, in Figure 7.1. Job 1 and Job 2 should be independent. If any variables in Job 2 is dependent on the result of Job 1, Job 2 cannot begin until Job 1 finishes.

\subsubsection{Scheduling to Multiple Cores}

Here, we will discuss different scheduling algorithms. Figure 7.3 shows an overview of different scheduling algorithms. Obviously, more complicated scheduling may save more energy savings, but would potentially bring extra computation overhead.

Scheduling jobs to multiple cores can be done in multiple possible ways. Jobs can be reordered as long as the deadline is met and can be assigned to any of the multiple cores. Determining exactly how to schedule all the jobs in order to meet the deadline while also minimizing energy consumption is non-trivial. It is also important to note that the frequency being used is shared between the 


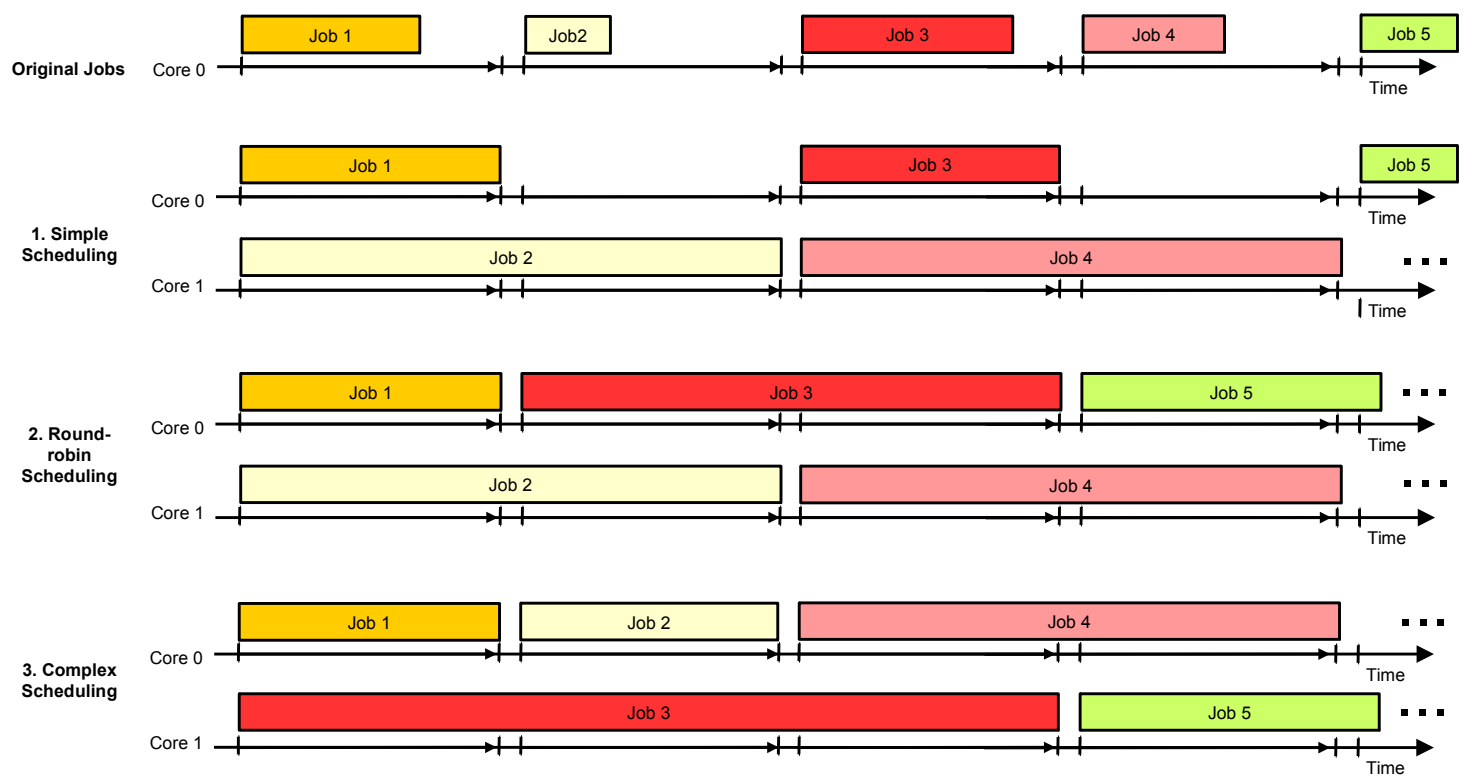

Figure 7.3: Overview of different scheduling algorithms.

cores, although it may not be true for other platforms. For the implementation, I used a naive scheduling that is simple and quick, which is shown in Schedule 1 of Figure 7.3. I simply assign the jobs to the cores in the order they come in. For example, if we have 2 cores, Job 1 will be assigned to core 1, Job 2 to core 2, Job 3 to core 1, and so on. This approach is easy to implement and quick to compute, although it may not produce an optimal ordering.

This algorithm could be improved using a round-robin scheduling as shown in Schedule 2 of Figure 7.3. This approach is very similar to the one we have already implemented. The difference from the first algorithm is that the next available job (e.g. Job 3) begins before the previous job (e.g. Job 2) ends. It will require more intelligent handling of changing frequencies and when we are able to start new jobs executing.

A better scheduling may be produced through a more intelligent algorithm, 
such as Schedule 3 in Figure 7.3. This schedule takes multiple future jobs into account during scheduling. It notices that the Job 3 is very long with very little slack time and instead of waiting for Job 1 and 2 to be scheduled, Job 3 gets scheduled as soon as possible on core 2. This allows for much more energy savings since Job 3 no longer has to run close to maximum frequency. Since Job 2 is very short, this schedule still allows it to run at reduced frequency and meet its deadline.

This kind of intelligent schedule is difficult to design due to the limited number of jobs we have knowledge about at any time. We do not have omniscient knowledge about all the jobs in the future and are instead only able to predict a few jobs ahead of time. Another issue with more complicated schemes is that in order to take predicted execution time into account during scheduling, the program slicing must occur prior to scheduling which takes time. The energy savings from an intelligent and efficient scheduler could potentially offset the its computational overhead.

\subsubsection{Evaluation}

Experimental setup is almost the same as the rest of the thesis except that I add new scheme parallel. The scheme prediction is the same as offline in the thesis.

- prediction: This is the prediction-based controller on single-core systems described in the original thesis. I use an off-line profiling.

- parallel: This is the prediction-based controller on multi-core systems described in this section. In this study, I show the results with two cores. 

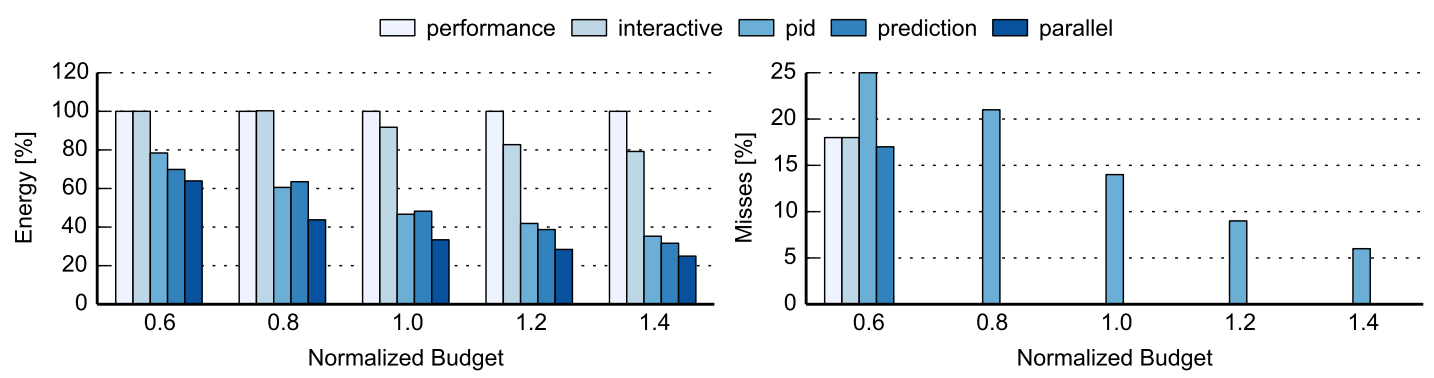

Figure 7.4: Normalized energy usage and deadline misses with two A7 (little) cores for sha.
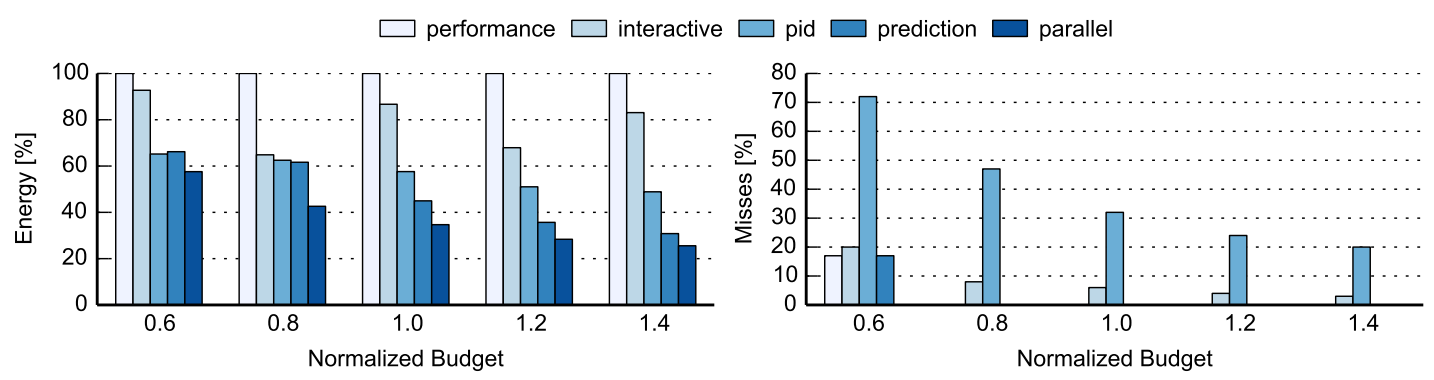

Figure 7.5: Normalized energy usage and deadline misses with two A15 (big) cores for sha.

I have the result from one application sha from the MiBench suite [25]. Although I have not added more benchmarks, I believe that general trends of the result would be similar. For the future, I plan to add more complicated benchmark evaluation results.

Figure 7.4 shows energy and deadline misses for different controllers on A7 core (little core). I swept the time budget from $60 \%$ to $140 \%$. That is, each group of bars indicates a time budget of $30,40,50,60$, and $70 \mathrm{~ms}$, respectively. I normalized energy usage to the performance governor. The deadline misses are reported as relative percentage of jobs that miss their response time requirements. For multi-core experiments, I use two little cores or two big cores. Although I do not have the result with more cores, I expect that using more cores can save more energy, but would bring more computational and communication over- 
head as well.

We see, on average, that the prediction-based controller on multi-core outperforms all other controllers in terms of both energy savings and deadline misses. On normalized budget of 1.0, the prediction-based controller with multi-cores saves $76.6 \%$ energy compared to the performance governor running at max frequency all the time. This is $58.35 \%$ more savings than the interactive governor and $13.26 \%$ more savings than the PID-based controller. Also, this is $14.82 \%$ more savings than the same prediction-based controller but with single core. This saving is from the fact that we can decrease the frequency even more by scheduling the jobs to different cores.

On normalized budget of 0.6 , all other governors except the controller with multi-cores show many deadline misses. The number of misses, however, is typically close to the number seen with the performance governor. This implies that most of the deadline misses are ones that are impossible to meet the specified time budget even with running at the maximum frequency. The prediction-based controller with multi-cores, however, is able to achieve no deadline misses by scheduling longer job to different core beforehand.

Overall, we see that the interactive governor (interactive) has a small number of deadline misses, but consumes more energy. The PID-based controller ( $\mathrm{pid}$ ) shows low energy consumption, but shows many deadline misses because it cannot follow sudden changes in job-to-job execution time. The prediction-based governor with single-core (prediction) shows low deadline misses and high energy savings, but the scheme with multi-cores (parallel) shows even more energy savings. More importantly, this scheme is able to lower the deadline misses even when the performance governor (performance) has 
some deadline misses due to tighter budget.

I also show the evaluation results on A15 core (big core) in Figure 7.4 Note that I set a default time budget as $20 \mathrm{~ms}$ because a big core is more powerful. In brief, we can see the similar trends with experiments on little cores.

\subsubsection{Limitation}

In this section, we explored the prediction-based DVFS controller on multi-core systems. It predicts the appropriate frequency and schedules the job accordingly to just meet the deadline. Compared to single-core system, this approach with multi-cores is able to decrease the frequency even more by executing the job in parallel on multiple cores. The results show $76.6 \%$ energy savings compared to the performance governor running at highest frequency. Moreover, this approach achieves no deadline misses even with a tighter time budget by scheduling a longer job earlier.

There are, however, some limitations in this extension. First, we assume that we can predict the input for future jobs to predict the execution time for multiple jobs beforehand. This is possible for decoders or streaming applications. This can be, however, challenging for some applications including interactive games or web browsers, which involve unpredictable user interactions at run-time.

Second, we assume that there is no dependency between jobs, but this may not be true for some applications. For the future research, we would like to focus on more optimized and smarter scheduling with more cores with a consideration of such limitations. 


\subsection{Multi-job DVFS}

In the thesis, we set the DVFS level for single job only (Single-job DVFS). In this section, we will discuss potential benefits by setting the DVFS level across multiple jobs (Multi-job DVFS). Compared to the single-job DVFS scheme, the multi-job DVFS scheme obtained energy savings of around $8 \%$ with no deadline misses. More importantly, running with the multi-job DVFS scheme can effectively remove deadline misses when the time budget is shorter.

\subsubsection{Motivational Example}

(a) No DVFS

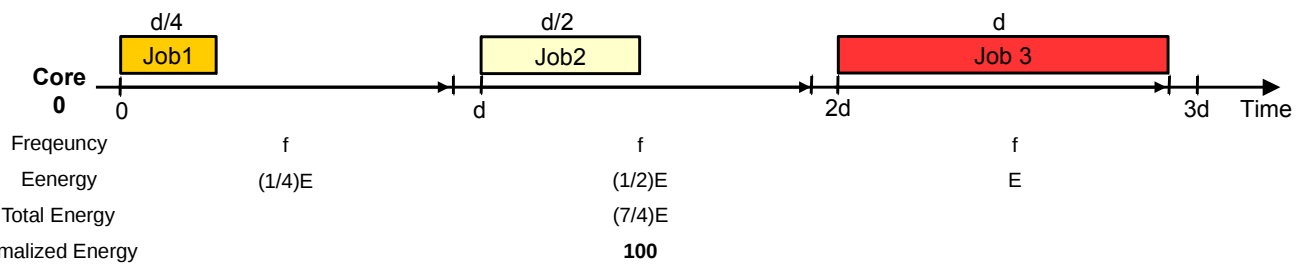

(b) Single-job

DVFS

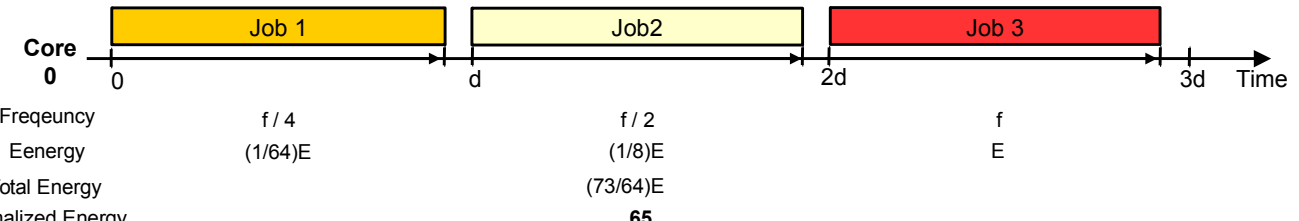

Normalized Energy (c) Multi-job
DVFS

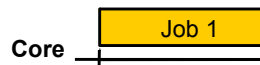

65$$
\text { ( }
$$

Freqeuncy

Eenergy (Core 0) (49/576)E

Total Energy

Normalized Energy

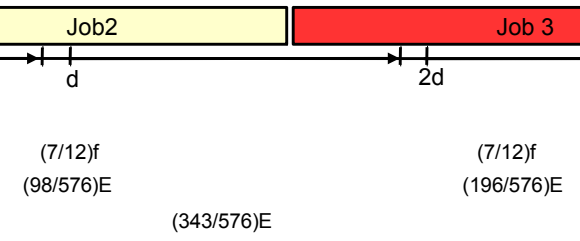

34

Figure 7.6: Motivational example ((a) no DVFS : This is run at maximum frequency $f$, (b) Single-job DVFS : This scales the frequency within a single job, and (c) Multi-job DVFS : This scales the frequency across multiple jobs).

Here, I introduce a motivational example to show how multi-job DVFS ap- 
proach can save more energy compared to single-job DVFS case [10] in Figure 7.6. Note that previous work [39] motivated this extension.

In this example, we refer to $f$ as the maximum frequency, $d$ as a time budget (i.e. deadline time), and $E$ as a normalized energy for the job which takes $d$ time to finish at frequency $f$. We also use the following equations to calculate approximate energy for each job:

$$
\begin{gathered}
E n=P t \\
P \propto V^{2} F, \quad V \propto F, \quad P \propto F^{3}
\end{gathered}
$$

where $E n$ is an energy, $P$ is a power, $V$ is a voltage, and $F$ is frequency.

First, case (a) shows original job sequences with no DVFS. Essentially, this represents the Linux Performance governor running at maximum frequency all the time. Let us assume that Job 1, 2 and 3 takes $d / 4, d / 2$, and $d$ time, respectively. As all the jobs run at the highest frequency $f$, total energy is $(7 / 4) E$. Second, case (b) shows when setting the frequency to just meet the deadline. We assume that the execution time is linearly proportional to the frequency. For example, Job 2 runs $2 x$ longer when the frequency $f$ becomes in half. As we can decrease the frequency, the energy consumption decreases to (73/64)E. Finally, case (c) shows when setting the frequency across the multiple jobs. As we can schedule Job 2 earlier, the frequency can decrease even further, which results in more energy savings $((31 / 64) E)$. In brief, if we normalize the total energy to case (a), multi-job DVFS approach (case (c)) can save 65\% energy more than the case (a), and this is $46 \%$ energy savings compared to the case (b). Although, there can be idle energy and some overhead such as setting new DVFS level, we argue that the energy savings by decreasing the frequency can outweigh such overheads. This further energy savings mainly motivate this extension. 

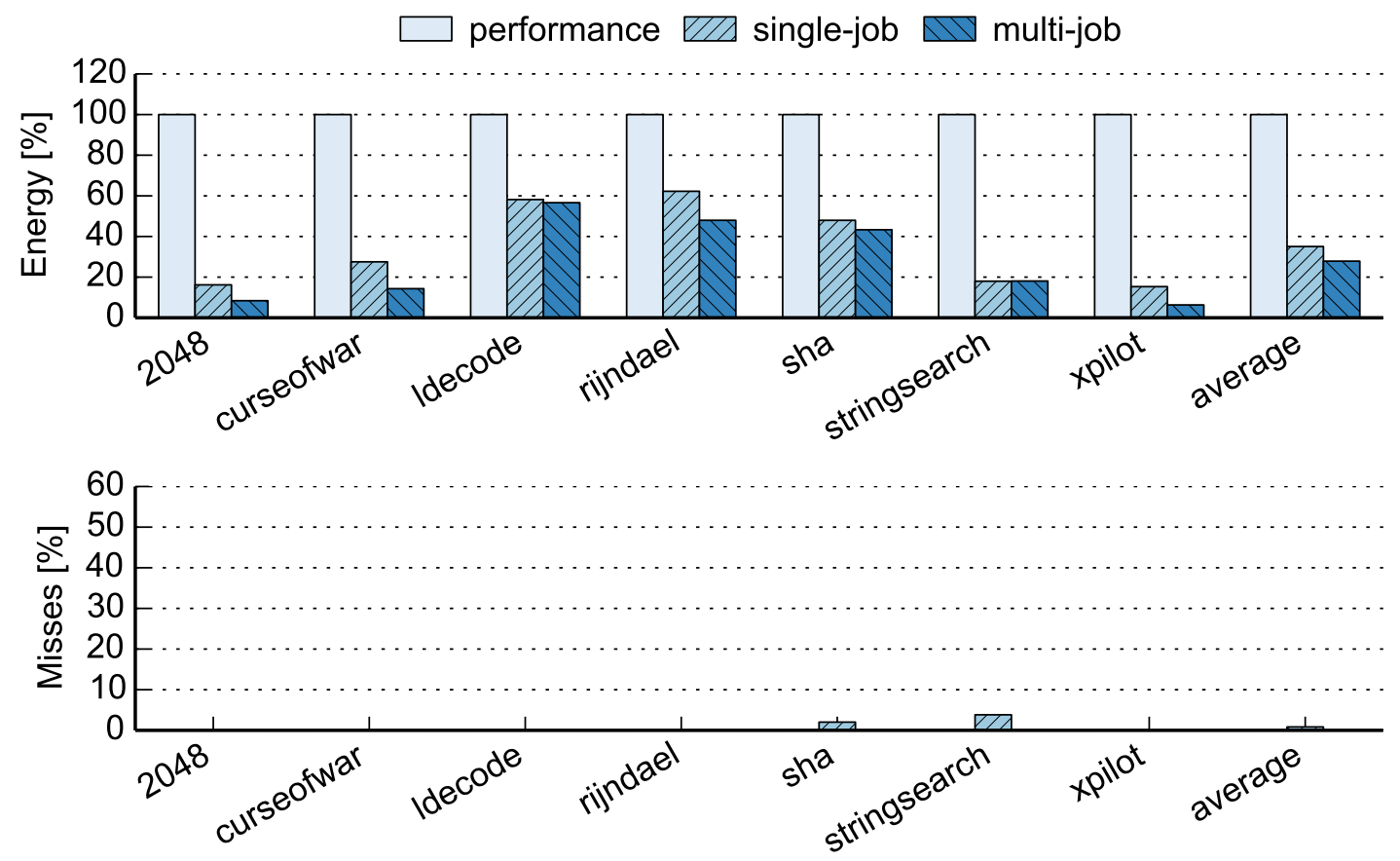

Figure 7.7: Normalized energy usage and deadline misses with single-job and multi-job DVFS scheme.

\subsubsection{Evaluation}

Experimental setup is almost the same as the rest of the thesis except that I add new scheme multi-job. The scheme single-job is the same as offline in the thesis.

- single-job: This is the prediction-based controller described in the thesis. This only sets the DVFS level for a single job. I use an off-line profiling.

- multi-job: This is the prediction-based controller which sets the DVFS level across multiple jobs. I use the off-line profiling.

Figure 7.7 shows energy and deadline misses for the various benchmarks on A7 core (little core). The experimental results show the benefit of the multi-job 

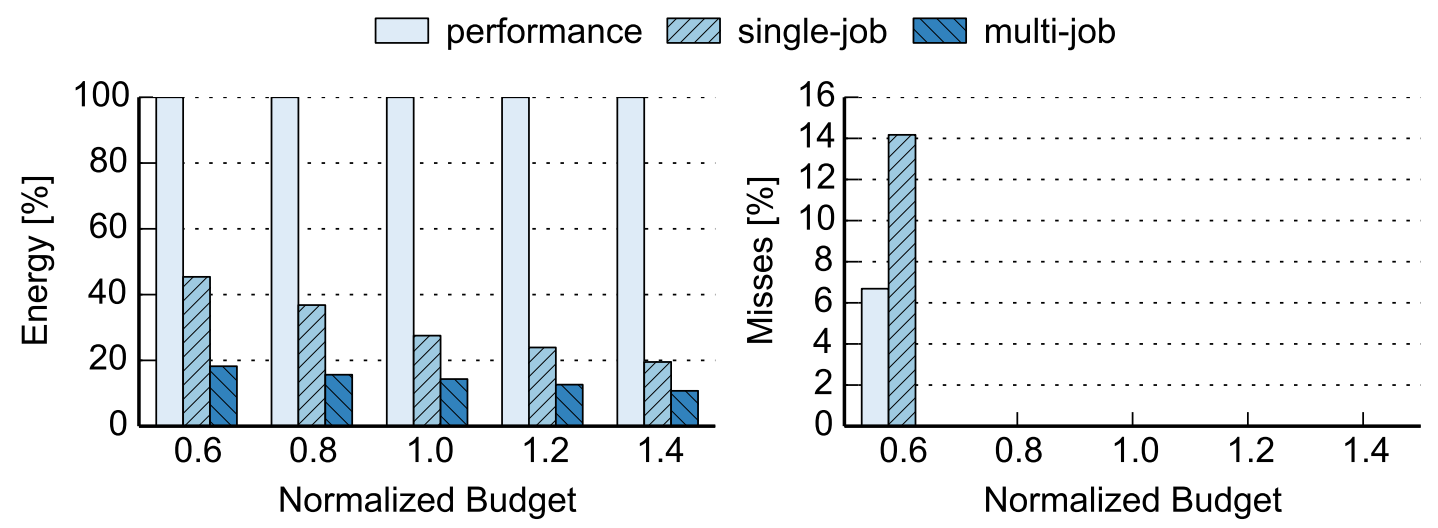

Figure 7.8: Normalized energy usage and deadline misses as time budget is varied (curseofwar).

DVFS scheme. On average, multi-job DVFS scheme achieves $8 \%$ energy savings over single-job DVFS scheme with no deadline misses. For some benchmarks (ldecode and stringsearch), there is only small energy savings. This is due to the fact that DVFS level is already similar across the jobs, so there is no difference between single-job and multi-job DVFS schemes.

Figure 7.8 shows energy and deadline misses as the normalized budget is swept below and above 1 (curseofwar). On normalized budgets of 0.6, all other governors except the multi-job DVFS scheme show many deadline misses. Even for the performance governor, there are many deadline misses. This implies that most of the deadline misses are ones that are impossible to meet at the specified time budget, even with running at the maximum frequency. The multi-job DVFS scheme, however, is able to achieve no deadline misses by borrowing some time budget from a shorter job. That is, the shorter jobs can finish before their original deadlines, and the longer jobs can start early. 


\subsubsection{Limitation}

In this section, I explored the multi-job DVFS scheme. It predicts the appropriate frequency across multiple jobs in order to achieve further energy savings and effectively remove the deadline misses with a tighter response-time requirement. Compared to the single-job DVFS scheme, this approach is able to decrease the frequency even more by executing the job at the same frequency for multiple jobs. The results show $8 \%$ energy savings compared to single-job DVFS scheme, and $72.2 \%$ energy savings over the performance governor running at highest frequency. Moreover, this approach achieves no deadline misses even with a tighter time budget by borrowing the time budget from shorter jobs.

Similar to the previous extension (Section 7.1), the multi-job DVFS scheme has some limitations. First, we assume that we can predict the input for future jobs to predict the execution time for multiple jobs beforehand. This is possible for decoders or streaming applications. This can be, however, challenging for some applications including interactive games or web browsers, which involve unpredictable user interactions at run-time. Seconds, there can be additional memory overhead. This is because we save the output for the jobs which finish earlier than the given deadline. As an examples in video decoders, if a frame is

decoded earlier than the given deadline, we need to hold the result in memory in order to decode the next frame. For the future, I would like to study how to extend this work to general applications and how to handle such memory overhead. 


\subsection{Multi-threaded Applications}

In the thesis, we have studied only running single-threaded application at a time. Extending this work to multi-threaded may require another way to model and estimate the contention of multiple threads or workloads. In this section, we will discuss some challenges in predicting the execution time for multi-threaded applications and show preliminary results.

\subsubsection{Execution Time Prediction of Multiple Threads}

As multi-threaded applications become more widely used, it is important to predict the accurate execution time not only for single-threaded applications, but also for multi-threaded applications. At a high-level, we need to estimate the longest execution time among multiple threads in order to meet the deadline. In order to find the longest execution time, we can predict the execution time for each thread and compare them. It is, however, not straightforward to estimate the longest execution time from different threads, because we need to carefully figure out and understand how each thread works to instrument the code of each thread. If some threads share the code and some does not, or if some threads depends on each other, the problem becomes more complicated.

In addition, naively applying the prediction approach described in the thesis may not work due to the fact that the execution time for different threads can vary significantly. For example, one thread may be blocked by other threads due to shared resources. In this case, the execution time may become longer than expected. Also, the execution time for different threads can vary because 
of their different roles. For instance, one thread keeps getting inputs from users, while another thread does the computation only.

Also, the more threads we have, the higher slicing overhead we would have. This can be a significant problem in practice, especially for modern applications which support an increasing number of threads.

\subsubsection{Evaluation}

Experimental setup is almost the same as the rest of the thesis except that we use one benchmark (Water-Nsquared) from Splash2 [40], which is one of well-known multi-threaded benchmark suites. The application, Water-Nsquared, spans $N$ threads, and each thread computes forces and potentials in water molecules systems. Then, it accumulated the result from each thread at the end. Although I did not evaluate this extension thoroughly with many benchmarks, I believe that the result I show here can give at least some preliminary pictures of how well the DVFS controller with on-line training works for simple multi-threaded applications.

The benchmark suite supports the flexibility of different $N$, so that we can run the same workload with different number of threads. I also ran the benchmark by pinning it on specific cores in order to test with different number of cores. More specifically, I ran the benchmark with five different configurations:

- $\mathrm{t} 1$ : One thread on once core (Figure 7.9).

- t4-c1 : Four threads on one core (First graph in Figure 7.10).

- t4-c2 : Four threads on two cores (Second graph in Figure 7.10). 


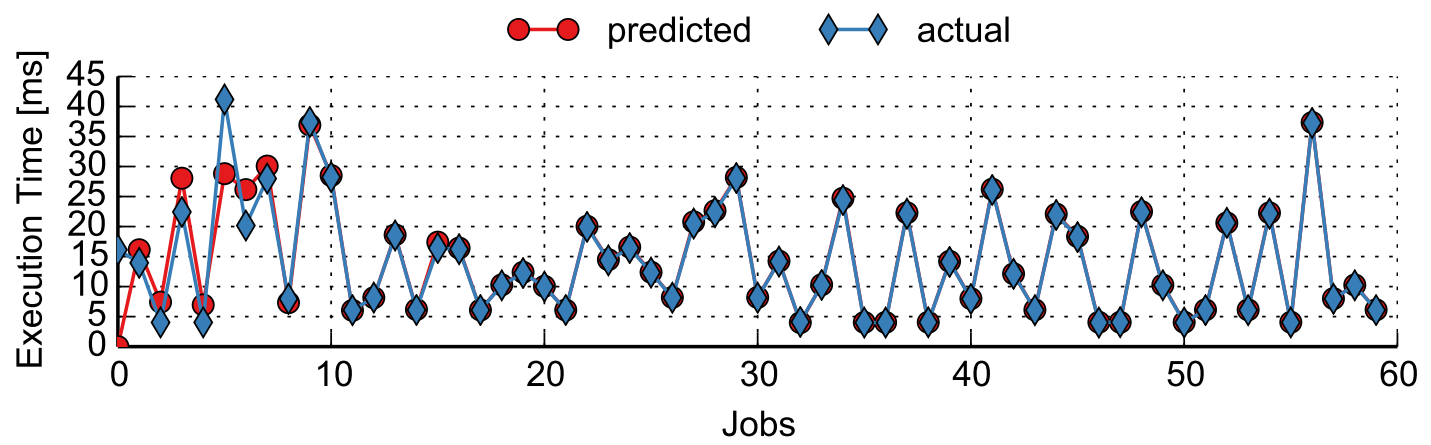

Figure 7.9: Predicted time and actual time with one thread on one core (Water-Nsquared).

- t4-c3 : Four threads on three cores (Third graph in Figure 7.10).

- t4-c4 : Four threads on four cores (Fourth graph in Figure 7.10).

At first, I used all the program features from program slice of $N$ threads, but it turned out the set of feature values from different threads is very similar to each other. To minimize the slicing overhead, I only use the program features from the main thread (thread 0 ) and train the model only with the features from thread 0 .

Figure 7.9 and Figure 7.10 shows the predicted time and actual time with a different number of threads and cores. From $t 4-\mathrm{c} 1$ and $t 1$, we can see that using more threads does not give much benefits if we have only one core. As we use more cores (from $t 4-\mathrm{c} 1$ to $t 4-\mathrm{c} 4$ ), we can see that the execution time decreases. This is because multiple threads can be executed on different cores concurrently.

Figure 7.11 shows the prediction errors with five different configurations. In general, the on-line training scheme on simple multi-threaded applications give a reasonable prediction accuracy. I exclude the data during initial phase to show 

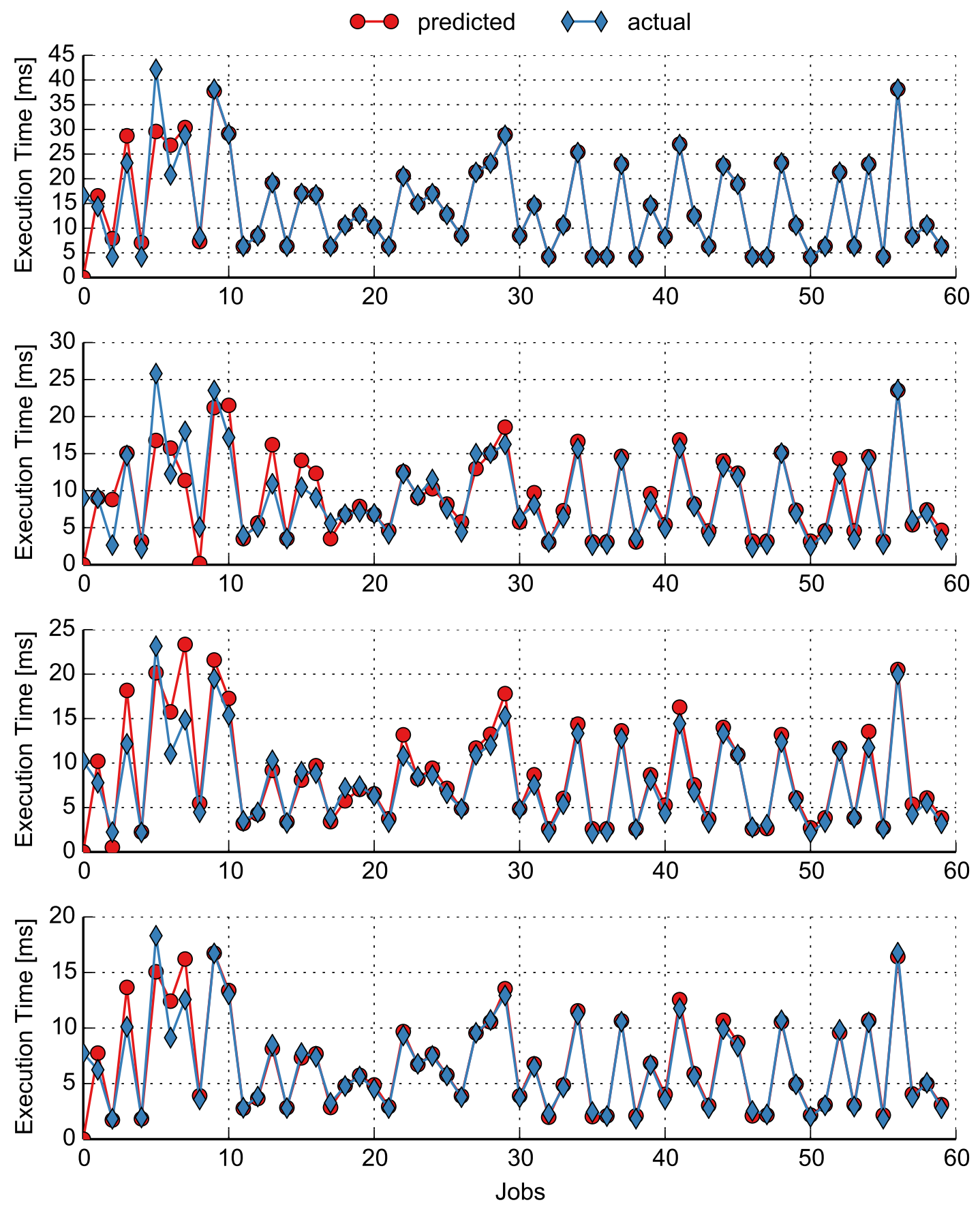

Figure 7.10: Predicted time and actual time with four threads on different number of cores. From the top, the number of cores is 1, 2, 3, and 4, respectively (Water-Nsquared). 


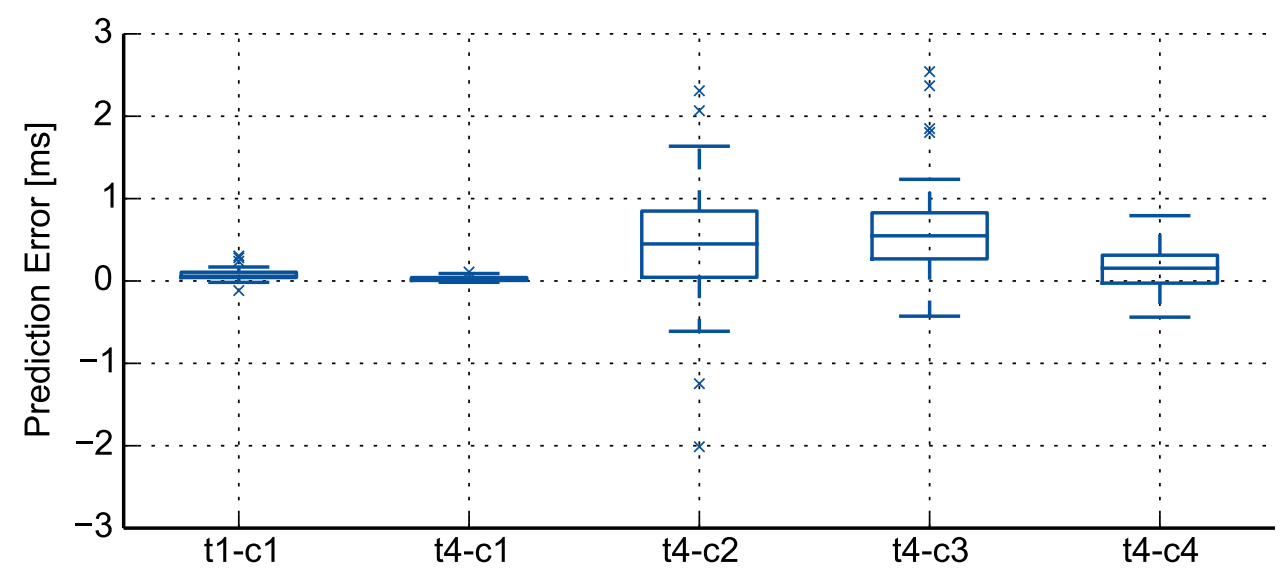

Figure 7.11: Prediction errors as the number of threads and cores is varied (Water-Nsquared).

the errors after the model becomes stable. For all the configurations with on-line training, we can see prediction errors of less than $5 \mathrm{~ms}$, which is only $10 \%$ of a $50 m s$ time budget.

This result also shows another benefit of my on-line learning capability. Since the execution time with different number of threads and cores may vary, if we use the off-line training model, we need to train the model for every other configurations. The on-line training model, however, can be easily used even for different configurations without any manual efforts at off-line.

I believe that using the program features only from the main thread works because each thread has a similar amount of workload. In other words, since the job is almost equally distributed to each thread, the execution time of each thread is roughly same. 


\subsubsection{Limitation}

In this section, we looked at possible extensions to multi-threaded applications with a preliminary result. As a simple way, we predict the execution time of multi-threaded workload by applying the program slicing technique to main thread. The preliminary result shows that the prediction-based DVFS controller with on-line training scheme works reasonably well for simple applications. Without any modification, the on-line training scheme can predict the different execution time for different number of threads and cores.

This approach, however, may not be practical for general multi-threaded applications. The simple application I tested distributes the workload to each thread equally, so the execution time of each thread is similar. Also, each thread can be run independently for most of times. It is, therefore, relatively easy to predict when all threads finish by estimating the execution time from single thread. The execution time for different threads, however, actually can vary significantly due to many reasons, such as the synchronization delay between multiple threads or different roles of each thread. For instance, one thread may wait for another thread to finish due to some shared resources, which results in longer execution time for such threads. As an another example, one thread can

only take care of getting inputs from users, while another thread can only do the heavy computation. In these cases, the simple approach such as considering the program features only from the main thread may not work. As part of the future work, I would like to study how to extend this work to general and more complicated multi-threaded applications. 


\section{CHAPTER 8}

\section{CONCLUSION}

In this thesis, I present a prediction-guided DVFS and migration controller for interactive applications, which can continuously adapt to operating environments at run-time. The controller predicts the execution time of a job, and sets the DVFS level and chooses the best core to use in order to reduce energy consumption without violating response-time requirements. Experimental results show that the new on-line training approach enables the controller to avoid deadline misses even when unexpected interference is added a run-time, and to be easily applied for diverse platforms without customized off-line training. The results also suggest that this prediction-guided controller provides significant energy savings over traditional Linux power governors. 


\section{APPENDIX A \\ SOLVING LEAST SQUARES APPROXIMATION}

The unknown parameters of the linear model can be found using the linear least square approximation. There are multiple methods to solve the least squares approximation problem. We will discuss how to derive these methods in more detail.

\section{A.1 Linear Least Squares Approximation}

As we discussed in Chapter 4.2, let us define our linear model as

$$
\bar{y}=x \beta
$$

where $\bar{y}$ is the predicted execution time, $x$ is a vector of feature values, and $\beta$ are the coefficients that map feature values to execution time. Table A.1 summarizes the variables and notation used in this appendix.

The linear least squares approximation finds the optimal coefficients, $\boldsymbol{\beta}$,

\begin{tabular}{|c|l|l|}
\hline Variable & Type & Description \\
\hline \hline $\bar{y}$ & Scalar & Predicted execution time \\
\hline$x$ & Vector & Feature values \\
\hline $\boldsymbol{\beta}$ & Vector & Model coefficients \\
\hline \hline$y$ & Vector & A set of actual execution times \\
\hline$X$ & Matrix & A set of generated feature values \\
\hline$X \boldsymbol{\beta}-y$ & Vector & Prediction errors \\
\hline$\|\cdot\|$ & Scalar & L2-norm (sum of squares) \\
\hline
\end{tabular}

Table A.1: Variable and notation descriptions. 
which minimize the mean square error:

$$
\underset{\beta}{\operatorname{argmin}}\|X \beta-y\|^{2}
$$

There are multiple methods to solve linear least square problem.

1. Solving ordinary least squares equation.

2. Using QR decomposition.

\section{A.2 Ordinary Least Square Equation}

As a simple method, we can solve the approximation problem A.1 by using ordinary least squares equation. The $\boldsymbol{\beta}$ that minimizes this can be calculated as

$$
\boldsymbol{\beta}=\left(X^{T} X\right)^{-1} X^{T} y
$$

\section{A.3 QR Decomposition}

As an another method to solve the least squares approximation problem, we can use a QR decomposition method. We decompose the $X$ matrix as

$$
X=Q R
$$

where $Q$ is an orthogonal matrix $\left(Q^{T}=Q^{-1}\right)$ and $R$ is an upper triangular matrix. By plug this decomposition into Equation (A.2), we can derive:

$$
R \beta=Q^{T} y
$$


More detailed steps can be described as

$$
\begin{aligned}
\boldsymbol{\beta} & =\left(X^{T} X\right)^{-1} X^{T} y \\
& =\left((Q R)^{T}(Q R)\right)^{-1}(Q R)^{T} y \\
& =\left(R^{T} Q^{T} Q R\right)^{-1}(Q R)^{T} y \\
& =\left(R^{T} Q^{-1} Q R\right)^{-1}(Q R)^{T} y \quad\left(\because Q^{T}=Q^{-1}\right) \\
& =\left(R^{T} R\right)^{-1}(Q R)^{T} y \\
& =R^{-1} R^{-T} R^{T} Q^{T} y \\
& =R^{-1} Q^{T} y \\
\therefore R \boldsymbol{\beta} & =Q^{T} y
\end{aligned}
$$

Note that the matrix $Q$ itself is not necessary to obtain $\beta$ as long as $d$ is available:

$$
R \boldsymbol{\beta}=Q^{T} y=d
$$

Due to the property of upper-triangular matrix, the coefficients $\beta$ can be easily computed. The challenge now becomes how to quickly calculate $R$ and $d$.

For fast training, we keep the intermediate result of $R$ and $d$, and directly update them with a new training sample from the most recent job by adding a row and applying a series of Givens rotations $G=G_{1} G_{2} \ldots G_{k}$. The Givens rotation is a well-known matrix transformation which introduces zeros in matrices: 


$$
G(i, j, \theta)=\left[\begin{array}{ccccccc}
1 & \ldots & 0 & \ldots & 0 & \ldots & 0 \\
\vdots & \ddots & \vdots & & \vdots & & \vdots \\
0 & \ldots & c & \ldots & s & \ldots & 0 \\
\vdots & & \vdots & \ddots & \vdots & & \vdots \\
0 & \ldots & -s & \ldots & c & \ldots & 0 \\
\vdots & & \vdots & & \vdots & \ddots & \vdots \\
0 & \ldots & 0 & \ldots & 0 & \ldots & 1
\end{array}\right]
$$

where $c=\cos \theta$ and $s=\sin \theta$ at the $i$ th and $j$ th rows and columns and $c$ and $s$ is determined by the element at the $i$ th and $j$ th rows.

Multiplying $G(i, j, \theta)$ to the $X$ matrix rotates it in the $(i, j)$ plane of $\theta$ radians in counterclockwise. The Givens rotation can be used for computing the $\mathrm{QR}$ decomposition. In this work, we apply a series of Givens rotations to calculate new matrix $R$ and $d$. Next, we will discuss how to calculate them with a simple example. 
APPENDIX B

EXAMPLE OF FAST UPDATES USING QR DECOMPOSITION

The linear model with growing samples can be quickly updated using QR decomposition method. To give more concrete ideas, we will see a simple example of how to find and update the model.

As an example, we will use the same linear least squares approximation problem (describe in Appendix A). Here, we need to find the optimal coefficients, $\boldsymbol{\beta}$, which minimize the mean square error:

$$
\underset{\beta}{\operatorname{argmin}}\|X \boldsymbol{\beta}-y\|^{2}
$$

For simplicity, let us assume that we have 2 features:

$$
\boldsymbol{\beta}=\left[\begin{array}{l}
\beta_{1} \\
\beta_{2}
\end{array}\right]
$$

\section{B.1 Find the Model Coefficients}

During the initial phase, assume that we have the following data ( 3 jobs with 2 features):

$$
X=\left[\begin{array}{ll}
1 & 1 \\
1 & 2 \\
1 & 3
\end{array}\right], \quad y=\left[\begin{array}{l}
2 \\
3 \\
5
\end{array}\right]
$$

The goal is to find the coefficients $\beta_{1}$ and $\beta_{2}$. Let us solve with two different methods and compare the results.

1. Solving ordinary least squares equation. 
2. Using $\mathrm{QR}$ decomposition.

\section{B.1.1 Ordinary Least Square Equation}

We can simply use Equation A.2):

$$
\boldsymbol{\beta}=\left(X^{T} X\right)^{-1} X^{T} y
$$

Let us calculate $X^{T} X$ first.

$$
X^{T} X=\left[\begin{array}{lll}
1 & 1 & 1 \\
1 & 2 & 3
\end{array}\right]\left[\begin{array}{ll}
1 & 1 \\
1 & 2 \\
1 & 3
\end{array}\right]=\left[\begin{array}{cc}
3 & 6 \\
6 & 14
\end{array}\right]
$$

We then invert the matrix $X^{T} X$.

$$
\left(X^{T} X\right)^{-1}=\left[\begin{array}{cc}
7 / 3 & -1 \\
-1 & 1 / 2
\end{array}\right]
$$

Next, we calculate the matrix $X^{T} y$.

$$
X^{T} y=\left[\begin{array}{lll}
1 & 1 & 1 \\
1 & 2 & 3
\end{array}\right]\left[\begin{array}{l}
2 \\
3 \\
5
\end{array}\right]=\left[\begin{array}{l}
10 \\
23
\end{array}\right]
$$

Finally, we plug them into Equation (A.2) to get two coefficients.

$$
\begin{gathered}
\left(X^{T} X\right)^{-1} X^{T} y=\left[\begin{array}{cc}
7 / 3 & -1 \\
-1 & 1 / 2
\end{array}\right]\left[\begin{array}{l}
10 \\
23
\end{array}\right]=\left[\begin{array}{c}
1 / 3 \\
3 / 2
\end{array}\right] \\
\therefore \beta_{1}=1 / 3, \quad \beta_{2}=3 / 2
\end{gathered}
$$




\section{B.1.2 QR Decomposition}

Recall that we have the following linear model:

$$
X \boldsymbol{\beta}=y
$$

where $y$ is the predicted value, $X$ is the feature values, and $\boldsymbol{\beta}$ is the model coefficients.

To decompose the $X$ matrix, we can apply a series of Givens rotations to both sides in Equation (B.1):

$$
\begin{gathered}
X \boldsymbol{\beta}=y \\
G X \boldsymbol{\beta}=G y
\end{gathered}
$$

where $G=G_{1} G_{2} \ldots G_{k}$ is a series of Givens rotations. From the Equation A.3, we can know $R=G X$ and $Q^{T}=G\left(\right.$ or $\left.Q=G^{T}\right)$.

To apply a series of Givens rotations to both sides in Equation (B.1), let us first merge the $X$ matrix and $y$ into one matrix $A$ as

$$
A=X \mid y=\left[\begin{array}{lll}
1 & 1 & 2 \\
1 & 2 & 3 \\
1 & 3 & 5
\end{array}\right]
$$

To introduce zeros in this matrix, we apply a series of Givens rotations. This eventually generates the $R$ matrix and $d\left(=Q^{T} y\right)$ in Equation (A.3). First Givens rotation is determined by the element of the matrix $A$ :

$$
G_{3,1}=\left[\begin{array}{ccc}
1 & 0 & 0 \\
0 & 0.707 & 0.707 \\
0 & -0.707 & 0.707
\end{array}\right]
$$


Then, the next Givens rotation is determined by intermediate result $G_{3,1} A$ :

$$
G_{2,1}=\left[\begin{array}{ccc}
0.577 & 0.816 & 0 \\
-0.816 & 0.577 & 0 \\
0 & 0 & 1
\end{array}\right]
$$

Similarly, the last one is determined by $G_{2,1} G_{3,1} A$ :

$$
G_{3,2}=\left[\begin{array}{ccc}
1 & 0 & 0 \\
0 & 0.866 & 0.5 \\
0 & -0.5 & 0.866
\end{array}\right]
$$

By applying these rotations into the $A$ matrix:

$$
G_{3,2} G_{2,1} G_{3,1} A=\left[\begin{array}{ccc}
1.732 & 3.464 & 5.774 \\
0 & 1.414 & 2.121 \\
0 & 0 & 0.408
\end{array}\right]
$$

Finally, this can be represented as the $R$ matrix and $d$. As shown, $R$ is the uppertriangular matrix.

$$
R=\left[\begin{array}{cc}
1.732 & 3.464 \\
0 & 1.414 \\
0 & 0
\end{array}\right], \quad d=\left[\begin{array}{l}
5.774 \\
2.121 \\
0.408
\end{array}\right]
$$

From Equation (A.3),

$$
\begin{gathered}
R \boldsymbol{\beta}=d \\
{\left[\begin{array}{cc}
1.732 & 3.464 \\
0 & 1.414 \\
0 & 0
\end{array}\right]\left[\begin{array}{l}
\beta_{1} \\
\beta_{2}
\end{array}\right]=\left[\begin{array}{l}
5.774 \\
2.121 \\
0.408
\end{array}\right]}
\end{gathered}
$$

Recall that due to the property of upper-triangular matrix, the coefficients $\beta$ can 
be easily computed.

$$
\begin{aligned}
\beta_{2} & =2.121 / 1.414=3 / 2 \\
\beta_{1} & =\left(5.775-3.464 \times \beta_{2}\right) / 1.732 \\
& =(5.775-3.464 \times 3 / 2) / 1.732 \\
& =1 / 3 \\
\therefore \beta_{1} & =1 / 3, \quad \beta_{2}=3 / 2
\end{aligned}
$$

From the final result, we can verify that the coefficients are the exactly same from two different methods.

\section{B.2 Update the Model with New Samples}

I have shown the example of how to find the model coefficients with two different methods. The more important problem is how to update the model coefficients when new sample is added to the original matrix. Let us assume that we have a new data:

$$
x_{\text {new }}=\left[\begin{array}{ll}
1 & 4
\end{array}\right], \quad y_{\text {new }}=[8]
$$

where $x_{\text {new }}$ and $y_{\text {new }}$ represent a new sample. By adding this to previous $X$ and $y$, we can get the following matrix:

$$
\tilde{X}=\left[\begin{array}{ll}
1 & 1 \\
1 & 2 \\
1 & 3 \\
1 & 4
\end{array}\right], \quad \tilde{y}=\left[\begin{array}{l}
2 \\
3 \\
5 \\
8
\end{array}\right]
$$

where $\tilde{X}$ and $\tilde{y}$ represent updated $X$ and $y$. 
The goal is to update the coefficients $\beta_{1}$ and $\beta_{2}$ with the updated data. Similarly, let us solve with two different methods and compare the results.

1. Solving ordinary least squares equation.

2. Using $Q R$ decomposition.

\section{B.2.1 Ordinary Least Square Equation}

In a naive approach, we can solve Equation A.2 with new data from the beginning. This naive approach brings increasing overhead as the new sample is coming. As the size of the matrix increase, computational overhead increases exponentially. Also, limited memory can be a problem to hold ever growing matrix.

A better solution is to keep the intermediate result of the least squares approximation. Instead of keeping $X$ and $y$, we can keep $X^{T} X$ and $X^{T} y$ and incrementally updated them:

$$
\begin{aligned}
\tilde{X}^{T} \tilde{X} & =X^{T} X+x_{\text {new }}{ }^{T} x_{\text {new }} \\
\tilde{X}^{T} \tilde{y} & =X^{T} y+x_{\text {new }}{ }^{T} y_{\text {new }}
\end{aligned}
$$

This approach enables keeping track of a large number of training samples with a fixed memory footprint.

From the previous $X^{T} X$ and $x_{\text {new }}$,

$$
X^{T} X=\left[\begin{array}{cc}
3 & 6 \\
6 & 14
\end{array}\right], \quad x_{\text {new }}=\left[\begin{array}{ll}
1 & 4
\end{array}\right]
$$


we can update new $\tilde{X}^{T} \tilde{X}$ as

$$
\tilde{X}^{T} \tilde{X}=\left[\begin{array}{cc}
3 & 6 \\
6 & 14
\end{array}\right]+\left[\begin{array}{l}
1 \\
4
\end{array}\right]\left[\begin{array}{ll}
1 & 4
\end{array}\right]=\left[\begin{array}{cc}
3+1 \times 1 & 6+1 \times 4 \\
6+4 \times 1 & 14+4 \times 4
\end{array}\right]=\left[\begin{array}{cc}
4 & 10 \\
10 & 30
\end{array}\right]
$$

Similarly, from the previous $X^{T} y, x_{\text {new }}$, and $y_{\text {new }}$,

$$
X^{T} y=\left[\begin{array}{l}
10 \\
23
\end{array}\right], \quad x_{\text {new }}=\left[\begin{array}{ll}
1 & 4
\end{array}\right], \quad y_{\text {new }}=[8]
$$

we can update new $\tilde{X}^{T} \tilde{y}$ as

$$
\tilde{X}^{T} \tilde{y}=\left[\begin{array}{l}
10 \\
23
\end{array}\right]+\left[\begin{array}{l}
1 \\
4
\end{array}\right][8]=\left[\begin{array}{l}
10+1 \times 8 \\
23+4 \times 8
\end{array}\right]=\left[\begin{array}{l}
18 \\
55
\end{array}\right]
$$

Note that the size of the two matrices $X^{T} X$ and $X^{T} y$ does not increase with new data. That is, it depends only on the size of $\boldsymbol{\beta}$ and not the size of training set (the number of rows in $X$ ). For example, for $k$ features, the size of $X^{T} X$ and $X^{T} y$ are fixed as $k \times k$ and $k \times 1$. In this example, the size is $2 \times 2$ and $2 \times 1$, relatively.

After we get the two matrices $\tilde{X}^{T} \tilde{X}$ and $\tilde{X}^{T} \tilde{y}$, we can calculate the coefficients by plug them into Equation (A.2). Before this, however, we still need to invert the $\tilde{X}^{T} \tilde{X}$ matrix:

$$
\left(\tilde{X}^{T} \tilde{X}\right)^{-1}=\left[\begin{array}{cc}
3 / 2 & -1 / 2 \\
-1 / 2 & 1 / 5
\end{array}\right]
$$

Finally, we can get two coefficients:

$$
\begin{gathered}
\left(\tilde{X}^{T} \tilde{X}\right)^{-1} \tilde{X}^{T} \tilde{y}=\left[\begin{array}{cc}
3 / 2 & -1 / 2 \\
-1 / 2 & 1 / 5
\end{array}\right]\left[\begin{array}{c}
18 \\
55
\end{array}\right]=\left[\begin{array}{c}
-1 / 2 \\
2
\end{array}\right] \\
\therefore \beta_{1}=-1 / 2, \quad \beta_{2}=2
\end{gathered}
$$

Due to the matrix inversion, the computation complexity of this approach is still $\operatorname{high}\left(O\left(k^{3}\right)\right)$. 


\section{B.2.2 QR Decomposition}

When solving ordinary least squares equation, we keep the intermediate result $X^{T} X$ and $X^{T} y$. For fast training using the $\mathrm{QR}$ decomposition, we also keep the intermediate result $R$ and $d$.

Instead of decomposing the matrix $X$ again, we can directly update them with a new training samples by adding them to previous $R$ and $d$ and by applying a series of Givens rotations.

First, let us merge $R$ and $d$ into one matrix $A$ :

$$
A=R \mid d=\left[\begin{array}{ccc}
1.732 & 3.464 & 5.774 \\
0 & 1.414 & 2.121 \\
0 & 0 & 0.408
\end{array}\right]
$$

Because we have two coefficients only, the last row of the $A$ matrix is not necessary. Hence, we can remove the last row to keep the matrix constant. After removing the last row, we add the new sample $x_{\text {new }}$ and $y_{\text {new }}$ to top row.

$$
\begin{gathered}
x_{\text {new }}=\left[\begin{array}{ll}
1 & 4
\end{array}\right], \quad y_{\text {new }}=[8] \\
A^{\prime}=\left[\begin{array}{ccc}
1 & 4 & 8 \\
1.732 & 3.464 & 5.774 \\
0 & 1.414 & 2.121
\end{array}\right]
\end{gathered}
$$

To introduce zeros in this matrix, we apply a series of Givens rotations. This eventually generates the new $\tilde{R}$ matrix and $\tilde{d}$ in Equation (A.3). First Givens rotation is determined by the element of the matrix $A^{\prime}$ :

$$
G_{2,1}=\left[\begin{array}{ccc}
0.5 & 0.866 & 0 \\
-0.866 & 0.5 & 0 \\
0 & -0 & 1
\end{array}\right]
$$


Then, the next Givens rotation is determined by intermediate result $G_{2,1} A^{\prime}$ :

$$
G_{3,2}=\left[\begin{array}{ccc}
1 & 0 & 0 \\
0 & -0.755 & 0.632 \\
0 & 0.632 & 0.755
\end{array}\right]
$$

By applying these rotations into the $A^{\prime}$ matrix:

$$
G_{3,2} G_{2,1} A=\left[\begin{array}{ccc}
2 & 5 & 9 \\
0 & 2.236 & 4.041 \\
0 & 0 & 0.913
\end{array}\right]
$$

Finally, this can be represented as the new $\tilde{R}$ matrix and $\tilde{d}$. As shown, $\tilde{R}$ is also the upper-triangular matrix.

$$
\tilde{R}=\left[\begin{array}{cc}
2 & 5 \\
0 & 2.236 \\
0 & 0
\end{array}\right], \quad d=\left[\begin{array}{c}
9 \\
4.472 \\
0.913
\end{array}\right]
$$

From Equation (A.3),

$$
\begin{gathered}
R \boldsymbol{\beta}=d \\
{\left[\begin{array}{cc}
2 & 5 \\
0 & 2.236 \\
0 & 0
\end{array}\right]\left[\begin{array}{l}
\beta_{1} \\
\beta_{2}
\end{array}\right]=\left[\begin{array}{c}
9 \\
4.472 \\
0.913
\end{array}\right]}
\end{gathered}
$$

Recall that due to the property of upper-triangular matrix, the coefficients $\beta$ can be easily computed.

$$
\begin{aligned}
\beta_{2} & =4.472 / 2.236=2 \\
\beta_{1} & =\left(9-5 \times \beta_{2}\right) / 2 \\
& =(9-5 \times 2) / 2 \\
& =-1 / 2 \\
\therefore \beta_{1} & =-1 / 2, \quad \beta_{2}=2
\end{aligned}
$$


From the final result, we can also verify that the updated coefficients are the exactly same from two different methods.

Recall that after the Givens rotations, the last row of $R$ always becomes a zero vector and we can remove the row to keep the size of $R$ constant. This method based on the QR decomposition reduces the time complexity to $O(k)$ while keeping the history of all previous jobs with a fixed-size matrix. 


\section{BIBLIOGRAPHY}

[1] Maurits van der Schee. 2048.c. https://github.com/mevdschee/ 2048 .C.

[2] Yasuhiro Endo, Zheng Wang, J. Bradley Chen, and Margo Seltzer. Using Latency to Evaluate Interactive System Performance. In Proceedings of the 2nd USENIX Symposium on Operating Systems Design and Implementation, 1996.

[3] Stuart K. Card, George G. Robertson, and Jock D. Mackinlay. The Information Visualizer, an Information Workspace. In Proceedings of the SIGCHI Conference on Human Factors in Computing Systems, 1991.

[4] Robert B. Miller. Response Time in Man-computer Conversational Transactions. In Proceedings of the 1968 Fall Joint Computer Conference, Part I, 1968.

[5] Gitte Lindegaard, Gary Fernandes, Cathy Dudek, and J. Browñ. Attention Web Designers: You Have 50 Milliseconds to Make a Good First Impression! Behavior \& Information Technology, 25(2), 2006.

[6] Yuhao Zhu, Matthew Halpern, and Vijay Janapa Reddi. Event-Based Scheduling for Energy-Efficient QoS (eQoS) in Mobile Web Applications. In Proceedings of the 21st Symposium on High Performance Computer Architecture, 2015.

[7] Yan Gu and Samarjit Chakraborty. A Hybrid DVS Scheme for Interactive 3D Games. In Proceedings of the 14th Real-Time and Embedded Technology and Applications Symposium, 2008.

[8] Yuhao Zhu and Vijay Janapa Reddi. High-performance and Energyefficient Mobile Web Browsing on Big/Little Systems. In Proceedings of the 19th International Symposium on High-Performance Computer Architecture, 2013.

[9] Chang-Hong Hsu, Yunqi Zhang, Michael A. Laurenzano, David Meisner, Thomas Wenisch, Jason Mars, Lingjia Tang, and Ronald G. Dreslinski. Adrenaline: Pinpointing and Reining in Tail Queries with Quick Voltage Boosting. In Proceedings of the 21st Symposium on High Performance Computer Architecture, 2015. 
[10] Daniel Lo, Taejoon Song, and G. Edward Suh. Prediction-Guided Performance-Energy Trade-off for Interactive Applications. In Proceedings of the 48th International Symposium on Microarchitecture, 2015.

[11] Dominik Brodowski. CPU Frequency and Voltage Scaling Code in the Linux ${ }^{\mathrm{TM}}$ Kernel. https://android.googlesource.com/kernel/ common/+/a7827a2a60218b25f222b54f77ed38f57aebe08b/ Documentation/cpu-freq/governors.txt.

[12] Yan Gu and Samarjit Chakraborty. Control Theory-based DVS for Interactive 3D Games. In Proceedings of the 45th Design Automation Conference, 2008.

[13] Kihwan Choi, K. Dantu, Wei-Chung Cheng, and M. Pedram. Frame-Based Dynamic Voltage and Frequency Scaling for a MPEG Decoder. In Proceedings of the IEEE/ACM International Conference on Computer Aided Design, 2002.

[14] David Lo, Liqun Cheng, Rama Govindaraju, Luiz André Barroso, and Christos Kozyrakis. Towards Energy Proportionality for Large-scale Latency-critical Workloads. In Proceeding of the 41st International Symposium on Computer Architecture, 2014.

[15] Nachiappan Chidambaram Nachiappan, Praveen Yedlapalli, Niranjan Soundararajan, Anand Sivasubramaniam, Mahmut T. Kandemir, Ravi Iyer, and Chita R. Das. Domain Knowledge Based Energy Management in Handhelds. In Proceedings of the 21st Symposium on High Performance Computer Architecture, 2015.

[16] Fen Xie, Margaret Martonosi, and Sharad Malik. Compile-time Dynamic Voltage Scaling Settings: Opportunities and Limits. In Proceedings of the ACM SIGPLAN Conference on Programming Language Design and Implementation, 2003.

[17] Qiang Wu, V. J. Reddi, Youfeng Wu, Jin Lee, Dan Connors, David Brooks, Margaret Martonosi, and Douglas W. Clark. A Dynamic Compilation Framework for Controlling Microprocessor Energy and Performance. In Proceedings of the 38th International Symposium on Microarchitecture, 2005.

[18] Yongin Kwon, Sangmin Lee, Hayoon Yi, Donghyun Kwon, Seungjun Yang, Byung-Gon Chun, Ling Huang, Petros Maniatis, Mayur Naik, and Yunheung Paek. Mantis: Automatic Performance Prediction for Smartphone Ap- 
plications. In Proceedings of the 2013 USENIX Conference on Annual Technical Conference, 2013.

[19] Frank Tip. A Survey of Program Slicing Techniques. Journal of Programming Languages, 3(3), 1995.

[20] Robert Tibshirani. Regression Shrinkage and Selection via the Lasso. Journal of the Royal Statistical Society. Series B (Methodological), 1996.

[21] ODROID-XU3. http://www.hardkernel.com/main/products/ prdt_info.php?g_code=G140448267127.

[22] Sven Hammarling and Craig Lucas. Updating the QR Factorization and the Least Squares Problem. In MIMS Report, page 73, 2008.

[23] Michal Bernardelli. Usage of Algorithm of Fast Updates of QR Decomposition to Solution of Linear Regression Models. In Electronic International Interdisciplinary Conference, 2012.

[24] ARM. big.LITTLE Technology: The Future of Mobile. http: //www.arm.com/files/pdf/big_LITTLE_Technology_the_ Future_of_Mobile.pdf, 2013.

[25] Matthew R. Guthaus, Jeffrey S. Ringenberg, Dan Ernst, Todd M. Austin, Trevor Mudge, and Richard B. Brown. MiBench: A Free, Commercially Representative Embedded Benchmark Suite. In Proceedings of the 4th International Workshop on Workload Characterization, 2001.

[26] Alexey Nikolaev. Curse of War - Real Time Strategy Game For Linux. https://github.com/a-nikolaev/curseofwar.

[27] Karsten Sühring. H.264/AVC Software Coordingation. http://iphome. hhi.de/suehring/tml/.

[28] David Huggins-Daines, Mohit Kumar, Arthur Chan, Alan W. Black, Mosur Ravishankar, and Alex I. Rudnicky. Pocketsphinx: A Free, Real-Time Continuous Speech Recognition System for Hand-Held Devices. In Proceedings of the IEEE International Conference on Acoustics, Speech and Signal Processing, 2006.

[29] Dieter Plaetinck. Uzbl - Web Interface Tools Which Adhere to the Unix Philosophy. http://www.uzbl.org. 
[30] Bjørn Stabell, Ken Ronny Schouten, Bert Gÿsbers, and Dick Balaska. XPilot. http://www.xpilot.org//

[31] Hyun-Duk Cho, Kisuk Chung, and Taehoon Kim. Benefits of the big.LITTLE Architecture. In Technical Report, Samsung Electronics Co., Ltd., 2012.

[32] Reinhard Wilhelm, Jakob Engblom, Andreas Ermedahl, Niklas Holsti, Stephan Thesing, David Whalley, Guillem Bernat, Christian Ferdinand, Reinhold Heckmann, Tulika Mitra, Frank Mueller, Isabelle Puaut, Peter Puschner, Jan Staschulat, and Per Stenström. The Worst-Case ExecutionTime Problem - Overview of Methods and Survey of Tools. ACM Transactions on Embedded Computing Systems, 2008.

[33] Michael Roitzsch, Stefan Wächtler, and Hermann Härtig. ATLAS: LookAhead Scheduling Using Workload Metrics. In Proceedings of the 19th RealTime and Embedded Technology and Applications Symposium, 2013.

[34] Jian Chen and Lizy K. John. Efficient Program Scheduling for Heterogeneous Multi-core Processors. In Proceedings of the 46th Annual Design Automation Conference, 2009.

[35] Sadagopan Srinivasan, Ravishankar Iyer, Li Zhao, and Ramesh Illikkal. HeteroScouts: Hardware Assist for OS Scheduling in Heterogeneous CMPs. ACM SIGMETRICS Performance Evaluation Review, 39(1), 2011.

[36] Jason Cong and Bo Yuan. Energy-efficient Scheduling on Heterogeneous Multi-core Architectures. In Proceedings of the ACM/IEEE International Symposium on Low Power Electronics and Design, 2012.

[37] Kenzo Van Craeynest, Aamer Jaleel, Lieven Eeckhout, Paolo Narvaez, and Joel Emer. Scheduling Heterogeneous Multi-cores Through Performance Impact Estimation (PIE). In Proceedings of the 39th Annual International Symposium on Computer Architecture, 2012.

[38] Vinicius Petrucci, Michael A. Laurenzano, John Doherty, Yunqi Zhang, Daniel Mossé, Jason Mars, and Lingjia Tang. Octopus-Man: QoS-Driven Task Management for Heterogeneous Multicores in Warehouse-Scale Computers. In Proceedings of the 21st Symposium on High Performance Computer Architecture, 2015.

[39] E. Akyol and M. van der Schaar. Complexity Model Based Proactive Dynamic Voltage Scaling for Video Decoding Systems. Trans. Multi., 2007. 
[40] Steven Cameron Woo, Moriyoshi Ohara, Evan Torrie, Jaswinder Pal Singh, and Anoop Gupta. The SPLASH-2 Programs: Characterization and Methodological Considerations. In Proceedings of the 22Nd Annual International Symposium on Computer Architecture, 1995. 